\title{
The First Year of S-CUBED: The Swift Small Magellanic Cloud Survey
}

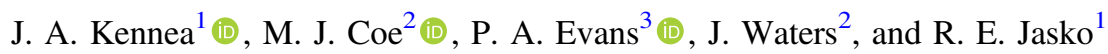 \\ ${ }^{1}$ Department of Astronomy and Astrophysics, The Pennsylvania State University, University Park, PA 16802, USA; jak51@psu.edu \\ ${ }^{2}$ Physics and Astronomy, University of Southampton, Southampton SO17 1BJ, UK \\ ${ }^{3}$ University of Leicester, X-ray and Observational Astronomy Research Group, Leicester Institute for Space and Earth Observation, \\ Department of Physics \& Astronomy, University Road, Leicester LE1 7RH, UK \\ Received 2018 March 21; revised 2018 October 11; accepted 2018 October 11; published 2018 November 19
}

\begin{abstract}
The Swift Small Magellanic Cloud (SMC) Survey, S-CUBED, is a high-cadence shallow X-ray survey of the SMC. The survey consists of 142 tiled pointings covering the optical extent of the SMC, which is performed weekly by NASA's Neil Gehrels Swift Observatory, with an exposure per tile of $60 \mathrm{~s}$. The survey is focused on discovery and monitoring of X-ray outbursts from the large known and unknown population of Be/X-ray binaries (BeXRBs) in the SMC. Given the very low background of Swift's X-ray telescope, even with a short exposure per tile, S-CUBED is typically sensitive to outbursts in the SMC at $>1 \%-2 \%$ Eddington luminosity for a typical $1.4 M_{\odot}$ neutron star compact object. This sensitivity, combined with the high cadence and the fact that the survey can be performed all year round, makes it a powerful discovery tool for outbursting accreting X-ray pulsars in the SMC. In this paper we describe results from the first year of observations of S-CUBED, which includes the 1SCUBEDX catalog of 265 X-ray sources, 160 of which are not identified with any previously cataloged X-ray source. We report on bulk properties of sources in the 1SCUBEDX catalog. Finally, we focus on results of S-CUBED observations of several interesting sources, which includes discovery of three Type II outbursts from BeXRBs and the detection of Type I outbursts and orbital periods in six BeXRB systems.
\end{abstract}

Key words: catalogs - surveys - X-rays: binaries

\section{Introduction}

The Small Magellanic Cloud (SMC) is an irregular dwarf galaxy located at a distance of approximately $62 \mathrm{kpc}$ (e.g., Haschke et al. 2012). In X-rays the SMC is extremely active, with the majority of the X-ray sources being high-mass X-ray binaries (HMXBs; see, e.g., Coe \& Kirk 2015). Many of these HMXB systems are X-ray pulsars (Yang et al. 2017), and approximately 98\% are known to have Be-star companions (Coe et al. 2005).

$\mathrm{Be} / \mathrm{X}$-ray binaries (BeXRBs) are HMXBs, typically containing a neutron star (NS) compact object, as evidenced by the detection of X-ray pulsations in many of these objects. BeXRBs with black hole compact objects have also been proposed, but so far only one system has been identified, MWC 656 (Casares et al. 2014). In BeXRBs the companion star is a massive B-type star, showing Balmer lines in emission, which leads to the Be-star classification. The emission lines arise from the presence of a circumstellar disk around the star, and it is the material in this disk that provides the fuel for accretion onto the compact object and hence gives rise to its X-ray emission.

BeXRBs show variability across a broad range of timescales and wavelengths. In X-ray, the variability of BeXRBs is often characterized as Type I and Type II outbursts. Type I outbursts occur regularly and are thought to be caused by the periastron passage of the NS passing close to the outer edge of the circumstellar disk. The regularity of Type I bursts is therefore linked to the orbital period of the system. Type II outbursts are much brighter, getting close to Eddington, and sometimes super-Eddington, luminosities (Townsend et al. 2017); these outbursts can last for several orbital periods and arise when the mass ejection from the Be star promotes an exceptionally large disk, often filling the entire orbit of the compact object.

The SMC has an overabundance of known BeXRB systems compared to the Milky Way owing to recent high periods of star formation (Harris \& Zaritsky 2004). It also benefits observationally from a low foreground extinction $\left(N_{\mathrm{H}}=\right.$ $5.34 \times 10^{20} \mathrm{~cm}^{-2}$; Willingale et al. 2013), and SMC X-ray sources have a relatively well constrained distance compared to those in the Milky Way. All of these factors make estimating the X-ray luminosity more accurate than for Milky Way objects. Consequently, the SMC provides an ideal laboratory for the discovery and study of BeXRBs in outburst. In this paper, we follow the naming system of Coe et al. (2005), where BeXRBs are named "SXP" (for SMC X-ray pulsar) followed by the pulsar period in seconds, e.g., SXP 91.1.

Previous observations of the SMC have been mostly focused on deep observations of the SMC, e.g., those surveys by ROSAT (Kahabka \& Pietsch 1996; Haberl et al. 2000), XMM-Newton (Sturm et al. 2013), and Chandra (Schurch et al. 2007; McGowan et al. 2008; Antoniou et al. 2009; Hong et al. 2017). However, an irregular, approximately weekly survey of selected regions of the SMC was performed over a period of more than a decade (Galache et al. 2008) by the Proportional Counter Array (PCA) on the Rossi $X$-ray Timing Explorer (RXTE; Bradt et al. 1993). RXTE was deactivated in 2012 January, ending those observations. Without regular sensitive monitoring observations of the SMC, discovery of transient outbursts was limited to those transients bright enough to be detected either by all-sky survey instruments, such as Swift's Burst Alert Telescope (BAT; Barthelmy et al. 2005), Fermi's Gamma-Ray Burst Monitor (Meegan et al. 2009), and the "Monitor of the All-sky X-ray Image" (MAXI; Matsuoka et al. 2009), or through infrequent scans of the SMC performed by INTEGRAL (Coe et al. 2010). In all these cases sensitivities of the telescopes involved were such that they were only capable of detecting the few extremely bright Type II outbursts.

NASA's Neil Gehrels Swift Observatory (Swift; Gehrels et al. 2004) is a Medium-Class Explorer (MIDEX) satellite, launched in 2004 November, with the primary goal of studying gamma-ray bursts (GRBs). Swift consists of three co-aligned 
instruments: BAT, which operates in the $15-150 \mathrm{keV}$ energy range with a 1.4 sr field of view (FOV); the X-ray Telescope (XRT; Burrows et al. 2005), with a 23!6 FOV operating in the 0.3-10 keV band; and the Ultra-violet/Optical Telescope (UVOT; Roming et al. 2005), with a 17' FOV, observing at wavelengths between 170 and $650 \mathrm{~nm}$.

The Swift SMC Survey (hereafter S-CUBED) was designed to harness the unique capabilities of Swift: its rapid slewing, which allows for low-overhead observing with very short exposure times, and its sensitive low-background XRT, to perform weekly X-ray observations of the SMC, in order to both hunt for outbursting BeXRB sources and monitor the flux of transient and persistent sources.

Although the SMC is relatively compact, the XRT's FOV only covers $\sim 0.12 \mathrm{deg}^{2}$; therefore, covering a significant fraction of the SMC requires many pointings. To achieve this, we utilized a new short-exposure tiling mode, developed to enable Swift to search large-area (tens to hundreds of square degrees) error regions associated with gravitational-wave detections by the Advanced LIGO and Advanced Virgo detectors (e.g., Evans et al. 2016b). Although this observation mode requires that each exposure is short $(60 \mathrm{~s})$ to cover this large area, S-CUBED is sensitive to outbursts from SMC sources at a level of $>3.5 \times 10^{36} \mathrm{erg} \mathrm{s}^{-1}$, or $>2 \%$ Eddington, for a typical BeXRB spectrum.

In this paper we describe the design, implementation, and results from S-CUBED, covering the first year of observations, which for the purposes of this paper includes all S-CUBED observations that were taken between 2016 June 8 and 2017 June 6. In addition, where follow-up Target of Opportunity (ToO) observations triggered by S-CUBED were taken by Swift or other observatories, we report on results of those observations, if not reported elsewhere.

This paper is novel in three ways. First, it describes a new method of performing X-ray surveys of large regions of the sky. S-CUBED represents the test bed of a new Swift tiled survey observing mode, which would not have been possible with other focused XRTs, primarily as it is powered by Swift's fast slewing capability, which allows for low-overhead shortexposure observations. Although regular scans of large regions such as the SMC have been performed by survey telescopes, or in scanning observations by missions such as $R X T E$, these surveys do not have the high sensitivity that S-CUBED has, nor do they have the subute-level spatial resolution required to accurately determine the source in outburst by localization.

Second, we report, for the first time, the detection of large Type II outbursts of BeXRBs in the SMC, SXP 6.85 and SXP 59.0. In addition, S-CUBED observations were the first to identify the major super-Eddington outburst of SMC X-3, which has been extensively reported on elsewhere (e.g., Townsend et al. 2017; Tsygankov et al. 2017; Weng et al. 2017; Koliopanos $\&$ Vasilopoulos 2018), and we present here S-CUBED focused results. These detections, along with detections of Type I outbursts, show the power of the S-CUBED observing technique in monitoring the large population of BeXRBs in the SMC, including outbursts that would be below the typical sensitivity of larger-area X-ray survey telescopes.

Third, we present a catalog of all high-quality detections of X-ray point sources in the SMC. Although the S-CUBED combined first-year data have a relatively shallow exposure time and lower sensitivity compared to previous X-ray surveys, such as those performed by XMM-Newton, Chandra, and
ROSAT, it covers both a larger spatial area and longer time period than those surveys, leading to the detection of 160 previously unknown X-ray sources, the details of which are reported for the first time in this paper.

\section{Survey Design}

The goals of S-CUBED are threefold. The first is to provide full coverage of the known X-ray sources that are located in the optical extent of the SMC, including the region known as the "Wing." The decision to limit to the optical core of the SMC was made in order to limit the exposure time required to perform the survey, but also to avoid strongly biasing the survey toward observing regions that have been previously well studied in $\mathrm{X}$-rays. The second is to perform the search at a sufficient level of sensitivity that a transient can be detected earlier than previously possible with less sensitive all-sky monitor telescopes such as BAT and MAXI. The third is to perform observations at a cadence sufficient for rapid reporting and good sampling of light curves of typical Type II outbursts of BeXRBs. All of these goals need to be met in a way that is acceptable to the operation of Swift and must not overburden the Swift observing schedule to the detriment of other observing programs.

In order to determine the area to tile, we utilized the X-ray source catalogs of ROSAT (Haberl et al. 2000) and XMMNewton (Sturm et al. 2013). The tiling pattern was generated algorithmically, by searching over a region $6^{\circ} \times 6^{\circ}$ centered on the SMC and ensuring that a tile was placed within any region within two XRT FOV radii (23!6) of regions where the source density was greater than three sources per field. No attempt to cover the LMC-SMC bridge was made, as this region covers too large an area.

XRT has an approximately circular FOV with an 11!8 radius. Swift's slewing is optimized for speed, which leads to a pointing error of up to $3^{\prime}$ (i.e., Swift can land up to $3^{\prime}$ off target). To compensate for this, the overlap between tiles was set to $3^{\prime}$, as this ensures gapless coverage. The survey is purposely conservative in its approach to covering the SMC, in order to ensure that we are not biasing the survey to just observing previously known sources, but also those in the gaps between them; therefore, the tiling is continuous.

The resulting configuration of the survey is shown in Figure 1. S-CUBED consists of 142 Swift pointings, covering an area of approximately 13.2 square degrees.

The XRT background is exceptionally low, the on-orbit instrumental background of Swift has been measured to be $10^{-6}$ counts s $^{-1}$ pixel $^{-1}$ (Evans et al. 2014), and XRT's pixel scale is $2.36 \mathrm{arcsec} / \mathrm{pixel}$. This means that in a typical circular XRT source extraction region with a 20 pixel radius, the background would be $\sim 1$ count in $1 \mathrm{ks}$. Assuming that the minimum required number of counts required for an XRT detection is 5 counts, as well as typical spectral parameters for a $\mathrm{BeXRB}$ in the SMC, for a $60 \mathrm{~s}$ exposure we would detect any source brighter than a luminosity of $2 \%$ of $L_{\mathrm{Edd}}$. For a softer spectrum source (e.g., a Crab-like spectrum), this sensitivity is increased to $1 \%$ of $L_{\text {Edd }}$. Given the typical outburst brightness of BeXRBs, which can reach super-Eddington levels (e.g., SMC X-3; Townsend et al. 2017), it was determined that utilizing the minimum exposure time allowed would give sufficient sensitivity, as well as minimizing the load on the Swift schedule.

For cadence, typical orbital periods of BeXRBs are of the order of 10-100 days, and outburst rise times typically scale to 


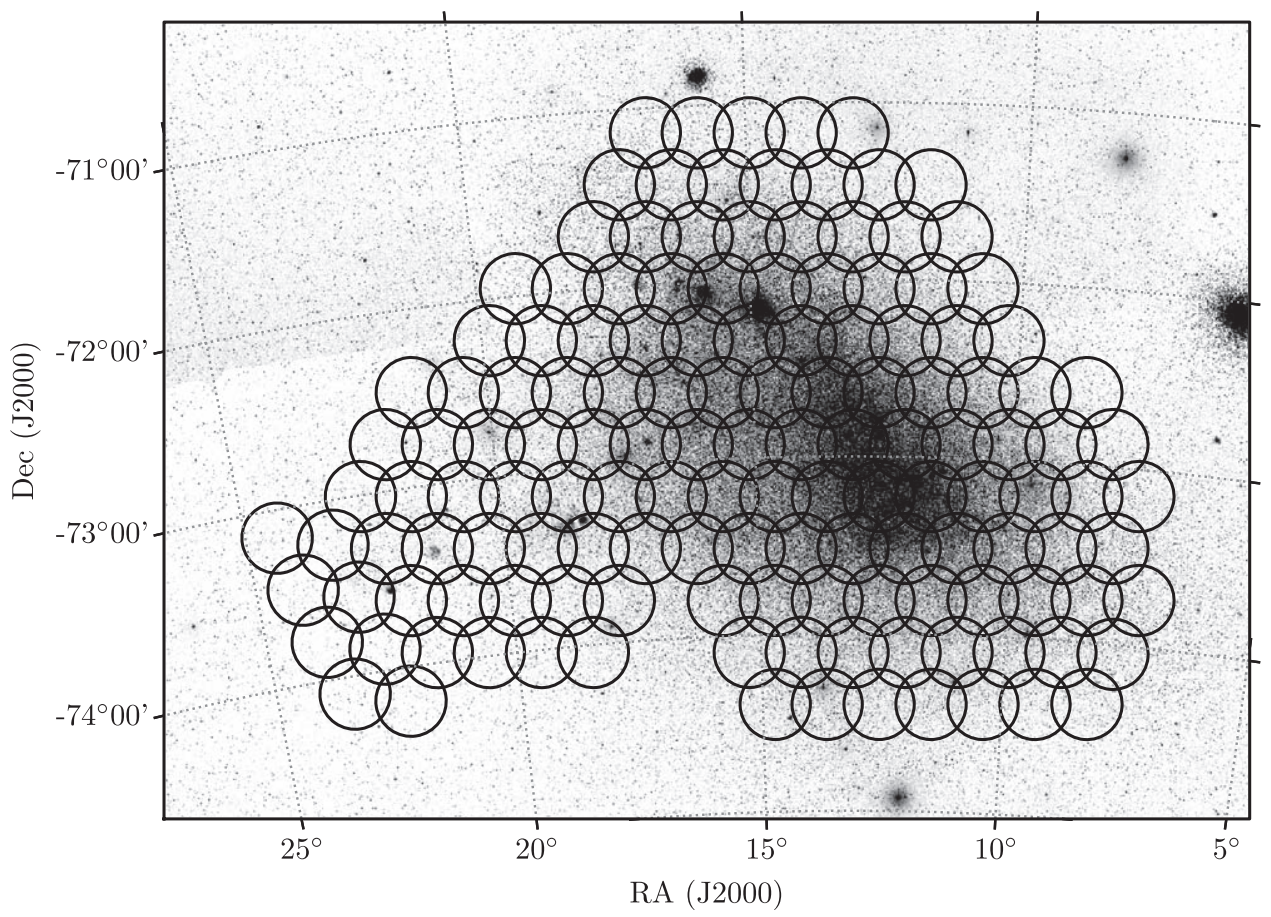

Figure 1. Configuration of the S-CUBED survey. The survey consists of 142 tiles covering the central optical extent of the SMC and Wing. Overlaid tiles are from the Digitized Sky Survey.

be approximately on the order of one orbital period. Due to limitations of onboard memory, normal tiling can only be performed when single-day length plans are uploaded to Swift (Tuesday to Thursday), so the Swift Flight Operations Team preferred that the S-CUBED survey be scheduled at intervals of a fixed number of weeks, to ensure that the observations occurred on the same day of the week. Therefore, in order to maximize our coverage of outbursts, and to ensure the quickest response time to new outbursting sources, it was decided to schedule S-CUBED at a cadence of once per week (see next section).

\section{Observations}

S-CUBED observations were performed with the aim of observing 142 tiles covering the SMC every week, with an individual exposure per tile of $60 \mathrm{~s}$, to a total of $8.52 \mathrm{ks}$, not including slewing overheads. Due to the short distance between the tiles in the survey, the median slewing time between tiles was $23 \mathrm{~s}$. The Swift scheduling system cannot handle observations shorter than 5 minutes, and schedules targets at a $60 \mathrm{~s}$ time resolution, which would be inefficient for scheduling $60 \mathrm{~s}$ exposure tiles. Therefore, a custom planning solution was developed where S-CUBED tiles are scheduled as a single pointing near the center of the survey and then replaced with tiles, using high-accuracy estimates for slewing and visibility.

Due to observing constraints, higher-priority targets, or interruption by ToO and GRB observations, S-CUBED was not always observed to $100 \%$ completion every week. Swift's orbit pole constraint, which typically lasts up to 10 days, was the primary cause of S-CUBED observations not being scheduled.

In the first year, S-CUBED observations were performed on 43 out of 52 weeks, and the average completion rate for weeks when S-CUBED tiling was performed was $95 \%$ of all tiles. Details of the individual tiling observations performed for S-CUBED are given in Table 1. Note that observations in the first 5 weeks of S-CUBED were attempted at a cadence of every 8 days, in order to reduce UVOT filter wheel rotations by picking days on which the filter of the day was $u v w 1$; however, due to the limitation that the survey could not be performed on Friday-Monday, this was changed to a 7-day cadence, in order to perform the survey on Tuesdays. In some cases where the S-CUBED survey was not completed in a single day, observations were performed on the following days in order to make up lost time. An image of the exposure map of S-CUBED is shown in Figure 2. The median exposure of a non-overlapping region of the S-CUBED survey was $1919 \mathrm{~s}$.

As it is often hard to predict the amount of slew time required between tiles, sometimes more time was scheduled than was required to observe the 142 S-CUBED tiles. In those cases additional time was spent on a field that centered on the SMC, which can be clearly seen in Figure 2 as a region of enhanced exposure.

When, as the result of S-CUBED observations, an outburst of an X-ray transient source in the SMC is found, follow-up additional observations were requested through $\mathrm{ToO}$ requests to Swift or other telescopes such as NuSTAR and Chandra as appropriate, in order to obtain higher-quality spectral and timing information. We report on the results from those observations along with S-CUBED data in Section 6.

\section{Data Analysis}

Analysis of large tiled regions is computationally intensive, and combining the entire S-CUBED surveyed region proved to be too slow and memory intensive. However, analysis of individual tiles creates a different problem: how to deal with the fact that tiles overlap and many sources will appear in more than one tile. To achieve maximum sensitivity, it is best to perform some combining of tiles, but the number of tiles cannot be so large as to make the analysis computationally slow, as we are aiming for near-real-time reporting of transients. 
Table 1

Observations Performed by Swift for the S-CUBED Program in the First Year of the Program

\begin{tabular}{|c|c|c|c|c|}
\hline Week & Start Time (UTC) & End Time (UTC) & Tiles Observed & Exposure \\
\hline 1 & 2016 Jun 08 00:06:02 & 2016 Jun 08 00:06:57 & $142(100 \%)$ & $8.5 \mathrm{ks}$ \\
\hline 2 & 2016 Jun 16 00:06:02 & 2016 Jun 16 01:06:11 & $78(55 \%)$ & $4.6 \mathrm{ks}$ \\
\hline $3^{\mathrm{a}}$ & 2016 Jun 24 00:06:02 & 2016 Jun 24 00:06:52 & $127(89 \%)$ & $7.5 \mathrm{ks}$ \\
\hline $4^{\mathrm{a}}$ & 2016 Jun 28 01:06:02 & 2016 Jun 28 01:06:34 & $142(100 \%)$ & $8.9 \mathrm{ks}$ \\
\hline $5^{\mathrm{a}}$ & 2016 Jul 06 02:07:01 & 2016 Jul 06 02:07:52 & $142(100 \%)$ & $8.8 \mathrm{ks}$ \\
\hline $5^{\mathrm{a}}$ & 2016 Jul 10 08:07:02 & 2016 Jul 10 08:07:59 & $142(100 \%)$ & $8.7 \mathrm{ks}$ \\
\hline 6 & 2016 Jul 15 06:07:02 & 2016 Jul 15 06:07:55 & $142(100 \%)$ & $8.8 \mathrm{ks}$ \\
\hline 8 & 2016 Jul 29 04:07:02 & 2016 Jul 29 04:07:42 & $139(98 \%)$ & $8.6 \mathrm{ks}$ \\
\hline 9 & 2016 Aug 03 00:08:02 & 2016 Aug 03 00:08:28 & $139(98 \%)$ & $8.9 \mathrm{ks}$ \\
\hline 10 & 2016 Aug 10 00:08:02 & 2016 Aug 10 00:08:34 & $142(100 \%)$ & $8.9 \mathrm{ks}$ \\
\hline 11 & 2016 Aug 17 01:08:58 & 2016 Aug 17 01:08:06 & $138(97 \%)$ & $8.2 \mathrm{ks}$ \\
\hline 12 & 2016 Aug 24 06:08:02 & 2016 Aug 24 06:08:39 & $142(100 \%)$ & $8.8 \mathrm{ks}$ \\
\hline 13 & 2016 Aug 31 01:08:02 & 2016 Aug 31 01:08:32 & $142(100 \%)$ & $8.9 \mathrm{ks}$ \\
\hline 16 & 2016 Sep 21 00:09:02 & 2016 Sep 21 00:09:02 & $142(100 \%)$ & $8.9 \mathrm{ks}$ \\
\hline 17 & 2016 Sep 28 04:09:02 & 2016 Sep 28 04:09:37 & $99(70 \%)$ & $6.0 \mathrm{ks}$ \\
\hline 18 & 2016 Oct 05 08:10:02 & 2016 Oct 05 08:10:27 & $142(100 \%)$ & $8.7 \mathrm{ks}$ \\
\hline 19 & 2016 Oct 12 05:10:02 & 2016 Oct 12 06:10:51 & $142(100 \%)$ & $8.6 \mathrm{ks}$ \\
\hline 20 & 2016 Oct 19 07:10:02 & 2016 Oct 19 07:10:31 & $137(96 \%)$ & $8.5 \mathrm{ks}$ \\
\hline 21 & 2016 Oct 25 06:10:02 & 2016 Oct 25 06:10:28 & $145(100 \%)$ & $9.3 \mathrm{ks}$ \\
\hline 23 & 2016 Nov 09 09:11:02 & 2016 Nov 09 09:11:03 & $142(100 \%)$ & $8.7 \mathrm{ks}$ \\
\hline 24 & 2016 Nov 16 00:11:02 & 2016 Nov 16 00:11:34 & $142(100 \%)$ & $8.7 \mathrm{ks}$ \\
\hline 25 & 2016 Nov 23 13:11:02 & 2016 Nov 23 13:11:33 & $139(98 \%)$ & $8.3 \mathrm{ks}$ \\
\hline 26 & 2016 Nov 30 00:11:02 & 2016 Nov 30 00:11:21 & $142(100 \%)$ & $8.5 \mathrm{ks}$ \\
\hline 27 & 2016 Dec 07 04:12:02 & 2016 Dec 07 04:12:09 & $142(100 \%)$ & $8.9 \mathrm{ks}$ \\
\hline 28 & 2016 Dec 14 00:12:02 & 2016 Dec 14 00:12:57 & $140(99 \%)$ & $8.7 \mathrm{ks}$ \\
\hline 30 & 2016 Dec 28 06:12:02 & 2016 Dec 28 06:12:56 & $142(100 \%)$ & $8.5 \mathrm{ks}$ \\
\hline 31 & 2017 Jan 05 12:01:02 & 2017 Jan 05 12:01:01 & $142(100 \%)$ & $8.8 \mathrm{ks}$ \\
\hline 32 & 2017 Jan 11 02:01:02 & 2017 Jan 11 02:01:33 & $142(100 \%)$ & $9.5 \mathrm{ks}$ \\
\hline 33 & 2017 Jan 18 01:01:02 & 2017 Jan 18 01:01:10 & $107(75 \%)$ & $6.5 \mathrm{ks}$ \\
\hline 34 & 2017 Jan 25 00:01:02 & 2017 Jan 25 00:01:12 & $142(100 \%)$ & $8.5 \mathrm{ks}$ \\
\hline 35 & 2017 Feb 01 00:02:02 & 2017 Feb 01 00:02:31 & $142(100 \%)$ & $8.7 \mathrm{ks}$ \\
\hline 38 & $2017 \mathrm{Feb} 22$ 00:02:02 & 2017 Feb 22 00:02:02 & $142(100 \%)$ & $8.4 \mathrm{ks}$ \\
\hline 40 & 2017 Mar 08 00:03:02 & 2017 Mar 08 00:03:57 & $122(86 \%)$ & $7.3 \mathrm{ks}$ \\
\hline 41 & 2017 Mar 14 02:03:02 & 2017 Mar 14 02:03:26 & $140(99 \%)$ & $8.6 \mathrm{ks}$ \\
\hline 42 & 2017 Mar 22 00:03:02 & 2017 Mar 22 00:03:31 & $117(82 \%)$ & $7.2 \mathrm{ks}$ \\
\hline 43 & 2017 Mar 29 12:03:02 & 2017 Mar 29 13:03:20 & $136(96 \%)$ & $8.7 \mathrm{ks}$ \\
\hline 45 & 2017 Apr 11 01:04:02 & 2017 Apr 11 01:04:37 & $126(89 \%)$ & $8.1 \mathrm{ks}$ \\
\hline 46 & 2017 Apr 18 00:04:02 & 2017 Apr 18 00:04:07 & $142(100 \%)$ & $8.7 \mathrm{ks}$ \\
\hline 47 & 2017 Apr 25 00:04:02 & 2017 Apr 25 00:04:38 & $116(82 \%)$ & $6.9 \mathrm{ks}$ \\
\hline 48 & 2017 May 02 00:05:02 & 2017 May 02 00:05:23 & $140(99 \%)$ & $8.6 \mathrm{ks}$ \\
\hline 49 & 2017 May 09 00:05:02 & 2017 May 09 00:05:32 & $140(99 \%)$ & $8.5 \mathrm{ks}$ \\
\hline 50 & 2017 May 16 01:05:02 & 2017 May 16 01:05:38 & $129(91 \%)$ & $8.2 \mathrm{ks}$ \\
\hline 53 & 2017 Jun 06 00:06:02 & 2017 Jun 06 00:06:05 & $117(82 \%)$ & $7.2 \mathrm{ks}$ \\
\hline
\end{tabular}

Notes. In total 43 S-CUBED tilings were performed during the first year of observations, which started on 2016 June 8 and ended on 2017 June 6 . Missing weeks are typically caused by the SMC being pole constrained (see text), or by the observations being not scheduled as a result of higher-priority observations.

${ }^{a}$ Note that in weeks 3, 4, and 5, S-CUBED observations were taken at a slightly higher cadence (every 4 days). This resulted in there being two sets of tiling observations performed in week 5 , and hence is why week 5 appears twice in the table.

Therefore, the individual XRT tiles were grouped into "blocks," which includes all tiles up to $\sim 0.6$ in radius from the center of the block (corresponding to three XRT fields in diameter). Blocks were defined such that every field and every overlap between fields was included in at least one block, while creating the minimum number of blocks necessary to ensure this. Analysis was then performed on a block-by-block basis as described below. Note that some fields are in multiple blocks, in which case the receipt of data triggered the analysis of multiple blocks; such analyses were performed independently.

To analyze a block, source detection was carried out using the iterative cell-detect algorithm of Evans et al. (2014), after every observation. This algorithm assigns each source a quality flag of "Good," "Reasonable," or "Poor," which relates to how likely it is to be a real astrophysical source. These adjectival ratings are defined so that for "Good" sources we expect a false-positive rate of $0.3 \%$, for "Reasonable" a false-positive rate of $1 \%$, and for "Poor" sources a false-positive rate of $10 \%$. These false-positive rates were calculated utilizing extensive simulations as described by Evans et al. (2014) for the 1SXPS catalog, with the strict definitions of those flags listed in Table 11 of that paper. As the pipeline for detection used in S-CUBED is derived from that used by the 1SXPS catalog, the false-positive rate will be identical to those calculated for 1SXPS.

This analysis was performed on the sum of all data collected for that block at the time of analysis, i.e., we did not search for sources in each weekly $60 \mathrm{~s}$ data set, but only in the summed 


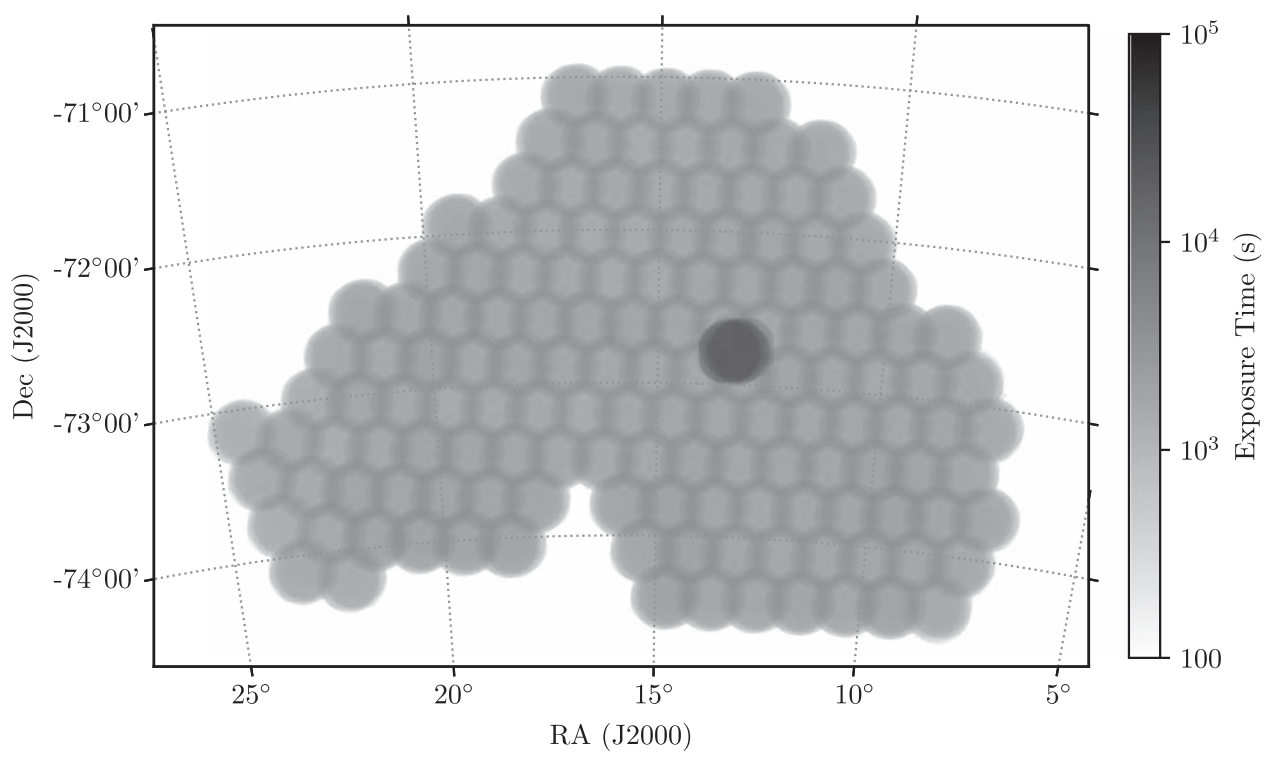

Figure 2. Exposure map of the S-CUBED survey for the first year of observations. Individual ToO observations of S-CUBED triggered targets are not included in this map, as S-CUBED data only process PC mode data, and ToO observations are typically in WT to allow accurate measurement of the pulsar period. Note that the region of higher exposure is due to overscheduling of observations, which means that after all tiles are scheduled some time is spent observing a field at the approximate center of the SMC to fill the remaining time.

data set of all S-CUBED observations taken so far. Therefore, as the S-CUBED cumulative exposure built up, the analysis system is able to detect sources that are fainter, building up a large catalog of X-ray sources. All objects detected in analysis of each block were compared with the list of sources already detected in the S-CUBED survey; sources whose position agreed to within $5 \sigma$ were identified with that source. If a source does not match a previously detected one, then it is added to the source list. Such new sources can potentially be either the result of the source brightening (i.e., a new transient) or simply the detection of a faint source made possible by the longer exposure.

This step of merging sources could only be called by one block at a time to prevent a newly detectable source being added to the source list multiple times. For sources that are detected multiple times during the survey, the best position of the source was defined as that detection that yielded the best quality flag (i.e., the position from a "Good" detection supersedes a "Reasonable" detection). If multiple detections had the same quality flag, then the position was taken from the detection with the highest signal-to-noise ratio $(\mathrm{S} / \mathrm{N})$, and if there were multiple detections with the same quality flag and $\mathrm{S} / \mathrm{N}$, then that with the smallest position uncertainty was taken.

A list of all sources detected in the block, along with any preexisting sources that lay within the block but were not detected in the latest analysis, was then produced. These sources were then each analyzed individually. The pipeline keeps track of which data were used in each source analysis; therefore, if an observation of a given source was part of two blocks, the source analysis was only conducted once.

For each source we produced a light curve, spectrum, and, where possible, an "enhanced" position (utilizing UVOT to reduce the systematic error on astrometry), using the tools described by Goad et al. (2007) and Evans et al. (2007, 2009), which corrected for vignetting, dead columns on the CCD, pileup, and other effects. Note that these tools are designed and calibrated for point sources only: the fluxes for extended sources such as the supernova remnant (SNR) 1E 0102.2
-7219 are not reliable. By default light curves were produced to have one bin per observation to give an at-a-glance idea of variability. However, for sources fainter than $\sim 0.1$ count $\mathrm{s}^{-1}$ such a light curve will be composed entirely of upper limits because although the source was solidly detected in the summed data set, no single $60 \mathrm{~s}$ observation is enough to yield a flux measurement that is nonzero at the $3 \sigma$ level. The automated analysis was made available to the S-CUBED team via a web interface, which allows easy rebinning of the light curve, allowing us to manually investigate the variability of such faint sources.

Due to the large number of sources detected, it is impractical to manually examine each source for signs of outbursting activity. Instead, the analysis software automatically identifies sources that may be transient or in outburst. For known sources, this is done by comparing the peak flux in the S-CUBED light curve with the cataloged flux; for previously unknown sources the peak S-CUBED flux is compared with a $3 \sigma$ upper limit calculated from the ROSAT All-Sky Survey (Voges et al. 1999) at the location of the source. In either case, if the S-CUBED flux is at least $5 \sigma$ above the comparison flux, the source is flagged.

For any source thus flagged, the pipeline sends a notification to the S-CUBED team members (keeping track of such notifications to avoid repeated alerts for a single outburst), which then allows for the light curve to be checked by hand. Since this check is performed on the per-observation light curve, it implicitly requires a source to be bright enough to produce a detection in the light curve $\left(\sim 0.1\right.$ counts $\left.\mathrm{s}^{-1}\right)$ before it can be classed as in outburst; thus, it is possible that there are objects in our sample that have undergone outbursts at a lower level but are not identified by the real-time analysis software. Therefore, for this paper we reanalyzed all pipeline-generated light curves to look for signs of transient behavior in them.

As described in Section 3, in some cases we obtained additional observations of such sources with Swift to monitor the outburst. Such observations were not included in the automated analysis or when performing source detection; in this case source products 


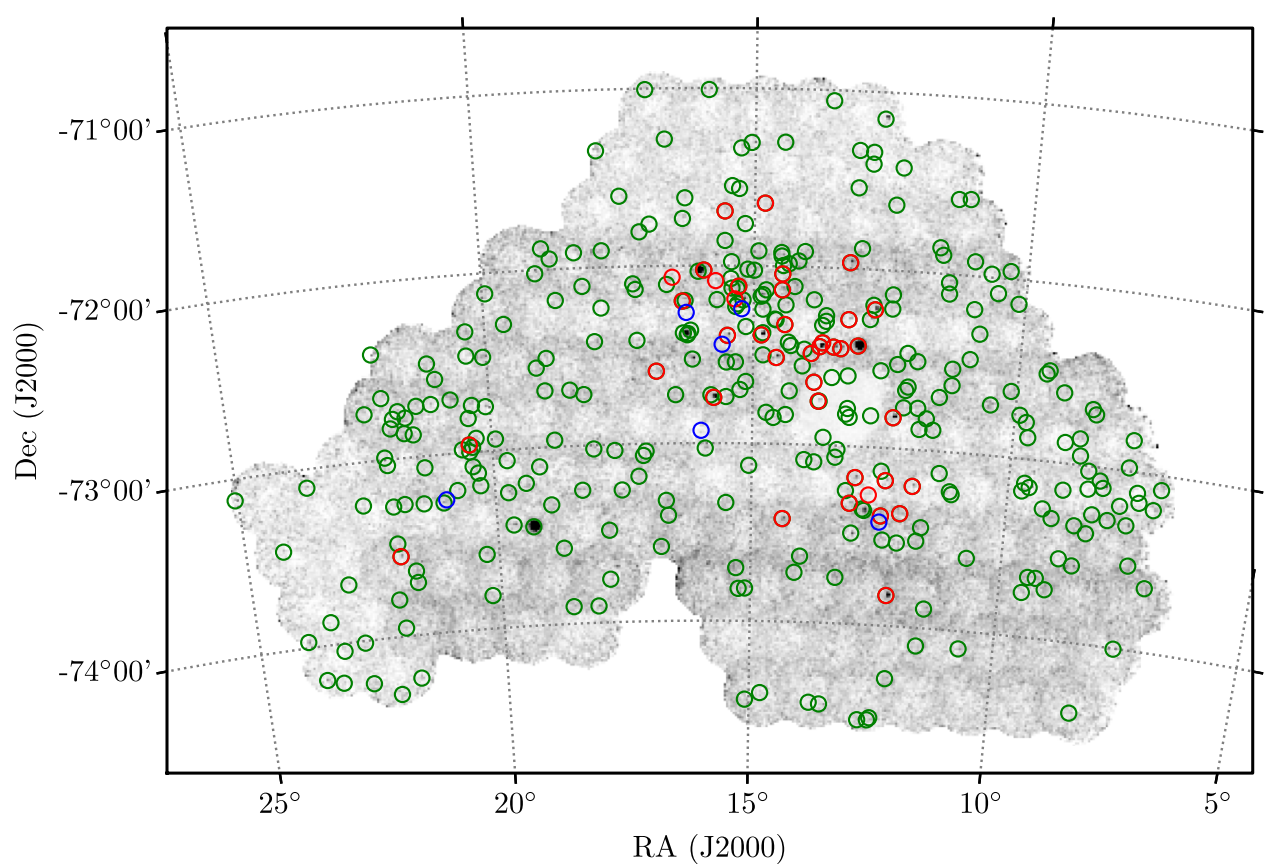

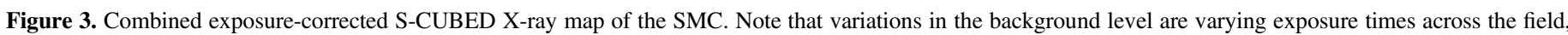

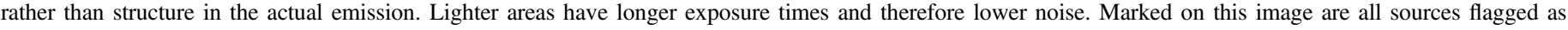
"Good" by S-CUBED: red circles are known "SXP" targets, blue circles are HMXBs identified in Table 3, and green circles are all others.

were built using the online tools at http://www.swift.ac.uk/user_ objects, described by Evans et al. (2009).

For results reported in this paper we assume a mean SMC distance of $62 \mathrm{kpc}$, and unless otherwise stated, all errors are $1 \sigma$, except for localization errors, which are given as a $90 \%$ confidence radius.

\section{1SCUBEDX: The First-year S-CUBED Source Catalog}

The combined S-CUBED X-ray image of the SMC, corrected for variations in exposure, is shown in Figure 3. The median exposure time over the whole mosaic is $1919 \mathrm{~s}$, although the survey contains regions of higher exposure owing to overlapping tiles and other overexposed regions as shown in Figure 2. Prominent in the field are several bright, well-known $\mathrm{X}$-ray sources, the brightest three of which are SMC X-3, which underwent an outburst in 2016-2017 (Townsend et al. 2017), SMC X-1, a persistent but highly variable HXMB (e.g., Li \& van den Heuvel 1997), and the X-ray-bright SNR 1E 0102.2-7219, which is most often utilized as an X-ray calibration source (Plucinsky et al. 2017). A large number of other point sources are visible in the image. Absent is any strong diffuse $\mathrm{X}$-ray emission, as expected owing to the relative insensitivity of the S-CUBED survey. The primary purpose of $\mathrm{S}$-CUBED is to examine variability of the X-ray point-source population in the SMC and to detect any turn-on of transient sources. However, in addition to this, S-CUBED also detected a great number of X-ray point sources that were previously unknown. In this section we present the results of detection, localization, and periodicity searches of point sources detected by S-CUBED, over the first year of observations, and present the first year catalog of S-CUBED sources: 1SCUBEDX.

Automated data analysis of the first year of S-CUBED observations as described in Section 4 detected a total of 806 point sources. Of these, 265 were flagged as "Good" and are therefore considered to have a high likelihood of being real X-ray point sources, 110 were "Reasonable," and 431 were "Poor." To limit the scope of the results presented in this paper, we only report on those sources that are flagged as "Good" by our data analysis. Based on the statistical simulations performed by Evans et al. (2014), the number of spurious sources flagged as "Good" is expected to be less than 1.

We present in Table 2 a catalog of all the "Good" sources detected in X-ray during the first year of S-CUBED observations. Hereafter we refer to this catalog as 1SCUBEDX, and sources in this catalog are named with the convention 1SCUBEDX JHHMMSS.s \pm DDMMSS, based on their S-CUBED-derived coordinates. In addition, as a shorthand for sources, we refer to targets by an internal catalog number, of the form "SCn," where $n$ corresponds to the order of discovery by the S-CUBED analysis software. Hence, the brightest source in the SMC, SMC X-1, is SC1, the bright SNR 1E 0102.2-7219 is SC2, and so on.

For sources where a spectral fit was possible, we quote a fitted photon index, assuming a standard SMC absorption. In addition, we calculate from this fit a counts-to-flux ratio and use this value to convert count rates in individual observations into fluxes. In cases where a spectral fit is not possible, we calculate the mean flux by taking the mean count rate and multiplying it by the median counts-to-flux ratio, derived from the average spectra of all point sources with a spectral fit, of $3 \times 10^{-11} \mathrm{erg} \mathrm{cm}^{-2}$ count $^{-1}$. The mean flux across all observations is given for each source.

In addition, for each source we give a detection percentage, which indicates how often the source was detected in individual S-CUBED tiling observations. In cases where this value was zero, this indicates that the source was not detected in any individual observation but was detected in the combined first year of observations. 


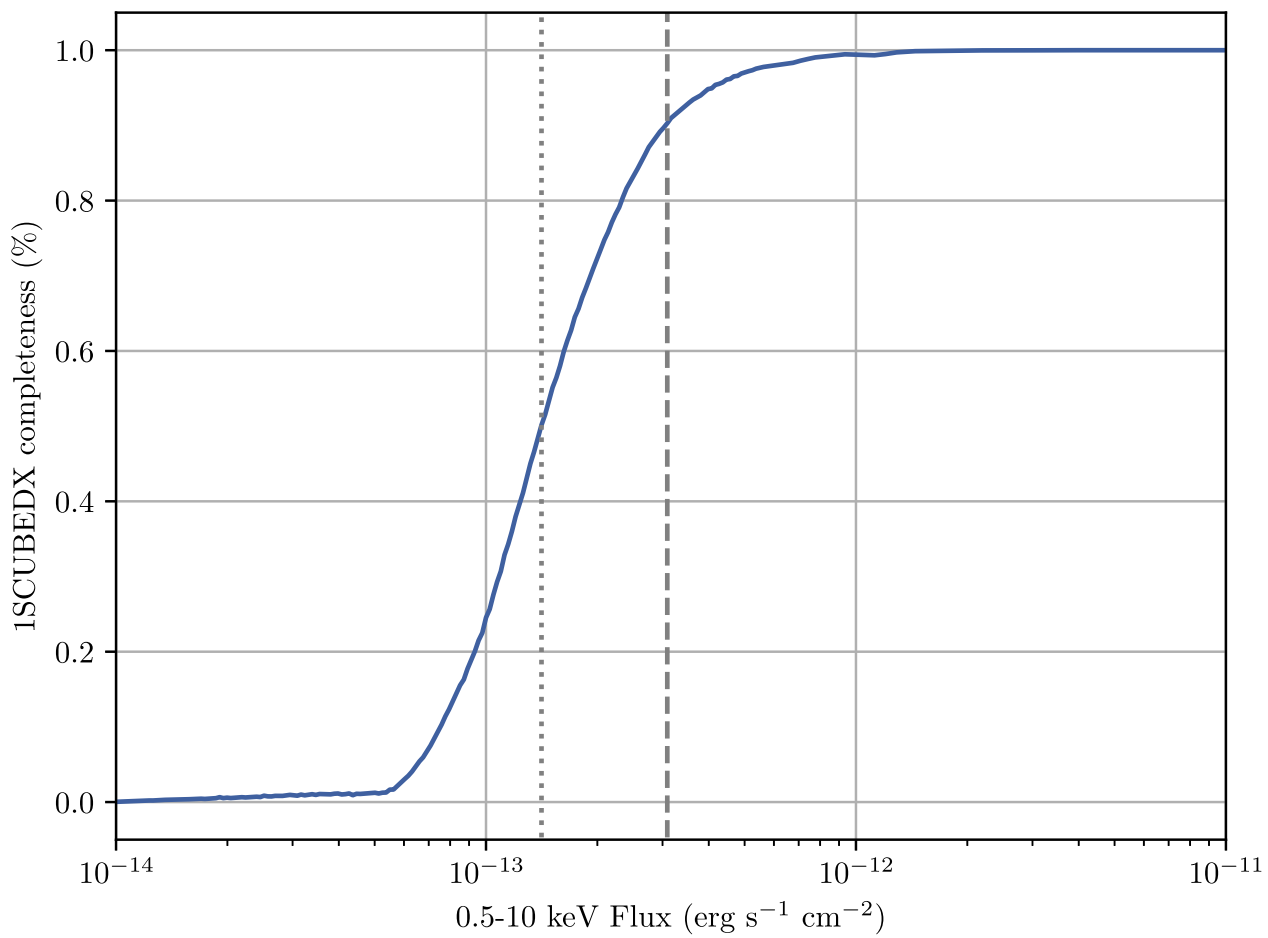

Figure 4. Simulated completeness function for the 1SCUBEDX survey. The $90 \%$ and 50\% completeness fluxes are marked as dashed and dotted lines, respectively.

Source positions were matched against X-ray source catalogs, including the 1SXPS (Evans et al. 2014), 3XMM-DR5 (Rosen et al. 2016), and HEASARC X-ray master catalogs, ${ }^{4}$ using positional coincidence. 1SCUBEDX sources are classified into two categories: known $(\mathrm{K})$ and unknown $(\mathrm{U})$, which specifically refers to whether they are previously identified X-ray emitters. In the 1SCUBEDX catalog, 105 sources are identified as known and 160 as unknown. Therefore, 1SCUBEDX represents a significant increase in the number of X-ray sources in the vicinity of the SMC, despite the relatively shallow overall exposure. In Table 2, for all known X-ray sources, we give a common catalog name for the X-ray source.

For X-ray sources where the identification of the X-ray source is known, for example, known BeXRBs and HMXBs as given by the catalogs of Coe \& Kirk (2015) and Haberl \& Sturm (2016), we list the common name and source type. In addition, we have cross-referenced the 1SCUBEDX catalog against several other catalogs of SMC sources, and where a positive match is made based on positional coincidence, the name of the source and the reported source type are given, along with references to which catalogs this source appears in. Based on this positional matching, we find 16 1SCUBEDX sources consistent with known active galactic nuclei (AGNs), 4 of which are detected in X-ray for the first time by S-CUBED.

Full investigation of source types for all S-CUBED X-ray sources is outside the scope of this paper and will be presented in a future work.

\subsection{SCUBEDX Completeness}

For any catalog, it is important to understand the expected completeness of the survey as a function of flux. As 1SCUBEDX was generated utilizing the same analysis methods as the 1SXPS catalog, we used the same calculations

\footnotetext{
4 https://heasarc.gsfc.nasa.gov/W3Browse/all/xray.html
}

for estimating completeness that were formulated for that catalog. Evans et al. (2014) describe in detail the simulation process utilized to obtain this completeness estimate, and Figure 14 within that paper shows the expected 50\% and 90\% completeness levels for sources classified as "Good" at a variety of exposures and flux levels. The median exposure time for the 1SCUBEDX catalog is 1919 s. Based on the calculations for 1SXPS, the expected flux at which the median exposure is $50 \%$ complete is $2 \times 10^{-13} \mathrm{erg} \mathrm{cm}^{-2} \mathrm{~s}^{-1}$, and it will be $90 \%$ complete above $4 \times 10^{-13} \mathrm{erg} \mathrm{cm}^{-2} \mathrm{~s}^{-1}$.

However, as the exposure time of S-CUBED is not uniform, simply using the median exposure time as an estimate of completeness would underestimate the occurrence of faint sources in regions of higher exposure (e.g., tile overlaps). Therefore, in order to better calculate the completeness of the entire 1SCUBEDX catalog, we performed a Monte Carlo simulation based on the configuration of S-CUBED observations taken in the first year.

In this simulation we estimated the completeness for 500 logarithmically spaced flux levels in the range of $10^{-14}-10^{-11} \mathrm{erg} \mathrm{cm}^{-2} \mathrm{~s}^{-1}$. For each flux level we simulated an X-ray source at random coordinates inside the survey and calculated the likelihood of its detection. By repeating this over 20,000 trials, we were able to estimate the completeness rate of the 1SCUBEDX survey at each flux level. This completeness rate is shown in Figure 4.

These simulations show that the 1SCUBEDX catalog is $>50 \%$ complete above a flux level of $1.4 \times 10^{-13} \mathrm{erg}$ $\mathrm{cm}^{-2} \mathrm{~s}^{-1}$ and $>90 \%$ complete above a flux level of $3.1 \times 10^{-13} \mathrm{erg} \mathrm{cm}^{-2} \mathrm{~s}^{-1}$.

\subsection{Luminosity Functions}

The distributions of the mean fluxes for known HMXBs (including SXPs and other identified HMXBs) and all other 1SCUBEDX sources are given in Figure 5. It is seen that the 


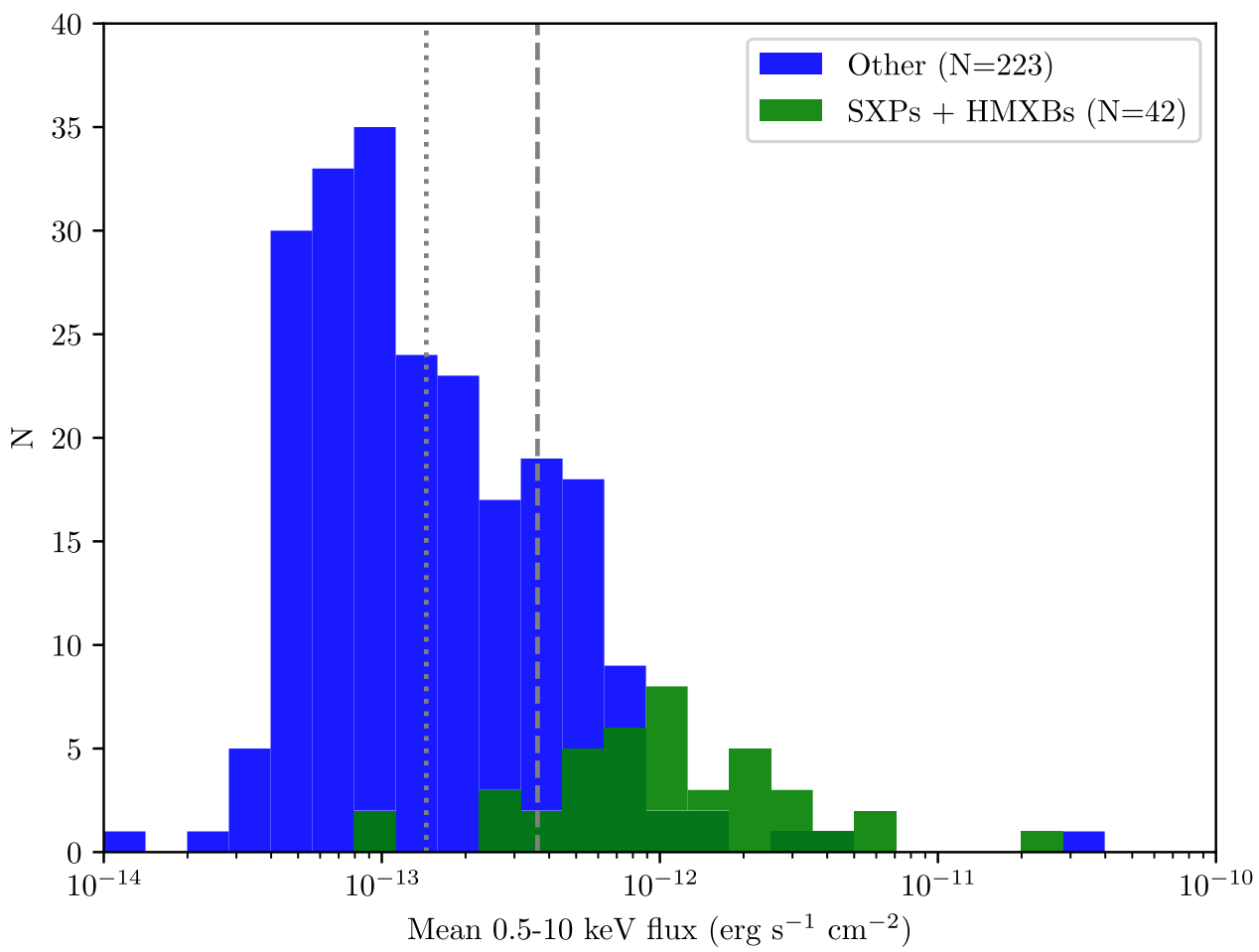

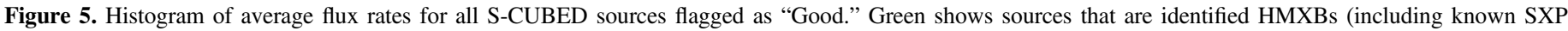

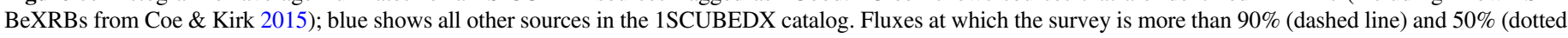
line) complete are shown.

average flux of the HMXB sources is typically higher than that of the other sources; the median of the average fluxes is $1.15 \times 10^{-12} \mathrm{erg} \mathrm{s}^{-1} \mathrm{~cm}^{-2}$ for SXP sources and $1.20 \times$ $10^{-13} \mathrm{erg} \mathrm{s}^{-1} \mathrm{~cm}^{-2}$ for the unidentified X-ray sources, almost an order of magnitude lower. We note that the majority of the unidentified sources are at mean fluxes below the $90 \%$ completeness, suggesting a large population of fainter sources.

To further compare the sources from S-CUBED with other characterizations of the HMXB population within the SMC, a cumulative histogram of the X-ray flux was plotted, and a subsequent luminosity function was computed using a leastsquares fit to the distribution.

The results are shown in Figure 6.

The least-squares fit to the HMXB distribution in Figure 6 gives a slope of $\alpha=-0.64 \pm 0.03$. Hence, the resulting luminosity function is given by Equation (1), where $N_{\mathrm{HMXB}}$ is the number of sources with a flux greater than $S$. $S$ is the $0.5-10 \mathrm{keV}$ flux in units of erg s $\mathrm{s}^{-1} \mathrm{~cm}^{-2}$ :

$$
N_{\text {HMXB }}=10^{-6.5 \pm 0.4} S^{-0.64 \pm 0.03} \text {. }
$$

Shtykovskiy \& Gilfanov (2005) quote the universal HMXB luminosity function, given by Grimm et al. (2003), to have a slope of $\alpha_{G}=-0.6$, which is in close agreement with the SCUBED-derived value.

A similar histogram was also plotted for the unidentified S-CUBED sources, for which we removed all sources for which a source type has been previously identified (mostly SNRs, AGNs, and foreground stars). This can be seen in Figure 6.

The slope for this distribution is seen to be steeper and has a slope of $\alpha=-0.97 \pm 0.06$. However, we note that the cumulative distribution shows significant flattening toward the faint end, likely the signature of the incompleteness of 1SCUBEDX at lower flux levels.

To compensate for this, we fitted the cumulative distribution function only for fluxes above $90 \%$ completeness, and we obtain a steeper $\alpha=1.57 \pm 0.07$. We also computed a cumulative distribution function for unknown sources corrected for the estimated catalog completion. We note that the fitted luminosity function for fluxes above $90 \%$ completion shows good agreement with the estimated distribution below the $90 \%$ completeness threshold.

Hence, the resulting luminosity function for sources of unknown type is given by Equation (2), where $N_{\text {Unidentified }}$ is the number of sources with a luminosity greater than $S$ :

$$
N_{\text {Unidentified }}=10^{-18.0 \pm 0.8} S^{-1.57 \pm 0.07} \text {. }
$$

Shtykovskiy \& Gilfanov (2005) quote results of their luminosity function parameters when all likely HMXB candidates are removed from the data set. According to their results, a slope of $\alpha=1.48 \pm 0.12$ was obtained when excluding only the likely candidates, and a slope of $\alpha=1.55 \pm 0.13$ was obtained when excluding all possible HMXB candidates. Given the value of the slope of unidentified S-CUBED sources quoted above of $\alpha=$ $1.57 \pm 0.07$, we find close agreement between the value for 1SCUBEDX unidentified sources and the quoted values from Shtykovskiy \& Gilfanov (2005). Our derived value for unidentified sources is also consistent with the expected value for a Euclidean slope $(\alpha=1.5)$, suggesting that the majority of the unidentified source population are likely background sources, such as AGNs.

\subsection{Duty Cycles}

The duty cycle (DC) of an HMXB is defined by the fraction of the time that they are in outburst. While physically this may 


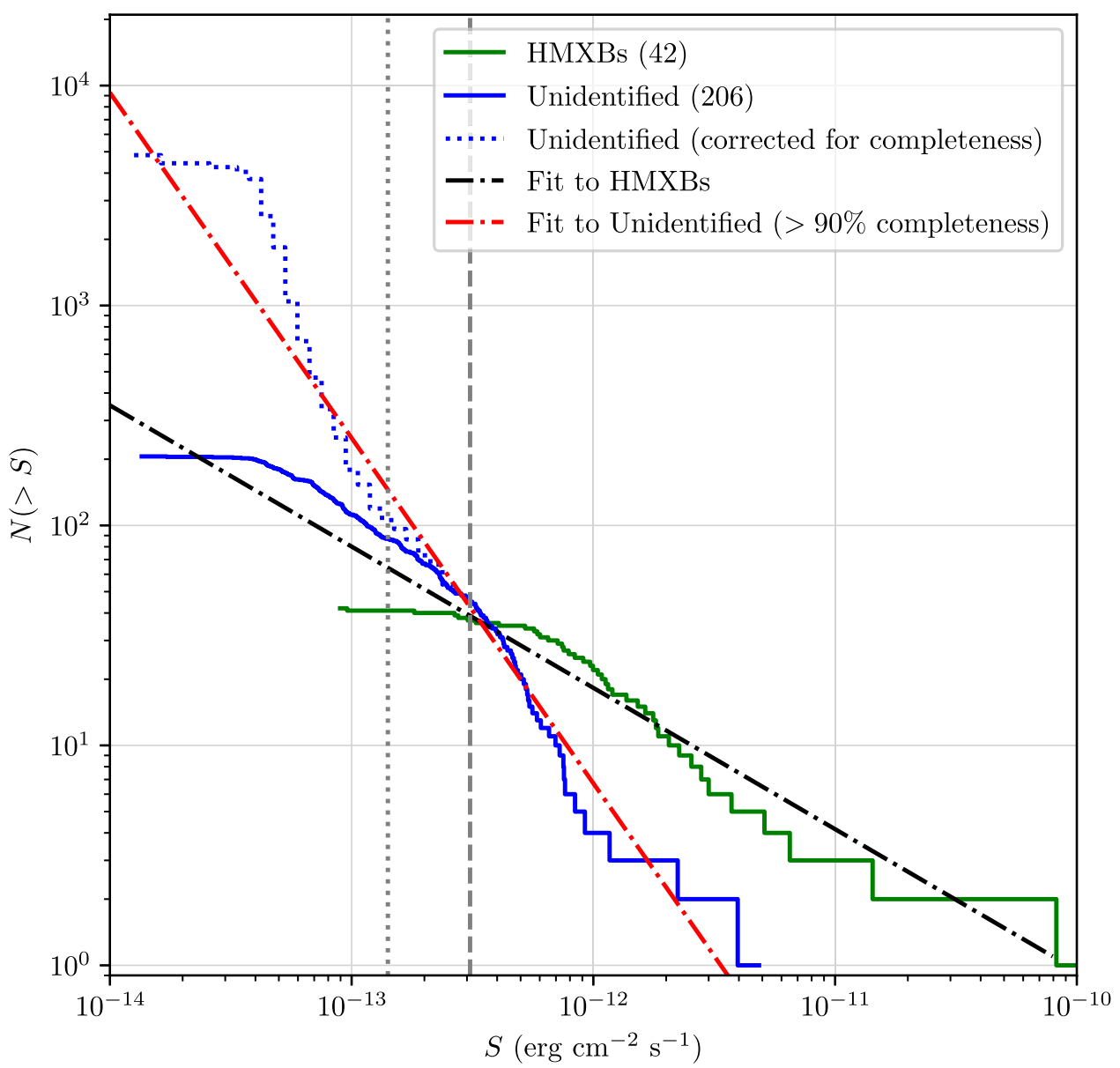

Figure 6. $\log N-\log S$ plot showing the distribution of identified HMXB sources (green) in the 1SCUBEDX catalog, compared with the log $N-\log S$ distribution of unidentified X-ray sources (blue). Dashed and dotted gray lines show the fluxes at which the 1SCUBEDX catalog is expected to be $90 \%$ and 50\% complete, respectively. Straight-line fits to the data are indicated, and for unidentified sources, we also plot the $\log N-\log S$ distribution corrected for completeness.

be due to the passage of the NS through the companion's circumstellar disk, an approximation to the DC of a source can be made using

$$
\mathrm{DC}=\frac{N_{D}}{N_{T}}
$$

where $N_{D}$ is the number of times the source was detected over $N_{T}$ total observations, based on the assumption that a typical BeXRB in the SMC is only detected by S-CUBED when it is undergoing an outburst. Due to the nature of the exposure times to each tile of the SMC, the DC cannot be taken to be completely representative of the physical nature of X-ray outbursts for SXP sources.

As the outbursting of the X-ray source is expected to take place during accretion of matter, most probably at periastron, a correlation between the DC of S-CUBED sources and the orbital period of their binary systems was searched for. The catalog of BeXRB systems in the SMC given in Table 2 of Coe \& Kirk (2015) was used to collect, where available, orbital periods of these binary systems. The plot in Figure 7 shows the DC against orbital period for these 29 systems. Though they contain a lot of scatter, there is no strong correlation between the X-ray DC and orbital period. However, given the approximate nature of utilizing the detection fraction as a proxy for DC, it is perhaps not surprising that a strong correlation is not seen, especially given the relatively sparse 7-day observation cadence of S-CUBED, combined with larger observation gaps. This incomplete coverage of SXP sources means that some outbursts may simply have been missed in observation gaps, which could well lead to underestimating the values of DC in some cases using this method.

To compare the DC with the average flux of each source, the count rates from the light curves were used. For sources with a DC $>0$, the average count rate was simply the mean of that for all observations, with nondetections contributing a 0 count $\mathrm{s}^{-1}$ value.

Figure 8 shows the relationship between the DC and the average count rate for each source, as calculated above. If a source was detected during an observation, the mean of the uncertainties in count rate was taken to contribute to the standard error in count rate for each source. The $3 \sigma$ limit for nondetections was used to determine a $1 \sigma$ uncertainty to the standard error.

Perhaps not surprisingly, there appears to be a strong correlation between the two variables: a linear regression analysis gave a Pearson correlation coefficient of $r=0.918$, and, when considering the size of the data set (32 SXP sources), a $p$-value of $\ll 0.001 \%$ was obtained, indicating a very strong positive correlation. Therefore, the frequency with which a source is detected is much more strongly driven by its average luminosity than any orbital behavior. Other physical parameters were explored, such as the temperature of the companion mass donor star or the proposed NS magnetic field 


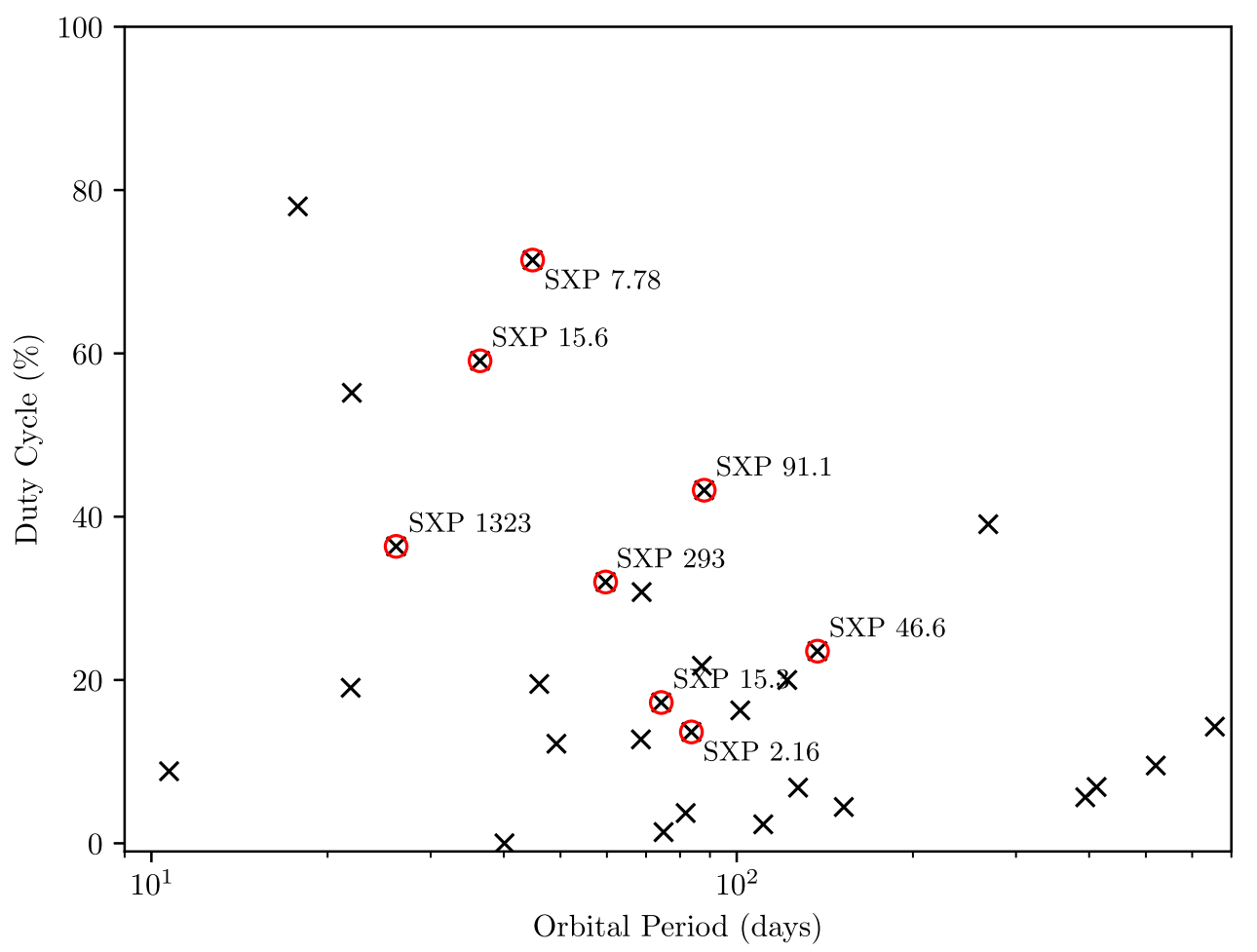

Figure 7. Percentage duty cycle of 30 SXP sources against their listed orbital periods. Sources with S-CUBED detected periods from Table 4 are highlighted with red circles and labeled with their SXP names. Note that we also highlight SXP 7.78 (=SMC X-3), as the orbital period is detected in this source after the large Type II outburst as discussed in Section 6.2.2.

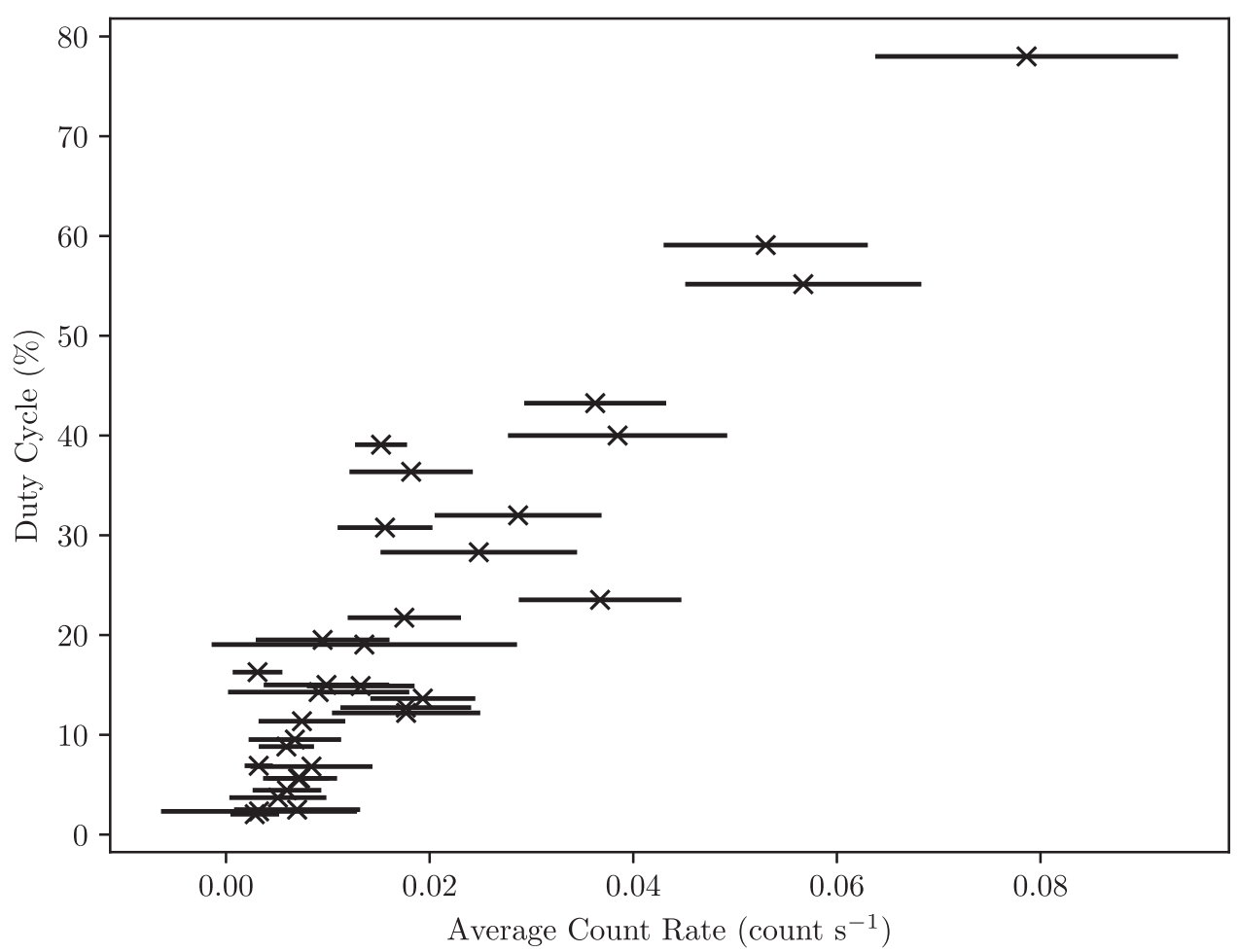

Figure 8. DC of 33 SXP sources, excluding SMC X-3 and SXP 59.0, which underwent major Type II outbursts, plotted against their mean count rate across the observation period.

(Klus et al. 2014), but none showed a strong a correlation with the DC.

We have shown that we are complete at the $90 \%$ level down to $S=3 \times 10^{-13} \mathrm{erg} \mathrm{cm}^{-2} \mathrm{~s}^{-1}$. This corresponds to $\sim 0.005$ counts s ${ }^{-1}$ in XRT. If we look at Figure 8, we can see that this $90 \%$ threshold of 0.005 counts s$^{-1}$ is on the extreme left of the plot. So all the illustrated DC points are from the brighter sources and hence must all be, at least, 90\% complete/accurate. Thus, we conclude that Figures 7 and 8 are not significantly affected by completeness concerns. 

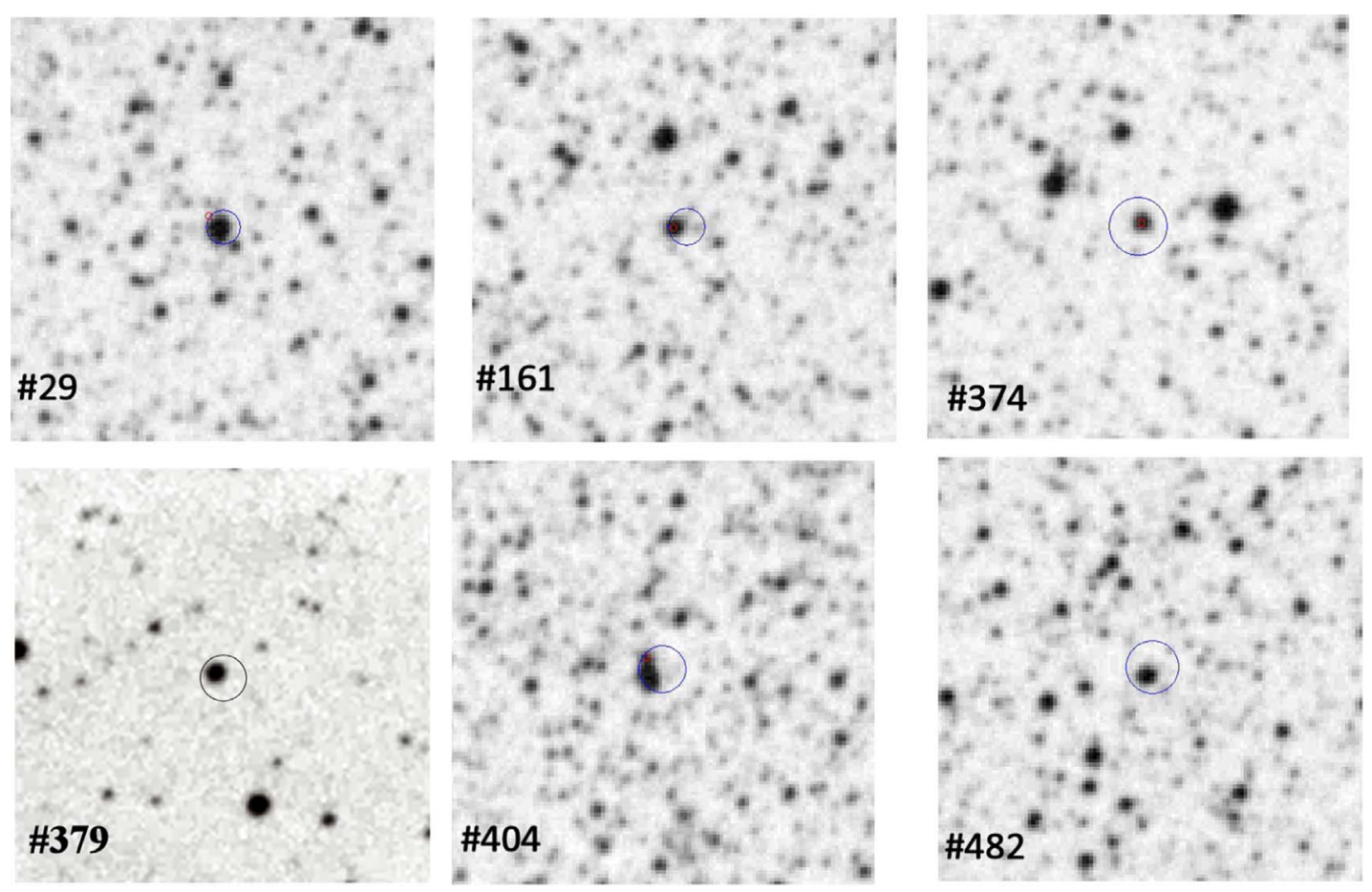

Figure 9. Finding charts for the six proposed HMXB identifications discussed in the text. Each negative image is taken from the DSS2 red survey and is $2^{\prime} \times 2^{\prime}$ in size. North is at the top, east to the right. The uncertainty in the position of the S-CUBED source is shown as a blue circle. The red dot indicates the position of the proposed optical counterpart.

\subsection{Confirmation of Proposed HMXB Systems in ISCUBEDX}

In order to identify new or confirm proposed HMXB sources, the list of definite S-CUBED detections was first cross-correlated with the list of proposed and identified HMXBs in Haberl \& Sturm (2016). Their list was produced from their X-ray mapping of the SMC with XMM-Newton and contains 148 objects they suggest are or could be identified as HMXBs. To identify a possible match between their catalog and ours, it was required that the $1 \sigma$ positional uncertainties from the two samples overlapped. The result was 29 matches between the two samples, of which 24 were confirmed SXP sources already listed in Coe \& Kirk (2015). The fields of the remaining six objects are shown in Figure 9, and the details of each of these sources are listed in Table 3. From Figure 9 it is apparent that there is an optical counterpart present within the S-CUBED positional uncertainty circle in each case. Of the six objects listed, the only one that Haberl \& Sturm (2016) doubted being identified as an HMXB is SC482. Their doubts arise from the reported lack of $\mathrm{H} \alpha$ emission in the proposed optical counterpart. This object is also in the Evans et al. (2004) catalog listed as a $\mathrm{B} 0.5 \mathrm{~V}$ star in the $\mathrm{SMC}$, similar to many HMXB systems. In addition, the OGLE III and IV light curves (Udalski et al. 1997, 2015) reveal substantial random outbursts from this system with $I$-band variations as large as $1 \mathrm{mag}$. Such behavior is symptomatic of the growth and decline of circumstellar disk structures around the star. Thus, it is very probable that $\mathrm{H} \alpha$ measurements made at the right time would reveal a substantial Balmer line excess and confirm the true nature of this object to be a Be star and hence this system to be a clear HMXB.

More generally, the error circles of all the confirmed S-CUBED objects were searched for matches within the [MA93] (Meyssonnier \& Azzopardi 1993) and AzV (Azzopardi \& Vigneau 1979) catalogs. This revealed a total of 29 matches, all of which are known SXP objects. Though this broad search did not reveal any further HMXB candidates, the possibility still remains that some of the S-CUBED objects could still correlate with $\mathrm{Be}$ stars that are not in either, or any, catalog.

In addition to positional matching, we examined the X-ray spectral signatures of these HMXB systems. We found that the mean fitted spectral index for known BeXRBs in 1SCUBEDX was $\Gamma=0.9 \pm 0.4$. For the six sources listed in Table 3, the mean spectral index was $\Gamma=0.9 \pm 0.5$, i.e., consistent with the population of known BeXRBs. In comparison, the average spectral index for all other sources in 1SCUBEDX was $\Gamma=$ $1.4 \pm 0.7$, i.e., softer, although the spread is large enough that we cannot definitely say that these represent a separate population of sources, and we cannot rule out the presence of a possible population of unidentified HMXBs.

\subsection{Orbital Period Searches}

For each S-CUBED source flagged as "Good," we performed a Lomb-Scargle (L-S) period search (e.g., Lomb 1976; Scargle 1982) of the light-curve data in the range of 21-180 days, in order to search for evidence of periodicities, perhaps associated with orbital or superorbital periods. To perform this L-S search, we utilized the implementation present in AstroPy 3.0 (Astropy Collaboration et al. 2013) and performed the search on count rate data extracted from the S-CUBED pipeline. In the case of upper limits, we assumed a count rate of zero, in order to include nondetections in the period search. For each period we calculated the false-alarm probability $\left(P_{\text {false }}\right)$ for the most significant peak in the periodogram. For the blind period search (i.e., where we do not assume that we know what the period is), we only report period detections for $P_{\text {false }} \leqslant 1 \%$, in order to avoid spurious detections.

In the case of a period detection, we estimate the error on the period measurement using a Monte Carlo technique (e.g., 
Table 2

Catalog of X-Ray Sources Detected in the First Year of S-CUBED Observations

\begin{tabular}{|c|c|c|c|c|c|c|c|c|c|}
\hline $\mathrm{SC} \#$ & $\begin{array}{c}\text { Name } \\
(1 \text { SCUBEDX J...) }\end{array}$ & $\begin{array}{l}\text { R.A./Decl. } \\
\text { (J2000) }\end{array}$ & $\begin{array}{l}\text { Err } \\
\left({ }^{\prime \prime}\right)\end{array}$ & $\begin{array}{l}\text { Det. } \\
(\%)\end{array}$ & $\begin{array}{r}f_{\text {mean }}(0.5-10 \mathrm{keV}) \\
\quad\left(\mathrm{erg} \mathrm{s}^{-1} \mathrm{~cm}^{-2}\right)\end{array}$ & $\Gamma$ & Catalog Name & $\mathrm{Cl}$. & $\begin{array}{c}\text { Type } \\
\text { (Reference) }\end{array}$ \\
\hline$\overline{\mathrm{SC} 1}$ & J011705.2-732635 & $01^{\mathrm{h}} 17^{\mathrm{m}} 05^{\mathrm{s}} .18 /-73^{\circ} 26^{\prime} 35^{\prime \prime} 8$ & $2.2^{*}$ & 100.0 & $6.3_{-0.1}^{+0.1} \times 10^{-10}$ & $1.15_{-0.03}^{+0.05}$ & SMC X-1 & $\mathrm{K}$ & HMXB (2) \\
\hline $\mathrm{SC} 2$ & J010401.3-720155 & $01^{\mathrm{h}} 04^{\mathrm{m}} 01^{\mathrm{s}} .29 /-72^{\circ} 01^{\prime} 55^{\prime \prime} 7$ & 3.8 & 100.0 & $3.3_{-0.1}^{+0.1} \times 10^{-11}$ & $6.94_{-0.29}^{+0.32}$ & 1E $0102.2-7219$ & K & SNR \\
\hline $\mathrm{SC} 3$ & J004854.9-734945 & $00^{\mathrm{h}} 48^{\mathrm{m}} 54^{\mathrm{s}} .93 /-73^{\circ} 49^{\prime} 45^{\prime \prime} 6$ & 3.8 & 59.1 & $3.2_{-0.2}^{+0.2} \times 10^{-12}$ & $0.94_{-0.15}^{+0.20}$ & SXP 15.6 & K & $\operatorname{BeXRB}(1,2$ \\
\hline $\mathrm{SC} 4$ & J003817.7-730926 & $00^{\mathrm{h}} 38^{\mathrm{m}} 17^{\mathrm{s}} .67 /-73^{\circ} 09^{\prime} 26^{\prime \prime} 6$ & 7.1 & 19.5 & $9.3_{-1.1}^{+1.2} \times 10^{-13}$ & $1.57_{-0.27}^{+0.68}$ & $\begin{array}{c}1 \mathrm{RXS} \\
\text { J003823.0-730931 }\end{array}$ & K & $\ldots$ \\
\hline SC5 & J004910.3-724939 & $00^{\mathrm{h}} 49^{\mathrm{m}} 10^{\mathrm{s}} .29 /-72^{\circ} 49^{\prime} 39^{\prime \prime} 2$ & 4.1 & 78.0 & $7.1_{-0.4}^{+0.4} \times 10^{-12}$ & $0.70_{-0.30}^{+0.30}$ & SXP 18.3 & K & $\operatorname{BeXRB}(1)$ \\
\hline SC6 & J005056.4-721333 & $00^{\mathrm{h}} 50^{\mathrm{m}} 56^{\mathrm{s}} .39 /-72^{\circ} 13^{\prime} 33^{\prime \prime} 4$ & 4.6 & 43.2 & $2.8_{-0.2}^{+0.4} \times 10^{-12}$ & $0.85_{-0.18}^{+0.23}$ & SXP 91.1 & K & $\operatorname{BeXRB}(1,2$ \\
\hline $\mathrm{SC} 7$ & J003850.6-731051 & $00^{\mathrm{h}} 38^{\mathrm{m}} 50^{\mathrm{s}} .65 /-73^{\circ} 10^{\prime} 51^{\prime \prime} 1$ & 6.5 & 16.7 & $4.3_{-0.7}^{+0.7} \times 10^{-13}$ & $2.41_{-0.74}^{+1.19}$ & $\begin{array}{l}\text { OGLE } 003850.79- \\
731053.1\end{array}$ & K & $\operatorname{AGN}(9,10)$ \\
\hline $\mathrm{SC} 8$ & J003235.5-730650 & $00^{\mathrm{h}} 32^{\mathrm{m}} 35^{\mathrm{s}} .46 /-73^{\circ} 06^{\prime} 50^{\prime \prime} 9$ & $2.3^{*}$ & 71.4 & $1.4_{-0.1}^{+0.1} \times 10^{-12}$ & $1.53_{-0.10}^{+0.20}$ & $\ldots$ & $\mathrm{U}$ & AGN \\
\hline $\mathrm{SC} 10$ & $\mathrm{~J} 005811.2-723051$ & $00^{\mathrm{h}} 58^{\mathrm{m}} 11^{\mathrm{s}} .15 /-72^{\circ} 30^{\prime} 51^{\prime \prime} 0$ & 4.6 & 32.0 & $2.2_{-0.2}^{+0.2} \times 10^{-12}$ & $0.92_{-0.24}^{+0.55}$ & SXP 293 & K & $\operatorname{BeXRB}(1)$ \\
\hline SC11 & J010336.0-720130 & $01^{\mathrm{h}} 03^{\mathrm{m}} 36^{\mathrm{s}} .00 /-72^{\circ} 01^{\prime} 30^{\prime \prime} 8$ & 5.4 & 36.4 & $1.6_{-0.2}^{+0.2} \times 10^{-12}$ & $0.72_{-0.25}^{+0.41}$ & SXP 1323 & K & BeXRB (1) \\
\hline $\mathrm{SC} 12$ & J005248.2-724914 & $00^{\mathrm{h}} 52^{\mathrm{m}} 48^{\mathrm{s}} \cdot 17 /-72^{\circ} 49^{\prime} 14^{\prime \prime} 4$ & 5.9 & 1.3 & $9.4_{-1.8}^{+2.0} \times 10^{-14}$ & $1.27_{-0.84}^{+1.90}$ & $\begin{array}{c}\text { 1SXPS } \\
\text { J005245.2-724916 }\end{array}$ & K & $\ldots$ \\
\hline $\mathrm{SC} 13$ & J005455.4-724513 & $00^{\mathrm{h}} 54^{\mathrm{m}} 55^{\mathrm{s}} .42 /-72^{\circ} 45^{\prime} 13^{\prime \prime} 1$ & 4.0 & 39.1 & $1.2_{-0.1}^{+0.1} \times 10^{-12}$ & $0.81_{-0.19}^{+0.22}$ & SXP 504 & K & $\operatorname{BeXRB}(1,2$ \\
\hline $\mathrm{SC} 14$ & J005518.1-723852 & $00^{\mathrm{h}} 55^{\mathrm{m}} 18^{\mathrm{s}} .09 /-72^{\circ} 38^{\prime} 52^{\prime \prime} 3$ & 5.3 & 6.9 & $6.1_{-0.9}^{+1.0} \times 10^{-13}$ & $-0.03_{-0.58}^{+1.42}$ & SXP 701 & K & $\operatorname{BeXRB}(1,2$ \\
\hline SC16 & J012140.6-725731 & $01^{\mathrm{h}} 21^{\mathrm{m}} 40^{\mathrm{s}} \cdot 63 /-72^{\circ} 57^{\prime} 31^{\prime \prime} \cdot 9$ & $3.2^{*}$ & 13.6 & $1.5_{-0.2}^{+0.2} \times 10^{-12}$ & $0.80_{-0.30}^{+0.40}$ & SXP 2.16 & K & $\operatorname{BeXRB}(1,2$ \\
\hline $\mathrm{SC} 17$ & J010428.3-723135 & $01^{\mathrm{h}} 04^{\mathrm{m}} 28^{\mathrm{s}} \cdot 32 /-72^{\circ} 31^{\prime} 355^{\prime \prime} 3$ & 5.1 & 28.3 & $1.9_{-0.2}^{+0.2} \times 10^{-12}$ & $1.10_{-0.60}^{+0.770}$ & $\begin{array}{c}\text { XMMU } \\
\text { J010429.4-723136 }\end{array}$ & K & $\operatorname{BeXRB}(2)$ \\
\hline $\mathrm{SC} 18$ & J005749.8-720757 & $00^{\mathrm{h}} 57^{\mathrm{m}} 49^{\mathrm{s}} \cdot 84 /-72^{\circ} 07^{\prime} 57^{\prime \prime} 4$ & 5.5 & 11.4 & $7.5_{-1.5}^{+1.7} \times 10^{-13}$ & $0.60_{-0.60}^{+0.90}$ & SXP 152 & K & $\operatorname{BeXRB}(1,2$ \\
\hline SC19 & J010042.9-721134 & $01^{\mathrm{h}} 00^{\mathrm{m}} 42^{\mathrm{s}} \cdot 92 /-72^{\circ} 11^{\prime} 34^{\prime \prime} 8$ & 6.6 & 15.4 & $3.9_{-0.4}^{+0.4} \times 10^{-13}$ & $4.00_{-1.30}^{+1.70}$ & $\begin{array}{c}\text { CXOU } \\
\text { J010043.1-721134 }\end{array}$ & K & $\begin{array}{c}\text { Magentar } \\
\text { (13) }\end{array}$ \\
\hline $\mathrm{SC} 20$ & J005919.8-722317 & $00^{\mathrm{h}} 59^{\mathrm{m}} 19^{\mathrm{s}} .82 /-72^{\circ} 23^{\prime} 17^{\prime \prime} \cdot 9$ & 4.3 & 40.0 & $2.3_{-0.2}^{+0.2} \times 10^{-12}$ & $1.20_{-0.30}^{+0.50}$ & SXP 202A & K & BeXRB (1) \\
\hline $\mathrm{SC} 25$ & $\mathrm{~J} 012140.5-725952$ & $01^{\mathrm{h}} 21^{\mathrm{m}} 40^{\mathrm{s}} \cdot 49 /-72^{\circ} 59^{\prime} 52^{\prime \prime} 0$ & 5.8 & 0.0 & $1.9_{-1.1}^{+1.4} \times 10^{-13}$ & $\ldots$ & $\ldots$ & $\mathrm{U}$ & $\ldots$ \\
\hline $\mathrm{SC} 27$ & J005819.7-725107 & $00^{\mathrm{h}} 58^{\mathrm{m}} 19^{\mathrm{s}} \cdot 70 /-72^{\circ} 51^{\prime} 07^{\prime !} 4$ & 7.1 & 2.1 & $4.7_{-1.9}^{+2.3} \times 10^{-14}$ & $\ldots$ & $\ldots$ & $\mathrm{U}$ & $\ldots$ \\
\hline $\mathrm{SC} 28$ & J005231.3-725017 & $00^{\mathrm{h}} 52^{\mathrm{m}} 31^{\mathrm{s}} .33 /-72^{\circ} 50^{\prime} 17^{\prime \prime} 6$ & 4.6 & 0.0 & $5.2_{-3.2}^{+4.0} \times 10^{-14}$ & $\ldots$ & $\ldots$ & $\mathrm{U}$ & $\ldots$ \\
\hline $\operatorname{SC} 29$ & J010155.5-723236 & $01^{\mathrm{h}} 01^{\mathrm{m}} 55^{\mathrm{s}} \cdot 53 /-72^{\circ} 32^{\prime} 36^{\prime \prime} 0$ & 4.9 & 7.3 & $6.9_{-1.3}^{+1.5} \times 10^{-13}$ & $1.00_{-1.10}^{+1.60}$ & AvZ 285 & K & $\operatorname{HMXB}(2,3)$ \\
\hline $\mathrm{SC} 30$ & J005427.7-725722 & $00^{\mathrm{h}} 54^{\mathrm{m}} 27^{\mathrm{s}} \cdot 68 /-72^{\circ} 57^{\prime} 22^{\prime \prime} 9$ & 5.0 & 0.0 & $9.9_{-5.2}^{+6.5} \times 10^{-14}$ & $\ldots$ & $\cdots$ & $\mathrm{U}$ & $\cdots$ \\
\hline $\mathrm{SC} 32$ & J011838.2-732533 & $01^{\mathrm{h}} 18^{\mathrm{m}} 38^{\mathrm{s}} .21 /-73^{\circ} 25^{\prime} 33^{\prime \prime} 7$ & $7.1^{*}$ & 47.5 & $5.5_{-0.5}^{+0.5} \times 10^{-13}$ & $4.37_{-0.55}^{+1.19}$ & HD $8191 \mathrm{~A} / \mathrm{B}$ & K & FG-star (12) \\
\hline $\mathrm{SC} 33$ & J012746.3-733303 & $01^{\mathrm{h}} 27^{\mathrm{m}} 46^{\mathrm{s}} .32 /-73^{\circ} 33^{\prime} 03^{\prime \prime} 0$ & 6.3 & 14.3 & $1.8_{-0.3}^{+0.3} \times 10^{-12}$ & $0.40_{-1.50}^{+1.90}$ & SXP 1062 & K & $\operatorname{BeXRB}(1)$ \\
\hline $\mathrm{SC} 34$ & J005254.7-715802 & $00^{\mathrm{h}} 52^{\mathrm{m}} 54^{\mathrm{s}} \cdot 69 /-71^{\circ} 58^{\prime} 02^{\prime \prime} 5$ & 4.9 & 12.7 & $1.2_{-0.1}^{+0.1} \times 10^{-12}$ & $1.00_{-0.40}^{+0.70}$ & SXP 169 & K & $\operatorname{BeXRB}(1,2$ \\
\hline $\mathrm{SC} 36$ & J010817.8-730357 & $01^{\mathrm{h}} 08^{\mathrm{m}} 17^{\mathrm{s}} \cdot 79 /-73^{\circ} 03^{\prime} 57^{\prime \prime} 6$ & 6.3 & 1.4 & $6.8_{-2.2}^{+2.6} \times 10^{-14}$ & $\ldots$ & $\ldots$ & $\mathrm{U}$ & $\ldots$ \\
\hline $\mathrm{SC} 38$ & J003312.5-731610 & $00^{\mathrm{h}} 33^{\mathrm{m}} 12^{\mathrm{s}} \cdot 54 /-73^{\circ} 16^{\prime} 10^{\prime \prime} 4$ & 5.0 & 1.1 & $2.1_{-0.7}^{+0.9} \times 10^{-14}$ & $\ldots$ & $\ldots$ & $\mathrm{U}$ & $\ldots$ \\
\hline SC39 & J003619.9-731903 & $00^{\mathrm{h}} 36^{\mathrm{m}} 19^{\mathrm{s}} .88 /-73^{\circ} 19^{\prime} 03^{\prime \prime} 3$ & 5.1 & 2.9 & $5.8_{-2.2}^{+2.7} \times 10^{-14}$ & $\ldots$ & $\ldots$ & $\mathrm{U}$ & $\ldots$ \\
\hline $\mathrm{SC} 41$ & J004427.0-731319 & $00^{\mathrm{h}} 44^{\mathrm{m}} 27^{\mathrm{s}} .04 /-73^{\circ} 13^{\prime} 19^{\prime \prime} 4$ & 7.1 & 2.5 & $4.0_{-1.6}^{+2.0} \times 10^{-14}$ & $\ldots$ & $\ldots$ & $\mathrm{U}$ & $\ldots$ \\
\hline $\mathrm{SC} 43$ & J004722.0-731224 & $00^{\mathrm{h}} 47^{\mathrm{m}} 22^{\mathrm{s}} \cdot 04 /-73^{\circ} 12^{\prime} 24^{\prime \prime} 8$ & 7.7 & 12.2 & $1.7_{-0.2}^{+0.2} \times 10^{-12}$ & $0.70_{-0.50}^{+0.80}$ & SXP 264 & K & $\operatorname{BeXRB}(1,2$ \\
\hline $\mathrm{SC} 45$ & J004708.1-725303 & $00^{\mathrm{h}} 47^{\mathrm{m}} 08^{\mathrm{s}} .08 /-72^{\circ} 53^{\prime} 03^{\prime \prime} 5$ & 5.4 & 0.0 & $3.1_{-1.4}^{+1.8} \times 10^{-13}$ & $\ldots$ & $\ldots$ & $\mathrm{U}$ & $\ldots$ \\
\hline SC46 & J003046.8-725850 & $00^{\mathrm{h}} 30^{\mathrm{m}} 46^{\mathrm{s}} .84 /-72^{\circ} 58^{\prime} 50^{\prime \prime} 4$ & 4.9 & 0.0 & $6.3_{-3.9}^{+5.0} \times 10^{-14}$ & $\ldots$ & $\ldots$ & $\mathrm{U}$ & $\ldots$ \\
\hline $\mathrm{SC} 48$ & J012634.3-725147 & $01^{\mathrm{h}} 26^{\mathrm{m}} 34^{\mathrm{s}} .28 /-72^{\circ} 51^{\prime} 47^{\prime \prime} 5$ & 4.6 & 1.5 & $5.5_{-2.0}^{+2.5} \times 10^{-14}$ & $\ldots$ & $\cdots$ & $\mathrm{U}$ & $\ldots$ \\
\hline SC49 & J010252.2-724433 & $01^{\mathrm{h}} 02^{\mathrm{m}} 52^{\mathrm{s}} \cdot 17 /-72^{\circ} 44^{\prime} 33^{\prime \prime} 4$ & 4.4 & 55.2 & $4.3_{-0.3}^{+0.3} \times 10^{-12}$ & $0.88_{-0.24}^{+0.38}$ & SXP 6.85 & K & BeXRB (1) \\
\hline SC51 & J005852.8-724927 & $00^{\mathrm{h}} 58^{\mathrm{m}} 52^{\mathrm{s}} .83 /-72^{\circ} 49^{\prime} 27^{\prime \prime} 0$ & 5.0 & 3.2 & $5.1_{-2.0}^{+2.5} \times 10^{-14}$ & $\ldots$ & $\ldots$ & $\mathrm{U}$ & $\ldots$ \\
\hline $\operatorname{SC} 52$ & J012252.0-724151 & $01^{\mathrm{h}} 22^{\mathrm{m}} 51^{\mathrm{s}} .97 /-72^{\circ} 41^{\prime} 51^{\prime \prime} 3$ & 6.3 & 5.4 & $1.3_{-0.4}^{+0.4} \times 10^{-13}$ & $\ldots$ & $\ldots$ & U & $\ldots$ \\
\hline SC54 & J012126.1-725823 & $01^{\mathrm{h}} 21^{\mathrm{m}} 26^{\mathrm{s}} .07 /-72^{\circ} 58^{\prime} 23^{\prime \prime} 2$ & 5.8 & 3.1 & $1.3_{-0.2}^{+0.2} \times 10^{-13}$ & $4.20_{-1.40}^{+3.70}$ & $\begin{array}{c}\text { 3XMM } \\
\text { J012124.7-725827 }\end{array}$ & K & $\ldots$ \\
\hline SC55 & J004151.3-724255 & $00^{\mathrm{h}} 41^{\mathrm{m}} 51^{\mathrm{s}} .33 /-72^{\circ} 42^{\prime} 55^{\prime \prime} 5$ & 5.0 & 4.7 & $1.5_{-0.4}^{+0.4} \times 10^{-13}$ & $\ldots$ & $\cdots$ & $\mathrm{U}$ & $\ldots$ \\
\hline SC56 & J004025.5-720810 & $00^{\mathrm{h}} 40^{\mathrm{m}} 25^{\mathrm{s}} .49 /-72^{\circ} 08^{\prime} 10^{\prime \prime} 4$ & 6.6 & 0.0 & $5.3_{-2.4}^{+3.2} \times 10^{-13}$ & $\ldots$ & $\ldots$ & $\mathrm{U}$ & $\ldots$ \\
\hline $\operatorname{SC} 58$ & J004454.9-723156 & $00^{\mathrm{h}} 44^{\mathrm{m}} 54^{\mathrm{s}} .92 /-72^{\circ} 31^{\prime} 56^{\prime \prime} 6$ & 5.9 & 1.8 & $6.9_{-2.2}^{+2.5} \times 10^{-14}$ & $\ldots$ & $\ldots$ & $\mathrm{U}$ & $\ldots$ \\
\hline SC59 & J004820.4-722739 & $00^{\mathrm{h}} 48^{\mathrm{m}} 20^{\mathrm{s}} \cdot 38 /-72^{\circ} 27^{\prime} 39^{\prime \prime} 1$ & 4.9 & 3.9 & $9.5_{-3.0}^{+3.5} \times 10^{-14}$ & $\ldots$ & $\ldots$ & $\mathrm{U}$ & $\ldots$ \\
\hline SC61 & J004720.5-724557 & $00^{\mathrm{h}} 47^{\mathrm{m}} 20^{\mathrm{s}} \cdot 48 /-72^{\circ} 45^{\prime} 57^{\prime \prime} 4$ & 5.3 & 1.8 & $2.4_{-1.2}^{+1.5} \times 10^{-13}$ & $\ldots$ & $\ldots$ & $\mathrm{U}$ & $\ldots$ \\
\hline SC64 & J005116.2-721655 & $00^{\mathrm{h}} 51^{\mathrm{m}} 16^{\mathrm{s}} .20 /-72^{\circ} 16^{\prime} 55^{\prime \prime} 8$ & 6.8 & 10.9 & $4.6_{-0.7}^{+0.7} \times 10^{-13}$ & $2.00_{-0.30}^{+0.60}$ & $\begin{array}{c}\text { MACHO } \\
208.16034 .100\end{array}$ & K & AGN (9) \\
\hline SC66 & J004637.9-724919 & $00^{\mathrm{h}} 46^{\mathrm{m}} 37^{\mathrm{s}} \cdot 91 /-72^{\circ} 49^{\prime} 19^{\prime \prime} 4$ & 5.6 & 0.0 & $4.5_{-4.5}^{+5.8} \times 10^{-14}$ & $\cdots$ & $\cdots$ & $\mathrm{U}$ & $\ldots$ \\
\hline SC67 & J005244.6-723617 & $00^{\mathrm{h}} 52^{\mathrm{m}} 44^{\mathrm{s}} \cdot 60 /-72^{\circ} 36^{\prime} 17^{\prime \prime} \cdot 7$ & 4.4 & 3.0 & $4.4_{-1.0}^{+1.1} \times 10^{-14}$ & $\ldots$ & 1WGA J0052.8-7236 & K & \\
\hline SC68 & J005053.3-724924 & $00^{\mathrm{h}} 50^{\mathrm{m}} 53^{\mathrm{s}} .32 /-72^{\circ} 49^{\prime} 24^{\prime \prime} 6$ & 5.3 & 2.5 & $4.5_{-1.0}^{+1.2} \times 10^{-14}$ & $\ldots$ & $\ldots$ & $\mathrm{U}$ & $\ldots$ \\
\hline SC71 & J005205.3-722603 & $00^{\mathrm{h}} 52^{\mathrm{m}} 05^{\mathrm{s}} .27 /-72^{\circ} 26^{\prime} 03^{\prime \prime} 8$ & 4.3 & 71.4 & $1.4_{-0.0}^{+0.0} \times 10^{-10}$ & $1.16_{-0.05}^{+0.05}$ & SMC X-3 & K & $\operatorname{BeXRB}(1,2$ \\
\hline SC72 & J005606.0-722749 & $00^{\mathrm{h}} 56^{\mathrm{m}} 06^{\mathrm{s}} .04 /-72^{\circ} 27^{\prime} 49^{\prime \prime} 9$ & 5.2 & 1.7 & $8.1_{-2.9}^{+3.4} \times 10^{-14}$ & $\ldots$ & $\ldots$ & $\mathrm{U}$ & $\ldots$ \\
\hline SC73 & J011232.6-720613 & $01^{\mathrm{h}} 12^{\mathrm{m}} 32^{\mathrm{s}} .58 /-72^{\circ} 06^{\prime} 13^{\prime \prime} 2$ & 5.5 & 1.6 & $7.3_{-2.4}^{+2.9} \times 10^{-14}$ & $\ldots$ & $\ldots$ & U & $\ldots$ \\
\hline SC75 & J010027.4-723907 & $01^{\mathrm{h}} 00^{\mathrm{m}} 27^{\mathrm{s}} .43 /-72^{\circ} 39^{\prime} 07^{\prime \prime} 4$ & 5.1 & 4.2 & $5.0_{-1.7}^{+2.1} \times 10^{-14}$ & $\ldots$ & 1WGA J0100.6-7239 & K & $\ldots$ \\
\hline SC77 & J005321.3-730118 & $00^{\mathrm{h}} 53^{\mathrm{m}} 21^{\mathrm{s}} .27 /-73^{\circ} 01^{\prime} 18^{\prime \prime} 8$ & 6.8 & 2.3 & $5.4_{-2.0}^{+2.5} \times 10^{-13}$ & $\ldots$ & & $\mathrm{U}$ & $\ldots$ \\
\hline
\end{tabular}


Table 2

(Continued)

\begin{tabular}{|c|c|c|c|c|c|c|c|c|c|}
\hline $\mathrm{SC} \#$ & $\begin{array}{c}\text { Name } \\
(1 \text { SCUBEDX J...) }\end{array}$ & $\begin{array}{l}\text { R.A./Decl. } \\
\text { (J2000) }\end{array}$ & $\begin{array}{l}\text { Err } \\
\left({ }^{\prime \prime}\right)\end{array}$ & $\begin{array}{l}\text { Det. } \\
(\%)\end{array}$ & $\begin{array}{r}f_{\text {mean }}(0.5-10 \mathrm{keV}) \\
\left(\mathrm{erg} \mathrm{s}^{-1} \mathrm{~cm}^{-2}\right)\end{array}$ & $\Gamma$ & Catalog Name & $\mathrm{Cl}$. & $\begin{array}{c}\text { Type } \\
\text { (Reference) }\end{array}$ \\
\hline SC79 & J005534.1-722910 & $00^{\mathrm{h}} 55^{\mathrm{m}} 34^{\mathrm{s}} .06 /-72^{\circ} 29^{\prime} 10^{\prime \prime} .9$ & 5.4 & 5.6 & $7.5_{-1.3}^{+1.4} \times 10^{-13}$ & $0.53_{-0.63}^{+1.06}$ & SXP 645 & $\mathrm{~K}$ & $\overline{B e X R B ~(1) ~}$ \\
\hline SC 80 & J005052.5-710902 & $00^{\mathrm{h}} 50^{\mathrm{m}} 52^{\mathrm{s}} \cdot 47 /-71^{\circ} 09^{\prime} 02^{\prime \prime} 3$ & 6.8 & 59.5 & $7.5_{-0.6}^{+0.7} \times 10^{-13}$ & $8.40_{-1.90}^{+2.40}$ & HD 5028 & $\mathrm{~K}$ & FG star \\
\hline SC82 & J010450.6-722322 & $01^{\mathrm{h}} 04^{\mathrm{m}} 50^{\mathrm{s}} \cdot 56 /-72^{\circ} 23^{\prime} 22^{\prime \prime} .4$ & 6.0 & 4.9 & $2.1_{-0.5}^{+0.5} \times 10^{-13}$ & $\ldots$ & SNR B0103-72.6 & $\mathrm{K}$ & SNR \\
\hline SC84 & J003447.2-725110 & $00^{\mathrm{h}} 34^{\mathrm{m}} 47^{\mathrm{s}} \cdot 23 /-72^{\circ} 51^{\prime} 10^{\prime \prime} \cdot 9$ & 5.0 & 2.4 & $4.5_{-1.8}^{+2.2} \times 10^{-14}$ & $\cdots$ & $\ldots$ & $\mathrm{U}$ & $\ldots$ \\
\hline SC85 & J002829.4-731159 & $00^{\mathrm{h}} 28^{\mathrm{m}} 29^{\mathrm{s}} .40 /-73^{\circ} 11^{\prime} 59^{\prime \prime} 8$ & 6.6 & 2.6 & $6.2_{-2.4}^{+3.0} \times 10^{-13}$ & $\cdots$ & $\cdots$ & $\mathrm{U}$ & $\cdots$ \\
\hline SC86 & J012130.6-730455 & $01^{\mathrm{h}} 21^{\mathrm{m}} 30^{\mathrm{s}} \cdot 63 /-73^{\circ} 04^{\prime} 55^{\prime \prime} \cdot 9$ & 5.6 & 2.6 & $7.4_{-2.4}^{+2.9} \times 10^{-14}$ & $\cdots$ & $\cdots$ & $\mathrm{U}$ & $\cdots$ \\
\hline SC87 & J010105.3-734204 & $01^{\mathrm{h}} 01^{\mathrm{m}} 05^{\mathrm{s}} .27 /-73^{\circ} 42^{\prime} 04^{\prime \prime} 0$ & 6.7 & 3.1 & $6.4_{-1.2}^{+1.4} \times 10^{-13}$ & $0.40_{-1.10}^{+2.00}$ & $\begin{array}{c}\text { MQS J010104.72- } \\
734159.9\end{array}$ & $\mathrm{U}$ & AGN (8) \\
\hline SC89 & J011207.2-730116 & $01^{\mathrm{h}} 12^{\mathrm{m}} 07^{\mathrm{s}} \cdot 20 /-73^{\circ} 01^{\prime} 16^{\prime \prime} \cdot 2$ & 4.8 & 2.9 & $8.6_{-2.5}^{+2.9} \times 10^{-14}$ & $\ldots$ & 1WGA J0112.2-7301 & $\mathrm{K}$ & $\ldots$ \\
\hline SC91 & J011622.1-730627 & $01^{\mathrm{h}} 16^{\mathrm{m}} 22^{\mathrm{s}} \cdot 11 /-73^{\circ} 06^{\prime} 27^{\prime \prime} \cdot 7$ & 5.7 & 2.6 & $5.5_{-2.0}^{+2.5} \times 10^{-14}$ & $\ldots$ & $\ldots$ & $\mathrm{U}$ & $\ldots$ \\
\hline SC92 & J010805.3-730231 & $01^{\mathrm{h}} 08^{\mathrm{m}} 05^{\mathrm{s}} \cdot 35 /-73^{\circ} 02^{\prime} 31^{\prime \prime} .5$ & 5.3 & 0.0 & $1.7_{-1.0}^{+1.3} \times 10^{-13}$ & $\ldots$ & $\cdots$ & $\mathrm{U}$ & $\ldots$ \\
\hline SC93 & J011938.3-725609 & $01^{\mathrm{h}} 19^{\mathrm{m}} 38^{\mathrm{s}} .26 /-72^{\circ} 56^{\prime} 09^{\prime \prime} 6$ & 5.8 & 1.6 & $8.4_{-2.8}^{+3.2} \times 10^{-14}$ & $\cdots$ & $\cdots$ & $\mathrm{U}$ & $\cdots$ \\
\hline SC94 & J011445.6-733421 & $01^{\mathrm{h}} 14^{\mathrm{m}} 45^{\mathrm{s}} .57 /-73^{\circ} 34^{\prime} 21^{\prime \prime} \cdot 9$ & 7.5 & 1.4 & $5.1_{-1.8}^{+2.2} \times 10^{-14}$ & $\cdots$ & $\cdots$ & $\mathrm{U}$ & $\cdots$ \\
\hline SC95 & J010841.2-731057 & $01^{\mathrm{h}} 08^{\mathrm{m}} 41^{\mathrm{s}} .22 /-73^{\circ} 10^{\prime} 57^{\prime \prime} \cdot 5$ & 10.6 & 2.3 & $5.3_{-1.8}^{+2.2} \times 10^{-14}$ & $\ldots$ & $\ldots$ & $\mathrm{U}$ & $\ldots$ \\
\hline SC96 & J011243.3-724246 & $01^{\mathrm{h}} 12^{\mathrm{m}} 43^{\mathrm{s}} \cdot 30 /-72^{\circ} 42^{\prime} 46^{\prime \prime} \cdot 3$ & 5.5 & 2.9 & $9.3_{-2.7}^{+3.1} \times 10^{-14}$ & $\ldots$ & $\cdots$ & $\mathrm{U}$ & $\ldots$ \\
\hline SC97 & J012108.3-730713 & $01^{\mathrm{h}} 21^{\mathrm{m}} 08^{\mathrm{s}} \cdot 26 /-73^{\circ} 07^{\prime} 13^{\prime \prime} \cdot 2$ & 6.0 & 5.0 & $1.9_{-0.5}^{+0.6} \times 10^{-13}$ & $1.80_{-1.40}^{+3.20}$ & $\begin{array}{c}\text { MQS J012108.42- } \\
730713.1\end{array}$ & $\mathrm{~K}$ & AGN (8) \\
\hline SC99 & J012214.0-725858 & $01^{\mathrm{h}} 22^{\mathrm{m}} 14^{\mathrm{s}} \cdot 02 /-72^{\circ} 58^{\prime} 58^{\prime \prime} \cdot 2$ & 7.2 & 2.1 & $5.0_{-2.8}^{+3.5} \times 10^{-14}$ & $\ldots$ & $\ldots$ & $\mathrm{U}$ & $\ldots$ \\
\hline SC103 & J005329.3-730356 & $00^{\mathrm{h}} 53^{\mathrm{m}} 29^{\mathrm{s}} \cdot 31 /-73^{\circ} 03^{\prime} 56^{\prime \prime} \cdot 9$ & 5.4 & 2.6 & $5.7_{-1.9}^{+2.4} \times 10^{-14}$ & $\cdots$ & $\cdots$ & $\mathrm{U}$ & $\cdots$ \\
\hline $\mathrm{SC} 104$ & J005233.6-731504 & $00^{\mathrm{h}} 52^{\mathrm{m}} 33^{\mathrm{s}} .56 /-73^{\circ} 15^{\prime} 04^{\prime \prime} 0$ & 5.3 & 5.0 & $5.7_{-1.8}^{+2.1} \times 10^{-14}$ & $\ldots$ & $\ldots$ & $\mathrm{U}$ & $\cdots$ \\
\hline SC105 & J004929.3-731102 & $00^{\mathrm{h}} 49^{\mathrm{m}} 29^{\mathrm{s}} \cdot 31 /-73^{\circ} 11^{\prime} 02^{\prime \prime} .2$ & 6.9 & 15.0 & $8.3_{-1.4}^{+1.5} \times 10^{-13}$ & $1.10_{-0.84}^{+1.45}$ & SXP 893 & $\mathrm{~K}$ & $\operatorname{BeXRB}(1,2)$ \\
\hline SC107 & J004417.9-731414 & $00^{\mathrm{h}} 44^{\mathrm{m}} 17^{\mathrm{s}} .85 /-73^{\circ} 14^{\prime} 14^{\prime \prime} 6$ & 6.5 & 5.1 & $4.7_{-1.7}^{+2.1} \times 10^{-14}$ & $\cdots$ & $\cdots$ & $\mathrm{U}$ & $\cdots$ \\
\hline SC110 & J003933.5-724528 & $00^{\mathrm{h}} 39^{\mathrm{m}} 33^{\mathrm{s}} .45 /-72^{\circ} 45^{\prime} 28^{\prime \prime} 0$ & 5.4 & 2.9 & $4.1_{-1.8}^{+2.2} \times 10^{-14}$ & $\cdots$ & $\cdots$ & $\mathrm{U}$ & $\cdots$ \\
\hline SC115 & J005815.0-721806 & $00^{\mathrm{h}} 58^{\mathrm{m}} 15^{\mathrm{s}} \cdot 04 /-72^{\circ} 18^{\prime} 06^{\prime \prime} 6$ & 6.1 & 6.0 & $1.9_{-0.4}^{+0.5} \times 10^{-13}$ & $1.90_{-1.30}^{+2.10}$ & $\begin{array}{c}\text { 1SXPS J005816.6- } \\
721801\end{array}$ & K & $\ldots$ \\
\hline SC117 & J012829.5-722412 & $01^{\mathrm{h}} 28^{\mathrm{m}} 29^{\mathrm{s}} .54 /-72^{\circ} 24^{\prime} 12^{\prime \prime} 0$ & 6.1 & 0.0 & $3.1_{-1.1}^{+1.4} \times 10^{-12}$ & $\cdots$ & $\cdots$ & $\mathrm{U}$ & $\ldots$ \\
\hline $\mathrm{SC} 121$ & J010939.7-713610 & $01^{\mathrm{h}} 09^{\mathrm{m}} 39^{\mathrm{s}} \cdot 71 /-71^{\circ} 36^{\prime} 10^{\prime \prime} 6$ & 15.9 & 4.9 & $7.3_{-2.4}^{+3.0} \times 10^{-14}$ & $\cdots$ & $\cdots$ & $\mathrm{U}$ & $\cdots$ \\
\hline $\mathrm{SC} 122$ & J010238.1-721130 & $01^{\mathrm{h}} 02^{\mathrm{m}} 38^{\mathrm{s}} .06 /-72^{\circ} 11^{\prime} 30^{\prime \prime} 8$ & 10.6 & 5.3 & $7.5_{-2.2}^{+2.6} \times 10^{-14}$ & $\ldots$ & $\ldots$ & $\mathrm{U}$ & $\ldots$ \\
\hline $\mathrm{SC} 123$ & J005913.4-722234 & $00^{\mathrm{h}} 59^{\mathrm{m}} 13^{\mathrm{s}} \cdot 41 /-72^{\circ} 22^{\prime} 34^{\prime \prime} 0$ & 6.3 & 10.9 & $4.7_{-0.6}^{+0.7} \times 10^{-13}$ & $2.00_{-0.40}^{+1.00}$ & $\begin{array}{c}\text { 1SXPS J005913.7- } \\
722233\end{array}$ & K & $\ldots$ \\
\hline $\mathrm{SC} 125$ & J012353.8-723430 & $01^{\mathrm{h}} 23^{\mathrm{m}} 53^{\mathrm{s}} .79 /-72^{\circ} 34^{\prime} 30^{\prime \prime} 7$ & 7.0 & 11.9 & $4.9_{-0.6}^{+0.7} \times 10^{-13}$ & $1.45_{-0.29}^{+0.56}$ & $\cdots$ & $\mathrm{U}$ & $\cdots$ \\
\hline SC127 & J013450.7-741139 & $01^{\mathrm{h}} 34^{\mathrm{m}} 50^{\mathrm{s}} \cdot 73 /-74^{\circ} 11^{\prime} 39^{\prime \prime} \cdot 2$ & 6.2 & 14.7 & $3.0_{-0.7}^{+0.8} \times 10^{-13}$ & $\cdots$ & $\begin{array}{c}\text { 3XMM J013452.1- } \\
741136\end{array}$ & $\mathrm{~K}$ & $\cdots$ \\
\hline SC131 & J010203.7-715130 & $01^{\mathrm{h}} 02^{\mathrm{m}} 03^{\mathrm{s}} .72 /-71^{\circ} 51^{\prime} 30^{\prime \prime} 6$ & 5.3 & 4.3 & $4.1_{-1.6}^{+2.0} \times 10^{-14}$ & $\cdots$ & $\ldots$ & $\mathrm{U}$ & $\cdots$ \\
\hline $\mathrm{SC} 132$ & J010134.9-715840 & $01^{\mathrm{h}} 01^{\mathrm{m}} 34^{\mathrm{s}} .85 /-71^{\circ} 58^{\prime} 40^{\prime \prime} 1$ & 9.6 & 2.4 & $2.6_{-1.5}^{+2.0} \times 10^{-13}$ & $\ldots$ & $\begin{array}{c}\text { 3XMM J010136.5- } \\
715833\end{array}$ & K & $\ldots$ \\
\hline SC133 & J010022.9-720109 & $01^{\mathrm{h}} 00^{\mathrm{m}} 22^{\mathrm{s}} \cdot 92 /-72^{\circ} 01^{\prime} 09^{\prime \prime} .3$ & 4.8 & 2.3 & $4.1_{-1.2}^{+1.5} \times 10^{-14}$ & $\cdots$ & 1WGA J0100.3-7201 & $\mathrm{K}$ & $\cdots$ \\
\hline SC134 & J005233.2-712000 & $00^{\mathrm{h}} 52^{\mathrm{m}} 33^{\mathrm{s}} .22 /-71^{\circ} 20^{\prime} 00^{\prime \prime} 0$ & 6.4 & 1.6 & $6.9_{-2.3}^{+2.8} \times 10^{-14}$ & $\ldots$ & $\ldots$ & $\mathrm{U}$ & $\ldots$ \\
\hline SC136 & J003041.6-724929 & $00^{\mathrm{h}} 30^{\mathrm{m}} 41^{\mathrm{s}} .65 /-72^{\circ} 49^{\prime} 29^{\prime \prime} .5$ & 8.4 & 2.7 & $2.2_{-1.1}^{+1.5} \times 10^{-13}$ & $\ldots$ & $\ldots$ & $\mathrm{U}$ & $\ldots$ \\
\hline SC139 & J003252.9-730430 & $00^{\mathrm{h}} 32^{\mathrm{m}} 52^{\mathrm{s}} \cdot 93 /-73^{\circ} 04^{\prime} 30^{\prime \prime} \cdot 4$ & 5.7 & 1.1 & $3.2_{-0.7}^{+0.8} \times 10^{-14}$ & $2.60_{-0.90}^{+4.30}$ & $\ldots$ & $\mathrm{U}$ & $\ldots$ \\
\hline SC140 & J003157.6-731726 & $00^{\mathrm{h}} 31^{\mathrm{m}} 57^{\mathrm{s}} \cdot 64 /-73^{\circ} 17^{\prime} 26^{\prime \prime} \cdot 5$ & 5.8 & 5.7 & $5.2_{-0.9}^{+1.0} \times 10^{-14}$ & $3.30_{-1.50}^{+3.00}$ & $\cdots$ & $\mathrm{U}$ & $\cdots$ \\
\hline SC141 & J003344.3-730743 & $00^{\mathrm{h}} 33^{\mathrm{m}} 44^{\mathrm{s}} .33 /-73^{\circ} 07^{\prime} 43^{\prime \prime} 8$ & 4.5 & 7.4 & $6.9_{-1.2}^{+1.3} \times 10^{-14}$ & $\ldots$ & $\ldots$ & $\mathrm{U}$ & $\ldots$ \\
\hline SC143 & J003108.2-731207 & $00^{\mathrm{h}} 31^{\mathrm{m}} 08^{\mathrm{s}} \cdot 18 /-73^{\circ} 12^{\prime} 07^{\prime \prime} \cdot 3$ & 4.1 & 20.8 & $1.2_{-0.1}^{+0.1} \times 10^{-13}$ & $4.80_{-1.20}^{+1.80}$ & $\ldots$ & $\mathrm{U}$ & $\ldots$ \\
\hline SC145 & J002940.4-731013 & $00^{\mathrm{h}} 29^{\mathrm{m}} 40^{\mathrm{s}} \cdot 35 /-73^{\circ} 10^{\prime} 13^{\prime \prime} \cdot 3$ & 6.9 & 0.0 & $2.2_{-1.0}^{+1.3} \times 10^{-13}$ & $\cdots$ & $\cdots$ & $\mathrm{U}$ & $\ldots$ \\
\hline SC148 & J005151.6-731031 & $00^{\mathrm{h}} 51^{\mathrm{m}} 51^{\mathrm{s}} .56 /-73^{\circ} 10^{\prime} 31^{\prime \prime} .5$ & 7.4 & 30.8 & $7.3_{-0.9}^{+0.9} \times 10^{-13}$ & $1.90_{-0.40}^{+0.80}$ & SXP 172 & K & $\operatorname{BeXRB}(1,2)$ \\
\hline SC151 & J011535.0-731931 & $01^{\mathrm{h}} 15^{\mathrm{m}} 35^{\mathrm{s}} \cdot 00 /-73^{\circ} 19^{\prime} 31^{\prime \prime} 4$ & 5.3 & 1.6 & $3.4_{-1.6}^{+2.1} \times 10^{-13}$ & & $\ldots$ & $\mathrm{U}$ & $\ldots$ \\
\hline SC153 & J003351.0-730141 & $00^{\mathrm{h}} 33^{\mathrm{m}} 51^{\mathrm{s}} .05 /-73^{\circ} 01^{\prime} 41^{\prime \prime} \cdot 3$ & 6.1 & 2.1 & $1.4_{-0.5}^{+0.6} \times 10^{-14}$ & $3.70_{-2.20}^{+3.60}$ & $\ldots$ & $\mathrm{U}$ & $\ldots$ \\
\hline SC156 & J003418.4-733409 & $00^{\mathrm{h}} 34^{\mathrm{m}} 18^{\mathrm{s}} \cdot 42 /-73^{\circ} 34^{\prime} 09^{\prime \prime} \cdot 4$ & 6.1 & 3.1 & $7.5_{-1.2}^{+1.3} \times 10^{-13}$ & $1.40_{-1.30}^{+1.60}$ & $\cdots$ & $\mathrm{U}$ & $\ldots$ \\
\hline SC159 & J010137.0-720421 & $01^{\mathrm{h}} 01^{\mathrm{m}} 36^{\mathrm{s}} .97 /-72^{\circ} 04^{\prime} 21^{\prime \prime} 0$ & 6.4 & 7.3 & $3.3_{-0.7}^{+0.7} \times 10^{-13}$ & $2.08_{-0.59}^{+1.21}$ & $\begin{array}{c}\text { MQS J010137.52- } \\
720418.9\end{array}$ & K & $\begin{array}{c}\mathrm{AGN}(7, \\
8,10)\end{array}$ \\
\hline SC160 & J010151.2-722334 & $01^{\mathrm{h}} 01^{\mathrm{m}} 51^{\mathrm{s}} .19 /-72^{\circ} 23^{\prime} 34^{\prime \prime} 6$ & 5.0 & 21.7 & $1.1_{-0.1}^{+0.1} \times 10^{-12}$ & $1.30_{-0.40}^{+0.60}$ & SXP 175 & K & $\operatorname{BeXRB}(1,2)$ \\
\hline SC161 & J010029.2-722033 & $01^{\mathrm{h}} 00^{\mathrm{m}} 29^{\mathrm{s}} \cdot 17 /-72^{\circ} 20^{\prime} 33^{\prime \prime} \cdot 1$ & 5.3 & 11.4 & $2.7_{-0.6}^{+0.7} \times 10^{-13}$ & $1.82_{-0.68}^{+2.21}$ & [MA93] 1208 & K & $\operatorname{HMXB}(2,4)$ \\
\hline SC162 & J004243.9-740559 & $00^{\mathrm{h}} 42^{\mathrm{m}} 43^{\mathrm{s}} .93 /-74^{\circ} 05^{\prime} 59^{\prime \prime} 0$ & 5.2 & 4.8 & $9.2_{-2.8}^{+3.4} \times 10^{-14}$ & $\ldots$ & $\ldots$ & $\mathrm{U}$ & $\ldots$ \\
\hline SC163 & J012513.9-730356 & $01^{\mathrm{h}} 25^{\mathrm{m}} 13^{\mathrm{s}} \cdot 92 /-73^{\circ} 03^{\prime} 56^{\prime \prime} \cdot 2$ & 4.7 & 5.0 & $4.0_{-2.0}^{+2.4} \times 10^{-14}$ & $\ldots$ & $\ldots$ & $\mathrm{U}$ & $\ldots$ \\
\hline SC178 & J011102.8-715425 & $01^{\mathrm{h}} 11^{\mathrm{m}} 02^{\mathrm{s}} \cdot 82 /-71^{\circ} 54^{\prime} 25^{\prime \prime} \cdot 3$ & 5.9 & 0.0 & $3.3_{-1.4}^{+1.8} \times 10^{-13}$ & $\ldots$ & $\cdots$ & $\mathrm{U}$ & $\ldots$ \\
\hline SC182 & J010204.3-714132 & $01^{\mathrm{h}} 02^{\mathrm{m}} 04^{\mathrm{s}} \cdot 33 /-71^{\circ} 41^{\prime} 32^{\prime \prime} \cdot 2$ & 10.1 & 16.3 & $4.8_{-0.8}^{+0.9} \times 10^{-13}$ & $0.85_{-0.69}^{+1.35}$ & SXP 967 & $\mathrm{~K}$ & BeXRB (1) \\
\hline SC183 & J005431.4-721615 & $00^{\mathrm{h}} 54^{\mathrm{m}} 31^{\mathrm{s}} .35 /-72^{\circ} 16^{\prime} 15^{\prime \prime} 7$ & 5.9 & 2.0 & $4.7_{-1.8}^{+2.3} \times 10^{-14}$ & ... & $\ldots$ & $\mathrm{U}$ & $\ldots$ \\
\hline SC199 & J003026.9-731822 & $00^{\mathrm{h}} 30^{\mathrm{m}} 26^{\mathrm{s}} .88 /-73^{\circ} 18^{\prime} 22^{\prime \prime} 1$ & 5.7 & 12.3 & $3.7_{-0.5}^{+0.5} \times 10^{-13}$ & $1.80_{-0.30}^{+0.50}$ & $\cdots$ & $\mathrm{U}$ & \\
\hline
\end{tabular}


Table 2

(Continued)

\begin{tabular}{|c|c|c|c|c|c|c|c|c|c|}
\hline SC\# & $\begin{array}{c}\text { Name } \\
(1 \text { SCUBEDX J...) }\end{array}$ & $\begin{array}{l}\text { R.A./Decl. } \\
\text { (J2000) }\end{array}$ & $\begin{array}{l}\text { Err } \\
\left({ }^{\prime \prime}\right)\end{array}$ & $\begin{array}{l}\text { Det. } \\
(\%)\end{array}$ & $\begin{array}{r}f_{\text {mean }}(0.5-10 \mathrm{keV}) \\
\left(\mathrm{erg} \mathrm{s}^{-1} \mathrm{~cm}^{-2}\right)\end{array}$ & $\Gamma$ & Catalog Name & $\mathrm{Cl}$. & $\begin{array}{c}\text { Type } \\
\text { (Reference) }\end{array}$ \\
\hline$\overline{\mathrm{SC} 202}$ & J005718.2-722535 & $00^{\mathrm{h}} 57^{\mathrm{m}} 18^{\mathrm{s}} \cdot 18 /-72^{\circ} 25^{\prime} 35^{\prime \prime} 6$ & 5.3 & 2.2 & $3.2_{-0.7}^{+0.8} \times 10^{-13}$ & $1.60_{-1.10}^{+2.10}$ & $\begin{array}{c}\text { 1SXPS J005717.4- } \\
722538\end{array}$ & $\mathrm{~K}$ & $\ldots$ \\
\hline SC203 & J005251.6-721717 & $00^{\mathrm{h}} 52^{\mathrm{m}} 51^{\mathrm{s}} .60 /-72^{\circ} 17^{\prime} 17^{\prime \prime} .8$ & 5.2 & 19.5 & $5.9_{-1.1}^{+1.2} \times 10^{-13}$ & $1.29_{-0.85}^{+1.32}$ & SXP 327 & $\mathrm{~K}$ & $\operatorname{BeXRB}(1,2)$ \\
\hline SC204 & J005527.9-721100 & $00^{\mathrm{h}} 55^{\mathrm{m}} 27^{\mathrm{s}} \cdot 85 /-72^{\circ} 11^{\prime} 00^{\prime \prime} \cdot 9$ & 6.7 & 2.7 & $4.5_{-0.7}^{+0.8} \times 10^{-13}$ & $1.30_{-0.40}^{+0.80}$ & $\begin{array}{c}\text { 1SXPS J005527.4- } \\
721056\end{array}$ & K & $\cdots$ \\
\hline SC207 & J010055.3-712011 & $01^{\mathrm{h}} 00^{\mathrm{m}} 55^{\mathrm{s}} \cdot 32 /-71^{\circ} 20^{\prime} 11^{\prime \prime} 7$ & 7.0 & 5.2 & $1.7_{-0.3}^{+0.4} \times 10^{-13}$ & $3.50_{-1.60}^{+2.40}$ & $\begin{array}{c}\text { 3XMM J010055.5- } \\
712008\end{array}$ & $\mathrm{~K}$ & $\cdots$ \\
\hline SC213 & J003437.3-725655 & $00^{\mathrm{h}} 34^{\mathrm{m}} 37^{\mathrm{s}} .27 /-72^{\circ} 56^{\prime} 55^{\prime \prime} \cdot 2$ & 5.9 & 2.0 & $4.3_{-1.5}^{+1.8} \times 10^{-14}$ & $\cdots$ & $\ldots$ & $\mathrm{U}$ & $\cdots$ \\
\hline SC218 & J005322.2-722713 & $00^{\mathrm{h}} 53^{\mathrm{m}} 22^{\mathrm{s}} \cdot 22 /-72^{\circ} 27^{\prime} 13^{\prime \prime} \cdot 3$ & 7.3 & 14.9 & $1.1_{-0.2}^{+0.2} \times 10^{-12}$ & $0.86_{-0.44}^{+1.38}$ & SXP 138 & $\mathrm{~K}$ & BeXRB (1) \\
\hline SC226 & J005159.5-732919 & $00^{\mathrm{h}} 51^{\mathrm{m}} 59^{\mathrm{s}} .53 /-73^{\circ} 29^{\prime} 19^{\prime \prime} \cdot 2$ & 6.6 & 14.6 & $1.0_{-0.2}^{+0.2} \times 10^{-12}$ & $0.37_{-0.58}^{+1.09}$ & IGR J00515-7328 & $\mathrm{K}$ & $\operatorname{BeXRB}(6,2)$ \\
\hline SC227 & J005506.7-730545 & $00^{\mathrm{h}} 55^{\mathrm{m}} 06^{\mathrm{s}} .71 /-73^{\circ} 05^{\prime} 45^{\prime \prime} \cdot 9$ & 5.5 & 3.5 & $2.5_{-1.2}^{+1.6} \times 10^{-13}$ & $\ldots$ & $\cdots$ & $\mathrm{U}$ & $\ldots$ \\
\hline SC230 & J004537.6-720253 & $00^{\mathrm{h}} 45^{\mathrm{m}} 37^{\mathrm{s}} \cdot 57 /-72^{\circ} 02^{\prime} 53^{\prime \prime} \cdot 1$ & 5.7 & 5.1 & $2.1_{-0.5}^{+0.6} \times 10^{-13}$ & $1.50_{-1.50}^{+1.30}$ & $\ldots$ & $\mathrm{U}$ & $\ldots$ \\
\hline SC231 & J005735.6-721939 & $00^{\mathrm{h}} 57^{\mathrm{m}} 35^{\mathrm{s}} \cdot 62 /-72^{\circ} 19^{\prime} 39^{\prime \prime} 5$ & 6.7 & 4.4 & $5.6_{-1.0}^{+1.2} \times 10^{-13}$ & $0.79_{-0.72}^{+1.21}$ & SXP 565 & $\mathrm{~K}$ & $\operatorname{BeXRB}(1,2)$ \\
\hline SC233 & J010621.6-711710 & $01^{\mathrm{h}} 06^{\mathrm{m}} 21^{\mathrm{s}} \cdot 62 /-71^{\circ} 17^{\prime} 10^{\prime \prime} \cdot 7$ & 4.9 & 1.5 & $3.9_{-1.5}^{+1.7} \times 10^{-14}$ & $6.00_{-1.50}^{+18.50}$ & $\ldots$ & $\mathrm{U}$ & $\ldots$ \\
\hline SC234 & J010508.1-714403 & $01^{\mathrm{h}} 05^{\mathrm{m}} 08^{\mathrm{s}} \cdot 07 /-71^{\circ} 44^{\prime} 03^{\prime \prime} 4$ & 4.6 & 12.5 & $2.0_{-0.3}^{+0.3} \times 10^{-13}$ & $10.00_{-3.00}^{+64.00}$ & $\begin{array}{c}\text { 3XMM J010508.6- } \\
714358\end{array}$ & $\mathrm{~K}$ & $\ldots$ \\
\hline SC246 & J012121.8-721917 & $01^{\mathrm{h}} 21^{\mathrm{m}} 21^{\mathrm{s}} .78 /-72^{\circ} 19^{\prime} 17^{\prime \prime} \cdot 7$ & 5.5 & 9.3 & $1.5_{-0.4}^{+0.4} \times 10^{-13}$ & $\ldots$ & $\ldots$ & $\mathrm{U}$ & $\ldots$ \\
\hline SC249 & J011210.3-735421 & $01^{\mathrm{h}} 12^{\mathrm{m}} 10^{\mathrm{s}} \cdot 33 /-73^{\circ} 54^{\prime} 21^{\prime \prime} \cdot 1$ & 5.9 & 5.8 & $1.7_{-0.3}^{+0.4} \times 10^{-13}$ & $\ldots$ & $\ldots$ & $\mathrm{U}$ & $\cdots$ \\
\hline $\operatorname{SC} 250$ & J004648.4-733047 & $00^{\mathrm{h}} 46^{\mathrm{m}} 48^{\mathrm{s}} \cdot 40 /-73^{\circ} 30^{\prime} 47^{\prime \prime} \cdot 8$ & 5.1 & 4.4 & $4.4_{-2.0}^{+2.5} \times 10^{-14}$ & $\ldots$ & $\ldots$ & $\mathrm{U}$ & $\ldots$ \\
\hline SC251 & J003802.8-734458 & $00^{\mathrm{h}} 38^{\mathrm{m}} 02^{\mathrm{s}} \cdot 84 /-73^{\circ} 44^{\prime} 58^{\prime \prime} 0$ & 6.3 & 4.7 & $1.8_{-0.5}^{+0.6} \times 10^{-13}$ & $1.82_{-0.72}^{+1.42}$ & $\ldots$ & $\mathrm{U}$ & $\ldots$ \\
\hline SC255 & J012532.9-731602 & $01^{\mathrm{h}} 25^{\mathrm{m}} 32^{\mathrm{s}} .88 /-73^{\circ} 16^{\prime} 02^{\prime \prime} \cdot 7$ & 5.9 & 5.1 & $8.4_{-3.0}^{+3.7} \times 10^{-14}$ & $\ldots$ & 1WGA J0125.5-7315 & $\mathrm{K}$ & $\ldots$ \\
\hline SC256 & J013122.6-740044 & $01^{\mathrm{h}} 31^{\mathrm{m}} 22^{\mathrm{s}} \cdot 57 /-74^{\circ} 00^{\prime} 44^{\prime \prime} \cdot 4$ & 7.7 & 3.8 & $9.8_{-2.9}^{+3.5} \times 10^{-14}$ & $\ldots$ & $\cdots$ & $\mathrm{U}$ & $\ldots$ \\
\hline SC257 & J005241.1-724718 & $00^{\mathrm{h}} 52^{\mathrm{m}} 41^{\mathrm{s}} .09 /-72^{\circ} 47^{\prime} 18^{\prime \prime} 5$ & 6.5 & 2.1 & $1.1_{-0.2}^{+0.2} \times 10^{-13}$ & $0.93_{-0.48}^{+1.07}$ & $\begin{array}{c}\text { 1SXPS J005242.9- } \\
724721\end{array}$ & $\mathrm{~K}$ & $\cdots$ \\
\hline SC258 & J010312.1-710031 & $01^{\mathrm{h}} 03^{\mathrm{m}} 12^{\mathrm{s}} \cdot 10 /-71^{\circ} 00^{\prime} 31^{\prime \prime} 6$ & 5.3 & 5.9 & $7.0_{-2.6}^{+3.3} \times 10^{-13}$ & $\ldots$ & $\ldots$ & $\mathrm{U}$ & $\cdots$ \\
\hline SC259 & J005749.8-720235 & $00^{\mathrm{h}} 57^{\mathrm{m}} 49^{\mathrm{s}} .79 /-72^{\circ} 02^{\prime} 35^{\prime \prime} .1$ & 6.6 & 6.8 & $8.5_{-1.5}^{+1.7} \times 10^{-13}$ & $1.10_{-1.10}^{+1.40}$ & SXP 280 & K & $\operatorname{BeXRB}(1,2)$ \\
\hline SC264 & J003539.8-730904 & $00^{\mathrm{h}} 35^{\mathrm{m}} 39^{\mathrm{s}} .83 /-73^{\circ} 09^{\prime} 04^{\prime \prime} 9$ & 6.2 & 2.2 & $2.1_{-0.5}^{+0.6} \times 10^{-13}$ & $1.31_{-0.67}^{+1.54}$ & $\ldots$ & $\mathrm{U}$ & $\cdots$ \\
\hline SC266 & J011106.3-732854 & $01^{\mathrm{h}} 11^{\mathrm{m}} 06^{\mathrm{s}} \cdot 27 /-73^{\circ} 28^{\prime} 54^{\prime \prime} \cdot 9$ & 5.1 & 2.6 & $7.2_{-2.1}^{+2.6} \times 10^{-14}$ & $\ldots$ & $\ldots$ & $\mathrm{U}$ & $\ldots$ \\
\hline SC271 & J005354.9-722646 & $00^{\mathrm{h}} 53^{\mathrm{m}} 54^{\mathrm{s}} \cdot 94 /-72^{\circ} 26^{\prime} 46^{\prime \prime} 1$ & 4.4 & 23.5 & $2.8_{-0.2}^{+0.2} \times 10^{-12}$ & $1.10_{-0.21}^{+0.25}$ & SXP 46.6 & $\mathrm{~K}$ & $\operatorname{BeXRB}(1,2)$ \\
\hline SC276 & J003847.6-725244 & $00^{\mathrm{h}} 38^{\mathrm{m}} 47^{\mathrm{s}} \cdot 62 /-72^{\circ} 52^{\prime} 44^{\prime \prime} 8$ & 5.4 & 4.7 & $3.4_{-1.4}^{+1.8} \times 10^{-13}$ & $\cdots$ & $\cdots$ & $\mathrm{U}$ & $\cdots$ \\
\hline SC279 & J012013.9-724500 & $01^{\mathrm{h}} 20^{\mathrm{m}} 13^{\mathrm{s}} .93 /-72^{\circ} 45^{\prime} 00^{\prime \prime} 1$ & 5.1 & 12.2 & $3.0_{-0.4}^{+0.5} \times 10^{-13}$ & $9.40_{-2.80}^{+6.30}$ & HD 8353 & $\mathrm{~K}$ & Star (11) \\
\hline SC284 & J013102.7-741441 & $01^{\mathrm{h}} 31^{\mathrm{m}} 02^{\mathrm{s}} \cdot 68 /-74^{\circ} 14^{\prime} 41^{\prime \prime} 5$ & 6.0 & 11.1 & $2.2_{-0.5}^{+0.5} \times 10^{-13}$ & $10.00_{-8.00}^{+83.00}$ & $\begin{array}{c}\text { 1SXPS J013102.9- } \\
741435\end{array}$ & K & $\ldots$ \\
\hline SC285 & J012655.5-724410 & $01^{\mathrm{h}} 26^{\mathrm{m}} 55^{\mathrm{s}} .54 /-72^{\circ} 44^{\prime} 10^{\prime \prime} 8$ & 5.5 & 4.7 & $2.3_{-0.9}^{+1.1} \times 10^{-13}$ & $0.70_{-0.70}^{+4.10}$ & $\ldots$ & $\mathrm{U}$ & $\ldots$ \\
\hline SC287 & J012422.0-724248 & $01^{\mathrm{h}} 24^{\mathrm{m}} 21^{\mathrm{s}} \cdot 97 /-72^{\circ} 42^{\prime} 48^{\prime \prime} \cdot 7$ & 6.0 & 17.5 & $4.0_{-0.6}^{+0.7} \times 10^{-13}$ & & $\ldots$ & $\mathrm{U}$ & $\ldots$ \\
\hline SC288 & J012529.2-724258 & $01^{\mathrm{h}} 25^{\mathrm{m}} 29^{\mathrm{s}} .17 /-72^{\circ} 42^{\prime} 58^{\prime \prime} .5$ & 8.3 & 6.8 & $3.4_{-0.7}^{+0.8} \times 10^{-13}$ & $1.20_{-0.70}^{+1.00}$ & $\cdots$ & $\mathrm{U}$ & $\cdots$ \\
\hline SC300 & J010511.6-721158 & $01^{\mathrm{h}} 05^{\mathrm{m}} 11^{\mathrm{s}} \cdot 62 /-72^{\circ} 11^{\prime} 58^{\prime \prime} \cdot 4$ & 4.2 & 8.8 & $1.0_{-0.2}^{+0.2} \times 10^{-13}$ & $6.30_{-1.10}^{+2.20}$ & SXP 3.34 & K & $\operatorname{BeXRB}(1)$ \\
\hline SC301 & J012424.3-722902 & $01^{\mathrm{h}} 24^{\mathrm{m}} 24^{\mathrm{s}} .29 /-72^{\circ} 29^{\prime} 02^{\prime \prime} 7$ & 6.5 & 2.4 & $2.6_{-0.6}^{+0.7} \times 10^{-13}$ & $1.50_{-1.10}^{+2.70}$ & $\cdots$ & $\mathrm{U}$ & $\ldots$ \\
\hline SC303 & J010457.1-713704 & $01^{\mathrm{h}} 04^{\mathrm{m}} 57^{\mathrm{s}} .07 /-71^{\circ} 37^{\prime} 04^{\prime \prime} \cdot 7$ & 6.8 & 4.9 & $8.1_{-2.5}^{+3.1} \times 10^{-14}$ & $\ldots$ & $\ldots$ & $\mathrm{U}$ & $\ldots$ \\
\hline SC304 & J010102.6-713355 & $01^{\mathrm{h}} 01^{\mathrm{m}} 02^{\mathrm{s}} \cdot 57 /-71^{\circ} 33^{\prime} 55^{\prime \prime} .5$ & 5.3 & 3.6 & $4.8_{-2.5}^{+3.1} \times 10^{-14}$ & $\ldots$ & $\ldots$ & $\mathrm{U}$ & $\ldots$ \\
\hline SC306 & J005454.0-742700 & $00^{\mathrm{h}} 54^{\mathrm{m}} 54^{\mathrm{s}} .03 /-74^{\circ} 27^{\prime} 00^{\prime \prime} 7$ & 6.9 & 2.4 & $1.6_{-0.4}^{+0.5} \times 10^{-13}$ & $1.80_{-0.70}^{+1.00}$ & $\begin{array}{c}\text { 3XMM J005453.4- } \\
742705\end{array}$ & $\mathrm{~K}$ & $\ldots$ \\
\hline SC307 & J012055.7-733450 & $01^{\mathrm{h}} 20^{\mathrm{m}} 55^{\mathrm{s}} \cdot 72 /-73^{\circ} 34^{\prime} 50^{\prime \prime} 6$ & 6.0 & 8.3 & $4.4_{-0.7}^{+0.8} \times 10^{-13}$ & $1.80_{-0.70}^{+0.90}$ & $\begin{array}{c}\text { MQS J012056.05- } \\
733453.5\end{array}$ & $\mathrm{~K}$ & AGN (8) \\
\hline $\mathrm{SC} 312$ & J003738.0-722928 & $00^{\mathrm{h}} 37^{\mathrm{m}} 38^{\mathrm{s}} .00 /-72^{\circ} 29^{\prime} 28^{\prime \prime} 6$ & 5.9 & 5.3 & $2.3_{-0.6}^{+0.7} \times 10^{-13}$ & $1.49_{-0.76}^{+1.04}$ & 1WGA J0037.6-7229 & $\mathrm{K}$ & .. \\
\hline SC313 & J005732.6-721259 & $00^{\mathrm{h}} 57^{\mathrm{m}} 32^{\mathrm{s}} \cdot 60 /-72^{\circ} 12^{\prime} 59^{\prime \prime} \cdot 2$ & 5.1 & 2.8 & $1.7_{-0.3}^{+0.4} \times 10^{-13}$ & $2.39_{-0.62}^{+1.08}$ & $\begin{array}{c}\text { MQS J005732.75- } \\
721302.40\end{array}$ & $\mathrm{~K}$ & AGN (7) \\
\hline SC314 & J005017.3-723401 & $00^{\mathrm{h}} 50^{\mathrm{m}} 17^{\mathrm{s}} .28 /-72^{\circ} 34^{\prime} 01^{\prime \prime} .4$ & 4.8 & 2.5 & $7.5_{-2.8}^{+3.3} \times 10^{-14}$ & $\ldots$ & $\ldots$ & $\mathrm{U}$ & $\ldots$ \\
\hline SC317 & J005429.8-710334 & $00^{\mathrm{h}} 54^{\mathrm{m}} 29^{\mathrm{s}} .79 /-71^{\circ} 03^{\prime} 34^{\prime \prime} 6$ & 6.1 & 5.1 & $5.2_{-2.0}^{+2.5} \times 10^{-13}$ & $\ldots$ & $\ldots$ & $\mathrm{U}$ & $\ldots$ \\
\hline SC 320 & J011108.8-734528 & $01^{\mathrm{h}} 11^{\mathrm{m}} 08^{\mathrm{s}} .80 /-73^{\circ} 45^{\prime} 28^{\prime \prime} .0$ & 6.2 & 7.3 & $7.3_{-1.6}^{+1.8} \times 10^{-13}$ & $0.65_{-0.66}^{+1.46}$ & $\begin{array}{c}\text { MQS J011108.62- } \\
734529.1\end{array}$ & $\mathrm{U}$ & AGN (8) \\
\hline SC327 & J005727.1-732514 & $00^{\mathrm{h}} 57^{\mathrm{m}} 27^{\mathrm{s}} .09 /-73^{\circ} 25^{\prime} 14^{\prime \prime} \cdot 4$ & 6.3 & 19.0 & $1.8_{-0.2}^{+0.3} \times 10^{-12}$ & $1.10_{-1.50}^{+2.30}$ & SXP 101 & K & $\begin{array}{l}\operatorname{BeXRB}(1 \\
2,11)\end{array}$ \\
\hline SC333 & J012804.4-723913 & $01^{\mathrm{h}} 28^{\mathrm{m}} 04^{\mathrm{s}} \cdot 41 /-72^{\circ} 39^{\prime} 13^{\prime \prime} 0$ & 5.6 & 5.1 & $1.8_{-0.5}^{+0.6} \times 10^{-13}$ & $1.80_{-1.30}^{+1.40}$ & $\ldots$ & $\mathrm{U}$ & $\ldots$ \\
\hline SC334 & J002951.9-733145 & $00^{\mathrm{h}} 29^{\mathrm{m}} 51^{\mathrm{s}} .86 /-73^{\circ} 31^{\prime} 45^{\prime \prime} .5$ & 5.1 & 3.6 & $5.7_{-2.0}^{+2.6} \times 10^{-13}$ & $\ldots$ & $\ldots$ & $\mathrm{U}$ & $\cdots$ \\
\hline SC335 & J004943.1-732302 & $00^{\mathrm{h}} 49^{\mathrm{m}} 43^{\mathrm{s}} .14 /-73^{\circ} 23^{\prime} 02^{\prime \prime} 1$ & 6.2 & 5.6 & $9.7_{-1.5}^{+1.6} \times 10^{-13}$ & $0.12_{-0.58}^{+1.48}$ & SXP 756 & $\mathrm{~K}$ & BeXRB (1) \\
\hline SC336 & J012359.7-731620 & $01^{\mathrm{h}} 23^{\mathrm{m}} 59^{\mathrm{s}} \cdot 66 /-73^{\circ} 16^{\prime} 20^{\prime \prime} \cdot 9$ & 4.8 & 1.7 & $5.7_{-2.6}^{+3.1} \times 10^{-14}$ & $\ldots$ & $\ldots$ & $\mathrm{U}$ & $\ldots$ \\
\hline SC339 & J010836.1-722501 & $01^{\mathrm{h}} 08^{\mathrm{m}} 36^{\mathrm{s}} .09 /-72^{\circ} 25^{\prime} 01^{\prime \prime} 1$ & 6.0 & 10.0 & $2.5_{-0.6}^{+0.7} \times 10^{-13}$ & $1.80_{-0.66}^{+1.04}$ & $\begin{array}{c}\text { XMMU J010836.5- } \\
722459\end{array}$ & $\mathrm{~K}$ & AGN (11) \\
\hline
\end{tabular}


Table 2

(Continued)

\begin{tabular}{|c|c|c|c|c|c|c|c|c|c|}
\hline $\mathrm{SC} \#$ & $\begin{array}{c}\text { Name } \\
(1 \text { SCUBEDX J...) }\end{array}$ & $\begin{array}{l}\text { R.A./Decl. } \\
\text { (J2000) }\end{array}$ & $\begin{array}{l}\text { Err } \\
\left({ }^{\prime \prime}\right)\end{array}$ & $\begin{array}{l}\text { Det. } \\
(\%)\end{array}$ & $\begin{array}{r}f_{\text {mean }}(0.5-10 \mathrm{keV}) \\
\left(\mathrm{erg} \mathrm{s}^{-1} \mathrm{~cm}^{-2}\right)\end{array}$ & $\Gamma$ & Catalog Name & $\mathrm{Cl}$. & $\begin{array}{c}\text { Type } \\
\text { (Reference) }\end{array}$ \\
\hline$\overline{\mathrm{SC} 340}$ & J010305.0-724340 & $01^{\mathrm{h}} 03^{\mathrm{m}} 04^{\mathrm{s}} .97 /-72^{\circ} 43^{\prime} 40^{\prime \prime} 9$ & 4.9 & 6.0 & $1.4_{-0.4}^{+0.4} \times 10^{-13}$ & $\ldots$ & 2E 0101.3-7300 & $\mathrm{K}$ & $\ldots$ \\
\hline SC341 & J010459.3-721147 & $01^{\mathrm{h}} 04^{\mathrm{m}} 59^{\mathrm{s}} \cdot 30 /-72^{\circ} 11^{\prime} 47^{\prime \prime} 5$ & 6.0 & 6.2 & $8.5_{-2.5}^{+3.0} \times 10^{-14}$ & $\cdots$ & $\begin{array}{c}\text { 3XMM J010459.9- } \\
721148\end{array}$ & $\mathrm{~K}$ & $\cdots$ \\
\hline SC346 & J004338.1-721117 & $00^{\mathrm{h}} 43^{\mathrm{m}} 38^{\mathrm{s}} \cdot 09 /-72^{\circ} 11^{\prime} 17^{\prime \prime} 0$ & 5.1 & 4.2 & $1.1_{-0.3}^{+0.3} \times 10^{-13}$ & $\ldots$ & $\ldots$ & $\mathrm{U}$ & $\cdots$ \\
\hline SC349 & J004452.2-723729 & $00^{\mathrm{h}} 44^{\mathrm{m}} 52^{\mathrm{s}} \cdot 17 /-72^{\circ} 37^{\prime} 29^{\prime \prime} .^{2}$ & 5.2 & 4.5 & $6.4_{-2.0}^{+2.4} \times 10^{-14}$ & $\cdots$ & $\cdots$ & $\mathrm{U}$ & $\cdots$ \\
\hline SC351 & J005612.8-723328 & $00^{\mathrm{h}} 56^{\mathrm{m}} 12^{\mathrm{s}} .76 /-72^{\circ} 33^{\prime} 28^{\prime \prime} 4$ & 6.2 & 4.5 & $7.7_{-2.0}^{+2.4} \times 10^{-14}$ & $1.80_{-0.90}^{+3.70}$ & $\cdots$ & $\mathrm{U}$ & $\cdots$ \\
\hline SC353 & J004734.0-723014 & $00^{\mathrm{h}} 47^{\mathrm{m}} 33^{\mathrm{s}} \cdot 97 /-72^{\circ} 30^{\prime} 14^{\prime \prime} 1$ & 4.7 & 1.5 & $6.9_{-2.2}^{+2.6} \times 10^{-14}$ & $\ldots$ & $\cdots$ & $\mathrm{U}$ & $\cdots$ \\
\hline SC358 & J004947.3-713751 & $00^{\mathrm{h}} 49^{\mathrm{m}} 47^{\mathrm{s}} \cdot 28 /-71^{\circ} 37^{\prime} 51^{\prime \prime} 2$ & 7.0 & 5.0 & $1.2_{-0.3}^{+0.3} \times 10^{-13}$ & $\cdots$ & $\cdots$ & $\mathrm{U}$ & $\cdots$ \\
\hline SC364 & J003840.7-730809 & $00^{\mathrm{h}} 38^{\mathrm{m}} 40^{\mathrm{s}} \cdot 72 /-73^{\circ} 08^{\prime} 09^{\prime \prime} 9$ & 5.6 & 10.0 & $4.8_{-0.6}^{+0.7} \times 10^{-12}$ & $1.00_{-4.00}^{+3.00}$ & $\cdots$ & $\mathrm{U}$ & $\ldots$ \\
\hline SC366 & J010711.5-723534 & $01^{\mathrm{h}} 07^{\mathrm{m}} 11^{\mathrm{s}} .46 /-72^{\circ} 35^{\prime} 34^{\prime \prime} 1$ & 6.1 & 2.3 & $2.6_{-0.8}^{+1.0} \times 10^{-13}$ & $1.80_{-2.80}^{+4.40}$ & SXP 65.8 & $\mathrm{~K}$ & $\begin{array}{l}\operatorname{BeXRB}(1 \\
2,11)\end{array}$ \\
\hline SC369 & J012010.6-722818 & $01^{\mathrm{h}} 20^{\mathrm{m}} 10^{\mathrm{s}} \cdot 56 /-72^{\circ} 28^{\prime} 18^{\prime \prime} 8$ & 8.9 & 2.4 & $9.4_{-2.5}^{+3.0} \times 10^{-14}$ & $\cdots$ & $\begin{array}{l}\text { MQS J012010.56- } \\
\quad 722824.3\end{array}$ & $\mathrm{U}$ & AGN (8) \\
\hline SC372 & J005212.9-731916 & $00^{\mathrm{h}} 52^{\mathrm{m}} 12^{\mathrm{s}} \cdot 88 /-73^{\circ} 19^{\prime} 16^{\prime \prime} 7$ & 4.8 & 17.2 & $2.2_{-0.1}^{+0.1} \times 10^{-11}$ & $1.21_{-0.06}^{+0.12}$ & SXP 15.3 & $\mathrm{~K}$ & $\operatorname{BeXRB}(1,2)$ \\
\hline SC373 & J010053.0-724151 & $01^{\mathrm{h}} 00^{\mathrm{m}} 53^{\mathrm{s}} \cdot 02 /-72^{\circ} 41^{\prime} 51^{\prime \prime} 0$ & 6.2 & 12.8 & $1.9_{-0.3}^{+0.3} \times 10^{-13}$ & $11.00_{-1.30}^{+3.10}$ & $\begin{array}{l}\text { 1SXPS J010053.6- } \\
724149\end{array}$ & $\mathrm{~K}$ & $\cdots$ \\
\hline SC374 & J010435.6-722149 & $01^{\mathrm{h}} 04^{\mathrm{m}} 35^{\mathrm{s}} \cdot 57 /-72^{\circ} 21^{\prime} 49^{\prime \prime} 3$ & 8.2 & 10.0 & $1.1_{-0.2}^{+0.2} \times 10^{-12}$ & $0.81_{-0.98}^{+1.53}$ & [MA93] 1470 & $\mathrm{~K}$ & $\operatorname{HMXB}(2,4)$ \\
\hline SC376 & J005922.9-721013 & $00^{\mathrm{h}} 59^{\mathrm{m}} 22^{\mathrm{s}} \cdot 85 /-72^{\circ} 10^{\prime} 13^{\prime \prime} 9$ & 5.6 & 9.9 & $1.2_{-0.3}^{+0.4} \times 10^{-13}$ & . & SNR B0057-72.2 & $\mathrm{K}$ & SNR \\
\hline SC382 & J012106.8-725527 & $01^{\mathrm{h}} 21^{\mathrm{m}} 06^{\mathrm{s}} .81-72^{\circ} 55^{\prime} 27^{\prime \prime} 3$ & 5.9 & 8.3 & $2.7_{-0.7}^{+0.8} \times 10^{-13}$ & $0.86_{-0.84}^{+1.61}$ & $\begin{array}{l}\text { 3XMM J012106.7- } \\
725528\end{array}$ & $\mathrm{~K}$ & $\cdots$ \\
\hline SC384 & J012125.4-722728 & $01^{\mathrm{h}} 21^{\mathrm{m}} 25^{\mathrm{s}} \cdot 40 /-72^{\circ} 27^{\prime} 28^{\prime \prime} 7$ & 5.2 & 0.0 & $9.5_{-3.0}^{+3.6} \times 10^{-14}$ & $\ldots$ & $\ldots$ & $\mathrm{U}$ & $\cdots$ \\
\hline SC391 & J010015.6-742628 & $01^{\mathrm{h}} 00^{\mathrm{m}} 15^{\mathrm{s}} \cdot 62 /-74^{\circ} 26^{\prime} 28^{\prime \prime} 3$ & 6.9 & 4.9 & $1.6_{-0.4}^{+0.4} \times 10^{-13}$ & $\cdots$ & $\cdots$ & $\mathrm{U}$ & $\cdots$ \\
\hline SC395 & J010447.5-722258 & $01^{\mathrm{h}} 04^{\mathrm{m}} 47^{\mathrm{s}} \cdot 47 /-72^{\circ} 22^{\prime} 58^{\prime \prime} 0$ & 9.3 & 0.0 & $6.9_{-2.4}^{+3.0} \times 10^{-13}$ & $\ldots$ & $\begin{array}{l}\text { 3XMM J010447.4- } \\
722304\end{array}$ & $\mathrm{~K}$ & $\ldots$ \\
\hline SC396 & J003622.2-723621 & $00^{\mathrm{h}} 36^{\mathrm{m}} 22^{\mathrm{s}} \cdot 16 /-72^{\circ} 36^{\prime} 21^{\prime \prime} 0$ & 5.0 & 7.1 & $7.2_{-2.2}^{+2.6} \times 10^{-14}$ & $\ldots$ & $\ldots$ & $\mathrm{U}$ & $\ldots$ \\
\hline SC400 & J010620.4-720617 & $01^{\mathrm{h}} 06^{\mathrm{m}} 20^{\mathrm{s}} \cdot 35 /-72^{\circ} 06^{\prime} 17^{\prime \prime} 9$ & 11.1 & 0.0 & $1.6_{-0.8}^{+1.2} \times 10^{-13}$ & $\cdots$ & $\begin{array}{l}\text { 1SXPS J010615.7- } \\
\quad 720612\end{array}$ & $\mathrm{~K}$ & $\cdots$ \\
\hline SC401 & J010011.3-711818 & $01^{\mathrm{h}} 00^{\mathrm{m}} 11^{\mathrm{s}} \cdot 34 /-71^{\circ} 18^{\prime} 18^{\prime \prime} \cdot 9$ & 6.1 & 4.2 & $1.3_{-0.4}^{+0.5} \times 10^{-13}$ & $1.80_{-1.20}^{+1.70}$ & $\begin{array}{l}\text { 3XMM J010011.7- } \\
\quad 711821\end{array}$ & $\mathrm{~K}$ & $\cdots$ \\
\hline SC403 & J005456.4-722647 & $00^{\mathrm{h}} 54^{\mathrm{m}} 56^{\mathrm{s}} \cdot 43 /-72^{\circ} 26^{\prime} 47^{\prime \prime} 7$ & 4.0 & 20.0 & $5.9_{-0.3}^{+0.3} \times 10^{-12}$ & $1.08_{-0.09}^{+0.21}$ & SXP 59.0 & $\mathrm{~K}$ & $\operatorname{BeXRB}(1,2)$ \\
\hline SC404 & J004929.5-733107 & $00^{\mathrm{h}} 49^{\mathrm{m}} 29^{\mathrm{s}} .54 /-73^{\circ} 31^{\prime} 07^{\prime \prime} 8$ & 6.7 & 4.5 & $9.7_{-1.7}^{+1.8} \times 10^{-13}$ & $0.40_{-0.50}^{+0.70}$ & [MA93] 302 & $\mathrm{~K}$ & $\operatorname{HMXB}(2,4)$ \\
\hline SC419 & J004547.9-735329 & $00^{\mathrm{h}} 45^{\mathrm{m}} 47^{\mathrm{s}} .87 /-73^{\circ} 53^{\prime} 29^{\prime \prime} 4$ & 5.6 & 2.4 & $4.0_{-1.1}^{+1.3} \times 10^{-13}$ & $0.76_{-0.91}^{+1.34}$ & 1WGA J0045.7-7353 & $\mathrm{K}$ & $\cdots$ \\
\hline $\mathrm{SC} 423$ & J005553.0-730506 & $00^{\mathrm{h}} 55^{\mathrm{m}} 53^{\mathrm{s}} .01 /-73^{\circ} 05^{\prime} 06^{\prime \prime} 9$ & 4.8 & 1.4 & $7.7_{-2.4}^{+2.7} \times 10^{-14}$ & $\ldots$ & $\ldots$ & $\mathrm{U}$ & $\cdots$ \\
\hline SC425 & J004604.8-725300 & $00^{\mathrm{h}} 46^{\mathrm{m}} 04^{\mathrm{s}} .83 /-72^{\circ} 53^{\prime} 00^{\prime \prime} 8$ & 8.9 & 4.9 & $4.4_{-1.7}^{+2.1} \times 10^{-14}$ & $\ldots$ & $\ldots$ & $\mathrm{U}$ & $\cdots$ \\
\hline SC428 & J010102.4-720656 & $01^{\mathrm{h}} 01^{\mathrm{m}} 02^{\mathrm{s}} \cdot 44 /-72^{\circ} 06^{\prime} 56^{\prime \prime} 8$ & 5.5 & 9.5 & $5.8_{-1.2}^{+1.4} \times 10^{-13}$ & $0.60_{-0.54}^{+1.14}$ & SXP 304 & $\mathrm{~K}$ & $\operatorname{BeXRB}(1,2)$ \\
\hline SC430 & J005708.8-724202 & $00^{\mathrm{h}} 57^{\mathrm{m}} 08^{\mathrm{s}} .81 /-72^{\circ} 42^{\prime} 02^{\prime \prime} 4$ & 4.9 & 2.4 & $3.7_{-1.6}^{+2.0} \times 10^{-13}$ & $\ldots$ & $\cdots$ & $\mathrm{U}$ & $\cdots$ \\
\hline SC431 & J010109.5-720740 & $01^{\mathrm{h}} 01^{\mathrm{m}} 09^{\mathrm{s}} .50 /-72^{\circ} 07^{\prime} 40^{\prime \prime} \cdot 7$ & 4.3 & 4.9 & $5.8_{-2.6}^{+3.1} \times 10^{-14}$ & $\cdots$ & $\cdots$ & $\mathrm{U}$ & $\cdots$ \\
\hline SC432 & J005655.0-720643 & $00^{\mathrm{h}} 56^{\mathrm{m}} 54^{\mathrm{s}} .97 /-72^{\circ} 06^{\prime} 43^{\prime \prime} 7$ & 4.7 & 4.3 & $1.1_{-0.3}^{+0.3} \times 10^{-13}$ & $\cdots$ & $\begin{array}{l}\text { 1SXPS J005656.5- } \\
\quad 720646\end{array}$ & $\mathrm{~K}$ & $\cdots$ \\
\hline SC433 & J010740.1-710022 & $01^{\mathrm{h}} 07^{\mathrm{m}} 40^{\mathrm{s}} \cdot 14 /-71^{\circ} 00^{\prime} 22^{\prime \prime} 2$ & 5.6 & 3.1 & $4.2_{-1.8}^{+2.4} \times 10^{-13}$ & $\ldots$ & $\ldots$ & $\mathrm{U}$ & $\cdots$ \\
\hline SC435 & J012849.6-741921 & $01^{\mathrm{h}} 28^{\mathrm{m}} 49^{\mathrm{s}} \cdot 61 /-74^{\circ} 19^{\prime} 21^{\prime \prime} 6$ & 6.4 & 5.9 & $1.3_{-0.4}^{+0.5} \times 10^{-13}$ & $\ldots$ & $\cdots$ & $\mathrm{U}$ & $\cdots$ \\
\hline SC437 & J012721.2-724636 & $01^{\mathrm{h}} 27^{\mathrm{m}} 21^{\mathrm{s}} \cdot 17 /-72^{\circ} 46^{\prime} 36^{\prime \prime} 3$ & 7.1 & 2.4 & $7.8_{-2.8}^{+3.5} \times 10^{-14}$ & $5.00_{-4.00}^{+7.00}$ & $\cdots$ & $\mathrm{U}$ & $\cdots$ \\
\hline SC441 & J013358.3-735226 & $01^{\mathrm{h}} 33^{\mathrm{m}} 58^{\mathrm{s}} \cdot 27 /-73^{\circ} 52^{\prime} 26^{\prime \prime} 7$ & 6.7 & 3.1 & $1.2_{-0.4}^{+0.4} \times 10^{-13}$ & $2.40_{-1.00}^{+1.10}$ & $\ldots$ & $\mathrm{U}$ & $\cdots$ \\
\hline SC444 & J004950.8-730755 & $00^{\mathrm{h}} 49^{\mathrm{m}} 50^{\mathrm{s}} \cdot 77 /-73^{\circ} 07^{\prime} 55^{\prime \prime} 6$ & 6.1 & 2.4 & $5.1_{-2.1}^{+2.7} \times 10^{-13}$ & $\cdots$ & $\cdots$ & $\mathrm{U}$ & $\cdots$ \\
\hline SC448 & J011526.4-722958 & $01^{\mathrm{h}} 15^{\mathrm{m}} 26^{\mathrm{s}} \cdot 44 /-72^{\circ} 29^{\prime} 58^{\prime \prime} \cdot 9$ & 5.5 & 4.8 & $5.1_{-1.0}^{+1.1} \times 10^{-13}$ & $3.00_{-2.50}^{+3.50}$ & $\begin{array}{c}\text { MQS J011526.28- } \\
722958.5\end{array}$ & $\mathrm{~K}$ & $\operatorname{AGN}(8,11)$ \\
\hline $\mathrm{SC} 454$ & J005103.9-721203 & $00^{\mathrm{h}} 51^{\mathrm{m}} 03^{\mathrm{s}} .87 /-72^{\circ} 12^{\prime} 03^{\prime \prime} 2$ & 4.6 & 4.5 & $1.1_{-0.3}^{+0.3} \times 10^{-13}$ & $\ldots$ & $\ldots$ & $\mathrm{U}$ & $\cdots$ \\
\hline SC455 & J004903.8-723136 & $00^{\mathrm{h}} 49^{\mathrm{m}} 03.75 /-72^{\circ} 31^{\prime} 36^{\prime \prime} 6$ & 5.0 & 3.1 & $3.6_{-1.6}^{+2.0} \times 10^{-13}$ & $\ldots$ & $\cdots$ & $\mathrm{U}$ & $\cdots$ \\
\hline SC459 & J003429.5-732035 & $00^{\mathrm{h}} 34^{\mathrm{m}} 29^{\mathrm{s}} \cdot 47 /-73^{\circ} 20^{\prime} 35^{\prime \prime} 5$ & 4.9 & 1.4 & $5.4_{-1.9}^{+2.3} \times 10^{-14}$ & $\cdots$ & $\ldots$ & $\mathrm{U}$ & $\ldots$ \\
\hline SC461 & J011728.6-731146 & $01^{\mathrm{h}} 17^{\mathrm{m}} 28^{\mathrm{s}} \cdot 55 /-73^{\circ} 11^{\prime} 46^{\prime \prime} 1$ & 5.7 & 2.4 & $1.2_{-0.3}^{+0.4} \times 10^{-13}$ & $\ldots$ & $\begin{array}{c}\text { 3XMM J011728.4- } \\
731144\end{array}$ & $\mathrm{~K}$ & $\ldots$ \\
\hline SC466 & J005910.9-723002 & $00^{\mathrm{h}} 59^{\mathrm{m}} 10^{\mathrm{s}} .91 /-72^{\circ} 30^{\prime} 02^{\prime \prime} 0$ & 4.7 & 1.6 & $5.7_{-2.2}^{+2.6} \times 10^{-14}$ & $\ldots$ & $\ldots$ & $\mathrm{U}$ & $\ldots$ \\
\hline SC467 & J004306.2-721924 & $00^{\mathrm{h}} 43^{\mathrm{m}} 06^{\mathrm{s}} \cdot 16 /-72^{\circ} 19^{\prime} 24^{\prime \prime} 5$ & 4.4 & 7.0 & $7.1_{-1.6}^{+1.8} \times 10^{-14}$ & $5.00_{-0.99}^{+2.17}$ & $\ldots$ & $\mathrm{U}$ & $\cdots$ \\
\hline SC468 & J002953.6-730657 & $00^{\mathrm{h}} 29^{\mathrm{m}} 53^{\mathrm{s}} \cdot 62 /-73^{\circ} 06^{\prime} 57^{\prime \prime} 3$ & 5.2 & 3.4 & $2.3_{-0.9}^{+1.1} \times 10^{-13}$ & $\ldots$ & $\ldots$ & $\mathrm{U}$ & $\cdots$ \\
\hline SC469 & J011001.0-731526 & $01^{\mathrm{h}} 10^{\mathrm{m}} 01^{\mathrm{s}} .01 /-73^{\circ} 15^{\prime} 26^{\prime \prime} 5$ & 5.5 & 2.6 & $4.8_{-2.3}^{+2.9} \times 10^{-13}$ & $\cdots$ & $\ldots$ & $\mathrm{U}$ & $\cdots$ \\
\hline SC470 & J004944.9-743118 & $00^{\mathrm{h}} 49^{\mathrm{m}} 44^{\mathrm{s}} \cdot 94 /-74^{\circ} 31^{\prime} 18^{\prime \prime} 9$ & 5.1 & 2.5 & $4.6_{-1.5}^{+1.8} \times 10^{-14}$ & $\ldots$ & $\ldots$ & $\mathrm{U}$ & $\ldots$ \\
\hline SC473 & J003007.0-740012 & $00^{\mathrm{h}} 30^{\mathrm{m}} 07^{\mathrm{s}} \cdot 04 /-74^{\circ} 00^{\prime} 12^{\prime \prime} \cdot 7$ & 5.6 & 5.0 & $2.5_{-0.5}^{+0.6} \times 10^{-13}$ & $\ldots$ & $\begin{array}{l}\text { 1RXS J003009.0- } \\
\quad 740009\end{array}$ & $\mathrm{~K}$ & $\ldots$ \\
\hline SC475 & J012250.9-731233 & $01^{\mathrm{h}} 22^{\mathrm{m}} 50^{\mathrm{s}} \cdot 89 /-73^{\circ} 12^{\prime} 33^{\prime \prime} 8$ & 5.1 & 4.8 & $7.7_{-2.8}^{+3.5} \times 10^{-13}$ & $\cdots$ & $\cdots$ & $\mathrm{U}$ & $\cdots$ \\
\hline
\end{tabular}


Table 2

(Continued)

\begin{tabular}{|c|c|c|c|c|c|c|c|c|c|}
\hline SC\# & $\begin{array}{c}\text { Name } \\
(1 \text { SCUBEDX J...) }\end{array}$ & $\begin{array}{l}\text { R.A./Decl. } \\
\text { (J2000) }\end{array}$ & $\begin{array}{l}\text { Err } \\
\left({ }^{\prime \prime}\right)\end{array}$ & $\begin{array}{l}\text { Det. } \\
(\%)\end{array}$ & $\begin{array}{r}f_{\text {mean }}(0.5-10 \mathrm{keV}) \\
\quad\left(\mathrm{erg} \mathrm{s}^{-1} \mathrm{~cm}^{-2}\right)\end{array}$ & $\Gamma$ & Catalog Name & $\mathrm{Cl}$. & $\begin{array}{c}\text { Type } \\
\text { (Reference) }\end{array}$ \\
\hline$\overline{\mathrm{SC} 480}$ & J010634.6-731916 & $01^{\mathrm{h}} 06^{\mathrm{m}} 34^{\mathrm{s}} \cdot 63 /-73^{\circ} 19^{\prime} 16^{\prime \prime} 3$ & 4.9 & 3.8 & $3.6_{-1.8}^{+2.2} \times 10^{-13}$ & $\ldots$ & $\ldots$ & $\mathrm{U}$ & $\ldots$ \\
\hline SC482 & J010331.1-730141 & $01^{\mathrm{h}} 03^{\mathrm{m}} 31^{\mathrm{s}} \cdot 10 /-73^{\circ} 01^{\prime} 41^{\prime \prime} \cdot 2$ & 7.5 & 7.9 & $3.2_{-0.6}^{+0.7} \times 10^{-13}$ & $1.26_{-0.71}^{+1.86}$ & [M2002] SMC 56587 & K & $\operatorname{HMXB}(2,5)$ \\
\hline SC483 & J005559.4-733746 & $00^{\mathrm{h}} 55^{\mathrm{m}} 59^{\mathrm{s}} .37 /-73^{\circ} 37^{\prime} 46^{\prime \prime} \cdot 7$ & 4.8 & 3.8 & $9.4_{-2.9}^{+3.4} \times 10^{-14}$ & $\ldots$ & $\ldots$ & $\mathrm{U}$ & $\ldots$ \\
\hline SC487 & J005706.6-722641 & $00^{\mathrm{h}} 57^{\mathrm{m}} 06^{\mathrm{s}} \cdot 58 /-72^{\circ} 26^{\prime} 41^{\prime \prime} 6$ & 5.4 & 4.1 & $4.6_{-1.9}^{+2.4} \times 10^{-13}$ & $\ldots$ & 1WGA J0057.3-7226 & $\mathrm{K}$ & $\ldots$ \\
\hline SC489 & J004611.6-715345 & $00^{\mathrm{h}} 46^{\mathrm{m}} 11^{\mathrm{s}} .57 /-71^{\circ} 53^{\prime} 45^{\prime \prime} 5$ & 5.0 & 3.1 & $8.4_{-2.6}^{+3.0} \times 10^{-14}$ & $\cdots$ & $\cdots$ & $\mathrm{U}$ & $\ldots$ \\
\hline SC493 & J010732.7-714549 & $01^{\mathrm{h}} 07^{\mathrm{m}} 32^{\mathrm{s}} \cdot 68 /-71^{\circ} 45^{\prime} 49^{\prime \prime} 5$ & 6.1 & 0.0 & $1.4_{-0.5}^{+0.6} \times 10^{-12}$ & $2.10_{-2.60}^{+8.80}$ & $\ldots$ & $\mathrm{U}$ & $\ldots$ \\
\hline SC501 & J003302.8-742335 & $00^{\mathrm{h}} 33^{\mathrm{m}} 02^{\mathrm{s}} \cdot 81 /-74^{\circ} 23^{\prime} 35^{\prime \prime} .0$ & 8.1 & 2.9 & $6.0_{-2.2}^{+2.8} \times 10^{-13}$ & $\ldots$ & $\ldots$ & $\mathrm{U}$ & $\ldots$ \\
\hline SC502 & J003524.0-733216 & $00^{\mathrm{h}} 35^{\mathrm{m}} 23^{\mathrm{s}} \cdot 97 /-73^{\circ} 32^{\prime} 16^{\prime \prime} 7$ & 6.4 & 2.4 & $1.6_{-0.4}^{+0.4} \times 10^{-13}$ & $\ldots$ & 1WGA J0035.3-7332 & $\mathrm{K}$ & $\ldots$ \\
\hline SC509 & J013657.8-732641 & $01^{\mathrm{h}} 36^{\mathrm{m}} 57^{\mathrm{s}} \cdot 76 /-73^{\circ} 26^{\prime} 41^{\prime \prime} \cdot 1$ & 6.4 & 3.0 & $9.1_{-3.1}^{+3.9} \times 10^{-13}$ & $\ldots$ & $\ldots$ & $\mathrm{U}$ & $\ldots$ \\
\hline SC520 & J004820.0-733151 & $00^{\mathrm{h}} 48^{\mathrm{m}} 20^{\mathrm{s}} \cdot 03 /-73^{\circ} 31^{\prime} 51^{\prime \prime} 1$ & 5.7 & 14.6 & $4.3_{-0.6}^{+0.7} \times 10^{-13}$ & $\ldots$ & SMC Symbiotic star 3 & $\mathrm{~K}$ & $\begin{array}{l}\text { Symbiotic } \\
\text { Star (13) }\end{array}$ \\
\hline SC526 & J005444.9-722527 & $00^{\mathrm{h}} 54^{\mathrm{m}} 44^{\mathrm{s}} \cdot 92 /-72^{\circ} 25^{\prime} 27^{\prime \prime} \cdot 9$ & 5.5 & 2.0 & $3.3_{-0.9}^{+1.1} \times 10^{-13}$ & $0.40_{-1.20}^{+2.30}$ & SXP 6.62 & $\mathrm{~K}$ & $\operatorname{BeXRB}(1)$ \\
\hline SC527 & J005431.4-721809 & $00^{\mathrm{h}} 54^{\mathrm{m}} 31^{\mathrm{s}} .39 /-72^{\circ} 18^{\prime} 09^{\prime \prime} .8$ & 5.6 & 2.0 & $8.2_{-2.3}^{+2.7} \times 10^{-14}$ & $2.80_{-2.20}^{+5.20}$ & $\begin{array}{l}\text { 1SXPS J005431.1- } \\
721808\end{array}$ & $\mathrm{~K}$ & $\ldots$ \\
\hline SC546 & J011854.0-731433 & $01^{\mathrm{h}} 18^{\mathrm{m}} 53^{\mathrm{s}} .99 /-73^{\circ} 14^{\prime} 33^{\prime \prime}{ }^{\prime}$ & 4.5 & 2.6 & $1.1_{-0.3}^{+0.4} \times 10^{-13}$ & $\ldots$ & $\ldots$ & $\mathrm{U}$ & $\ldots$ \\
\hline SC552 & J010132.8-713258 & $01^{\mathrm{h}} 01^{\mathrm{m}} 32^{\mathrm{s}} \cdot 76 /-71^{\circ} 32^{\prime} 58^{\prime \prime} \cdot 6$ & 5.6 & 2.4 & $2.0_{-0.4}^{+0.5} \times 10^{-13}$ & $\cdots$ & $\begin{array}{l}\text { 3XMM J010132.2- } \\
713249\end{array}$ & $\mathrm{~K}$ & $\ldots$ \\
\hline SC554 & J005749.8-711802 & $00^{\mathrm{h}} 57^{\mathrm{m}} 49^{\mathrm{s}} \cdot 77 /-71^{\circ} 18^{\prime} 02^{\prime \prime} \cdot 7$ & 6.3 & 5.3 & $1.9_{-0.4}^{+0.4} \times 10^{-13}$ & $1.85_{-0.69}^{+1.03}$ & $\begin{array}{l}\text { 3XMM J005749.6- } \\
711802\end{array}$ & K & $\ldots$ \\
\hline SC565 & J004956.2-743204 & $00^{\mathrm{h}} 49^{\mathrm{m}} 56^{\mathrm{s}} \cdot 21 /-74^{\circ} 32^{\prime} 04^{\prime \prime} 1$ & 5.6 & 0.0 & $5.1_{-1.5}^{+1.8} \times 10^{-13}$ & $0.40_{-1.30}^{+2.20}$ & $\begin{array}{c}\text { MQS J004955.43- } \\
743200.9\end{array}$ & $\mathrm{~K}$ & AGN (8) \\
\hline SC567 & J011411.6-735414 & $01^{\mathrm{h}} 14^{\mathrm{m}} 11^{\mathrm{s}} \cdot 62 /-73^{\circ} 54^{\prime} 14^{\prime \prime} .5$ & 5.5 & 3.6 & $9.1_{-2.8}^{+3.4} \times 10^{-14}$ & $\ldots$ & $\ldots$ & $\mathrm{U}$ & $\ldots$ \\
\hline SC569 & J005111.6-732059 & $00^{\mathrm{h}} 51^{\mathrm{m}} 11^{\mathrm{s}} .61 /-73^{\circ} 20^{\prime} 59^{\prime \prime} 8$ & 7.0 & 5.8 & $1.1_{-0.3}^{+0.4} \times 10^{-13}$ & $\ldots$ & $\begin{array}{l}\text { 3XMM J005112.1- } \\
732056\end{array}$ & $\mathrm{~K}$ & $\ldots$ \\
\hline SC579 & J004545.5-724147 & $00^{\mathrm{h}} 45^{\mathrm{m}} 45^{\mathrm{s}} .53 /-72^{\circ} 41^{\prime} 47^{\prime \prime} .5$ & 6.6 & 2.6 & $2.4_{-1.2}^{+1.6} \times 10^{-13}$ & $\ldots$ & $\ldots$ & $\mathrm{U}$ & $\ldots$ \\
\hline SC583 & J010009.0-730726 & $01^{\mathrm{h}} 00^{\mathrm{m}} 09^{\mathrm{s}} .03 /-73^{\circ} 07^{\prime} 26^{\prime \prime} 3$ & 11.8 & 0.0 & $4.8_{-1.9}^{+2.5} \times 10^{-13}$ & $\ldots$ & $\begin{array}{l}\text { 1SXPS J010014.1- } \\
730722\end{array}$ & $\mathrm{~K}$ & $\ldots$ \\
\hline SC596 & J005723.9-725003 & $00^{\mathrm{h}} 57^{\mathrm{m}} 23^{\mathrm{s}} \cdot 90 /-72^{\circ} 50^{\prime} 03^{\prime \prime} .4$ & 6.4 & 6.5 & $9.2_{-2.9}^{+3.5} \times 10^{-14}$ & $\cdots$ & $\begin{array}{c}\text { 1SXPS J005722.5- } \\
\quad 725011\end{array}$ & $\mathrm{~K}$ & $\cdots$ \\
\hline SC597 & J012735.5-724943 & $01^{\mathrm{h}} 27^{\mathrm{m}} 35^{\mathrm{s}} \cdot 54 /-72^{\circ} 49^{\prime} 43^{\prime \prime} 6$ & 5.2 & 4.7 & $1.0_{-0.3}^{+0.4} \times 10^{-13}$ & $\ldots$ & $\ldots$ & $\mathrm{U}$ & $\ldots$ \\
\hline SC600 & J011450.8-715629 & $01^{\mathrm{h}} 14^{\mathrm{m}} 50^{\mathrm{s}} .80 /-71^{\circ} 56^{\prime} 29^{\prime \prime} \cdot 3$ & 4.3 & 2.4 & $6.8_{-2.4}^{+2.8} \times 10^{-14}$ & $\ldots$ & $\cdots$ & $\mathrm{U}$ & $\ldots$ \\
\hline SC603 & J011526.2-715254 & $01^{\mathrm{h}} 15^{\mathrm{m}} 26^{\mathrm{s}} .22 /-71^{\circ} 52^{\prime} 54^{\prime \prime} \cdot 2$ & 5.8 & 2.4 & $4.2_{-1.9}^{+2.4} \times 10^{-13}$ & $\ldots$ & $\ldots$ & $\mathrm{U}$ & $\ldots$ \\
\hline SC608 & J005747.3-715943 & $00^{\mathrm{h}} 57^{\mathrm{m}} 47^{\mathrm{s}} .26 /-71^{\circ} 59^{\prime} 43^{\prime \prime} .4$ & 4.5 & 2.3 & $7.3_{-2.8}^{+3.3} \times 10^{-14}$ & $\ldots$ & $\ldots$ & $\mathrm{U}$ & $\ldots$ \\
\hline SC611 & J005914.4-721435 & $00^{\mathrm{h}} 59^{\mathrm{m}} 14^{\mathrm{s}} \cdot 42 /-72^{\circ} 14^{\prime} 35^{\prime \prime} 6$ & 6.0 & 5.3 & $1.3_{-0.3}^{+0.4} \times 10^{-13}$ & $\ldots$ & $\cdots$ & $\mathrm{U}$ & $\ldots$ \\
\hline SC613 & J010106.5-721254 & $01^{\mathrm{h}} 01^{\mathrm{m}} 06^{\mathrm{s}} .51 /-72^{\circ} 12^{\prime} 54^{\prime \prime} 6$ & 5.4 & 1.7 & $6.7_{-2.4}^{+2.9} \times 10^{-14}$ & $\ldots$ & $\cdots$ & $\mathrm{U}$ & $\ldots$ \\
\hline SC618 & J004351.2-715508 & $00^{\mathrm{h}} 43^{\mathrm{m}} 51^{\mathrm{s}} .25 /-71^{\circ} 55^{\prime} 08^{\prime \prime} .5$ & 6.6 & 5.8 & $1.4_{-0.4}^{+0.4} \times 10^{-13}$ & $\ldots$ & $\ldots$ & $\mathrm{U}$ & $\cdots$ \\
\hline SC619 & J004521.2-713434 & $00^{\mathrm{h}} 45^{\mathrm{m}} 21^{\mathrm{s}} .25 /-71^{\circ} 34^{\prime} 34^{\prime \prime} \cdot 9$ & 5.1 & 1.5 & $7.1_{-2.5}^{+3.0} \times 10^{-14}$ & $\ldots$ & $\ldots$ & $\mathrm{U}$ & $\ldots$ \\
\hline SC621 & J005613.6-715449 & $00^{\mathrm{h}} 56^{\mathrm{m}} 13^{\mathrm{s}} \cdot 55 /-71^{\circ} 54^{\prime} 49^{\prime \prime} \cdot 1$ & 5.0 & 2.9 & $5.4_{-1.4}^{+1.6} \times 10^{-14}$ & $17.60_{-20.60}^{+81.30}$ & $\ldots$ & $\mathrm{U}$ & $\ldots$ \\
\hline SC629 & J005722.6-715904 & $00^{\mathrm{h}} 57^{\mathrm{m}} 22^{\mathrm{s}} \cdot 63 /-71^{\circ} 59^{\prime} 04^{\prime \prime} 5$ & 5.1 & 0.0 & $5.3_{-2.2}^{+2.8} \times 10^{-13}$ & $\ldots$ & $\ldots$ & $\mathrm{U}$ & $\ldots$ \\
\hline SC630 & J005936.2-715455 & $00^{\mathrm{h}} 59^{\mathrm{m}} 36^{\mathrm{s}} .21 /-71^{\circ} 54^{\prime} 55^{\prime \prime} 6$ & 4.5 & 1.4 & $8.7_{-3.2}^{+3.6} \times 10^{-14}$ & $\ldots$ & & K & $\ldots$ \\
\hline SC639 & J010816.4-714822 & $01^{\mathrm{h}} 08^{\mathrm{m}} 16^{\mathrm{s}} \cdot 40 /-71^{\circ} 48^{\prime} 22^{\prime \prime} \cdot 2$ & 8.1 & 5.1 & $7.6_{-2.7}^{+3.4} \times 10^{-13}$ & $\ldots$ & $\ldots$ & $\mathrm{U}$ & $\ldots$ \\
\hline SC641 & J010849.2-720553 & $01^{\mathrm{h}} 08^{\mathrm{m}} 49^{\mathrm{s}} .22 /-72^{\circ} 05^{\prime} 53^{\prime \prime} .4$ & 4.9 & 2.6 & $9.2_{-3.4}^{+4.1} \times 10^{-14}$ & $\ldots$ & $\begin{array}{l}\text { 3XMM J010848.6- } \\
720547\end{array}$ & $\mathrm{~K}$ & $\ldots$ \\
\hline SC645 & J004533.8-720641 & $00^{\mathrm{h}} 45^{\mathrm{m}} 33^{\mathrm{s}} .78 /-72^{\circ} 06^{\prime} 41^{\prime \prime} 3$ & 4.7 & 2.4 & $1.3_{-0.3}^{+0.4} \times 10^{-13}$ & $\ldots$ & $\ldots$ & $\mathrm{U}$ & $\ldots$ \\
\hline SC658 & J005954.1-720128 & $00^{\mathrm{h}} 59^{\mathrm{m}} 54^{\mathrm{s}} .12 /-72^{\circ} 01^{\prime} 28^{\prime \prime} \cdot 9$ & 5.3 & 0.0 & $3.7_{-1.7}^{+2.1} \times 10^{-14}$ & $\ldots$ & $\ldots$ & $\mathrm{U}$ & $\ldots$ \\
\hline SC670 & J004812.7-732153 & $00^{\mathrm{h}} 48^{\mathrm{m}} 12^{\mathrm{s}} \cdot 69 /-73^{\circ} 21^{\prime} 53^{\prime \prime} \cdot 3$ & 4.9 & 3.2 & $9.0_{-2.9}^{+3.4} \times 10^{-14}$ & $\ldots$ & SXP 11.9 & $\mathrm{~K}$ & BeXRB (1) \\
\hline SC677 & J005820.3-721753 & $00^{\mathrm{h}} 58^{\mathrm{m}} 20^{\mathrm{s}} \cdot 28 /-72^{\circ} 17^{\prime} 53^{\prime \prime} 6$ & 9.9 & 4.0 & $1.9_{-0.9}^{+1.1} \times 10^{-13}$ & $\ldots$ & $\begin{array}{c}\text { 1SXPS J005816.6- } \\
721801\end{array}$ & $\mathrm{~K}$ & $\ldots$ \\
\hline SC689 & J005640.5-715803 & $00^{\mathrm{h}} 56^{\mathrm{m}} 40^{\mathrm{s}} .53 /-71^{\circ} 58^{\prime} 03^{\prime \prime} 6$ & 7.7 & 1.9 & $1.1_{-0.3}^{+0.4} \times 10^{-13}$ & $\ldots$ & $\ldots$ & $\mathrm{U}$ & $\ldots$ \\
\hline SC700 & J005859.6-742410 & $00^{\mathrm{h}} 58^{\mathrm{m}} 59^{\mathrm{s}} \cdot 63 /-74^{\circ} 24^{\prime} 10^{\prime \prime} 5$ & 5.5 & 0.0 & $1.6_{-0.4}^{+0.4} \times 10^{-13}$ & $2.37_{-0.67}^{+1.07}$ & $\ldots$ & $\mathrm{U}$ & $\ldots$ \\
\hline SC702 & J011539.2-724055 & $01^{\mathrm{h}} 15^{\mathrm{m}} 39^{\mathrm{s}} \cdot 19 /-72^{\circ} 40^{\prime} 55^{\prime \prime} 8$ & 4.7 & 1.4 & $9.7_{-2.7}^{+3.1} \times 10^{-14}$ & $\ldots$ & $\ldots$ & $\mathrm{U}$ & $\ldots$ \\
\hline SC717 & J004614.4-740607 & $00^{\mathrm{h}} 46^{\mathrm{m}} 14^{\mathrm{s}} .37 /-74^{\circ} 06^{\prime} 07^{\prime \prime} 3$ & 4.6 & 4.4 & $4.2_{-1.6}^{+2.0} \times 10^{-14}$ & $\ldots$ & $\ldots$ & $\mathrm{U}$ & $\ldots$ \\
\hline SC720 & J012755.2-735713 & $01^{\mathrm{h}} 27^{\mathrm{m}} 55^{\mathrm{s}} \cdot 16 /-73^{\circ} 57^{\prime} 13^{\prime \prime} \cdot 7$ & 4.7 & 0.0 & $8.0_{-5.9}^{+8.1} \times 10^{-14}$ & $\cdots$ & $\ldots$ & $\mathrm{U}$ & $\ldots$ \\
\hline SC721 & J002818.4-733830 & $00^{\mathrm{h}} 28^{\mathrm{m}} 18^{\mathrm{s}} .37 /-73^{\circ} 38^{\prime} 30^{\prime \prime} 6$ & 5.2 & 2.4 & $1.1_{-0.3}^{+0.4} \times 10^{-13}$ & $1.91_{-0.99}^{+1.77}$ & $\ldots$ & $\mathrm{U}$ & $\ldots$ \\
\hline SC731 & J005754.7-715631 & $00^{\mathrm{h}} 57^{\mathrm{m}} 54^{\mathrm{s}} .73 /-71^{\circ} 56^{\prime} 31^{\prime \prime} 6$ & 6.1 & 2.1 & $1.3_{-0.3}^{+0.4} \times 10^{-13}$ & $\ldots$ & $\begin{array}{c}\text { 2MASS J00575428- } \\
7156306\end{array}$ & K & $\operatorname{AGN}(10,11)$ \\
\hline SC733 & J013307.5-740237 & $01^{\mathrm{h}} 33^{\mathrm{m}} 07^{\mathrm{s}} .52 /-74^{\circ} 02^{\prime} 37^{\prime \prime} \cdot 9$ & 5.3 & 6.1 & $3.3_{-1.0}^{+1.2} \times 10^{-13}$ & $1.60_{-1.20}^{+1.70}$ & $\ldots$ & $\mathrm{U}$ & $\ldots$ \\
\hline SC734 & J010036.2-714545 & $01^{\mathrm{h}} 00^{\mathrm{m}} 36^{\mathrm{s}} .19 /-71^{\circ} 45^{\prime} 45^{\prime \prime} .5$ & 5.1 & 5.0 & $7.0_{-2.6}^{+3.2} \times 10^{-14}$ & $\ldots$ & $\ldots$ & $\mathrm{U}$ & $\ldots$ \\
\hline SC738 & J004629.8-732608 & $00^{\mathrm{h}} 46^{\mathrm{m}} 29^{\mathrm{s}} .81 /-73^{\circ} 26^{\prime} 08^{\prime \prime} \cdot 7$ & 5.0 & 1.9 & $5.7_{-2.0}^{+2.4} \times 10^{-14}$ & $\ldots$ & $\ldots$ & $\mathrm{U}$ & $\ldots$ \\
\hline
\end{tabular}


Table 2

(Continued)

\begin{tabular}{|c|c|c|c|c|c|c|c|c|c|}
\hline SC\# & $\begin{array}{c}\text { Name } \\
\text { (1SCUBEDX J...) }\end{array}$ & $\begin{array}{l}\text { R.A./Decl. } \\
\text { (J2000) }\end{array}$ & $\begin{array}{l}\text { Err } \\
\left({ }^{\prime \prime}\right)\end{array}$ & $\begin{array}{l}\text { Det. } \\
(\%)\end{array}$ & $\begin{array}{r}f_{\text {mean }}(0.5-10 \mathrm{keV}) \\
\quad\left(\mathrm{erg} \mathrm{s}^{-1} \mathrm{~cm}^{-2}\right)\end{array}$ & $\Gamma$ & Catalog Name & $\mathrm{Cl}$. & $\begin{array}{c}\text { Type } \\
\text { (Reference) }\end{array}$ \\
\hline SC739 & J010156.6-724416 & $01^{\mathrm{h}} 01^{\mathrm{m}} 56^{\mathrm{s}} .57 /-72^{\circ} 44^{\prime} 16^{\prime \prime} 2$ & 4.6 & 6.0 & $8.4_{-2.8}^{+3.4} \times 10^{-14}$ & $\cdots$ & $\cdots$ & $\mathrm{U}$ & $\cdots$ \\
\hline SC740 & J011306.4-731504 & $01^{\mathrm{h}} 13^{\mathrm{m}} 06^{\mathrm{s}} .40 /-73^{\circ} 15^{\prime} 04^{\prime \prime} 3$ & 4.8 & 3.8 & $1.0_{-0.3}^{+0.3} \times 10^{-13}$ & $\ldots$ & $\ldots$ & $\mathrm{U}$ & $\ldots$ \\
\hline SC742 & J005447.3-721935 & $00^{\mathrm{h}} 54^{\mathrm{m}} 47^{\mathrm{s}} .34 /-72^{\circ} 19^{\prime} 35^{\prime \prime} 0$ & 4.9 & 2.1 & $1.6_{-0.7}^{+0.9} \times 10^{-13}$ & $\ldots$ & ... & $\mathrm{U}$ & $\ldots$ \\
\hline SC749 & J003750.0-723038 & $00^{\mathrm{h}} 37^{\mathrm{m}} 49^{\mathrm{s}} .99 /-72^{\circ} 30^{\prime} 38^{\prime \prime} \cdot 3$ & 5.6 & 5.0 & $2.4_{-1.2}^{+1.6} \times 10^{-13}$ & $\cdots$ & $\cdots$ & $\mathrm{U}$ & $\cdots$ \\
\hline SC755 & J004430.8-713418 & $00^{\mathrm{h}} 44^{\mathrm{m}} 30^{\mathrm{s}} \cdot 77 /-71^{\circ} 34^{\prime} 18^{\prime \prime} 8$ & 6.4 & 9.4 & $7.7_{-2.3}^{+2.8} \times 10^{-14}$ & $7.00_{-6.00}^{+67.00}$ & $\cdots$ & $\mathrm{U}$ & $\cdots$ \\
\hline SC761 & J005401.5-742727 & $00^{\mathrm{h}} 54^{\mathrm{m}} 01^{\mathrm{s}} .54 /-74^{\circ} 27^{\prime} 27^{\prime \prime} .4$ & 6.4 & 0.0 & $1.0_{-0.3}^{+0.3} \times 10^{-13}$ & $2.43_{-0.71}^{+2.43}$ & $\begin{array}{c}\text { MQS J005402.17- } \\
742733.3\end{array}$ & $\mathrm{~K}$ & $\operatorname{AGN}(8,10)$ \\
\hline SC764 & J010701.4-733449 & $01^{\mathrm{h}} 07^{\mathrm{m}} 01^{\mathrm{s}} .36 /-73^{\circ} 34^{\prime} 49^{\prime \prime} \cdot 2$ & 5.0 & 5.3 & $7.0_{-2.0}^{+2.4} \times 10^{-14}$ & $2.00_{-1.00}^{+1.40}$ & $\begin{array}{c}\text { MQS J010702.12- } \\
733445.3\end{array}$ & $\mathrm{U}$ & AGN (8) \\
\hline SC768 & J002804.9-730453 & $00^{\mathrm{h}} 28^{\mathrm{m}} 04^{\mathrm{s}} .86 /-73^{\circ} 04^{\prime} 53^{\prime \prime} 7$ & 7.8 & 5.6 & $7.5_{-2.8}^{+3.5} \times 10^{-13}$ & $\ldots$ & $\cdots$ & $\mathrm{U}$ & $\cdots$ \\
\hline SC769 & J003347.0-724229 & $00^{\mathrm{h}} 33^{\mathrm{m}} 46^{\mathrm{s}} .95 /-72^{\circ} 42^{\prime} 29^{\prime \prime} 0$ & 8.0 & 1.6 & $3.0_{-1.4}^{+1.8} \times 10^{-13}$ & $\ldots$ & $\cdots$ & $\mathrm{U}$ & $\ldots$ \\
\hline SC770 & J012814.6-725921 & $01^{\mathrm{h}} 28^{\mathrm{m}} 14^{\mathrm{s}} .56 /-72^{\circ} 59^{\prime} 21^{\prime \prime} 4$ & 4.6 & 3.3 & $5.9_{-1.9}^{+2.2} \times 10^{-14}$ & $\ldots$ & $\ldots$ & $\mathrm{U}$ & $\ldots$ \\
\hline SC776 & J011112.9-712036 & $01^{\mathrm{h}} 11^{\mathrm{m}} 12^{\mathrm{s}} \cdot 86 /-71^{\circ} 20^{\prime} 36^{\prime \prime} 5$ & 5.4 & 9.1 & $2.3_{-0.7}^{+0.8} \times 10^{-13}$ & $\ldots$ & ... & $\mathrm{U}$ & $\ldots$ \\
\hline SC788 & J005231.9-713238 & $00^{\mathrm{h}} 52^{\mathrm{m}} 31^{\mathrm{s}} .87 /-71^{\circ} 32^{\prime} 38^{\prime \prime} 8$ & 4.9 & 7.0 & $8.7_{-2.4}^{+2.9} \times 10^{-14}$ & $\cdots$ & ... & $\mathrm{U}$ & $\ldots$ \\
\hline SC790 & J004159.6-720513 & $00^{\mathrm{h}} 41^{\mathrm{m}} 59^{\mathrm{s}} .58 /-72^{\circ} 05^{\prime} 13^{\prime \prime} 1$ & 5.3 & 0.0 & $1.2_{-0.3}^{+0.4} \times 10^{-13}$ & $2.08_{-0.72}^{+2.12}$ & ... & $\mathrm{U}$ & $\ldots$ \\
\hline SC799 & J012639.6-733823 & $01^{\mathrm{h}} 26^{\mathrm{m}} 39^{\mathrm{s}} .57 /-73^{\circ} 38^{\prime} 23^{\prime \prime} 8$ & 5.3 & 2.3 & $9.1_{-2.9}^{+3.5} \times 10^{-14}$ & $1.40_{-1.10}^{+2.70}$ & $\ldots$ & $\mathrm{U}$ & $\ldots$ \\
\hline SC802 & J003553.8-725259 & $00^{\mathrm{h}} 35^{\mathrm{m}} 53^{\mathrm{s}} .80 /-72^{\circ} 52^{\prime} 59^{\prime \prime} 2$ & 5.9 & 2.6 & $1.2_{-0.3}^{+0.4} \times 10^{-13}$ & $\ldots$ & $\ldots$ & $\mathrm{U}$ & $\cdots$ \\
\hline SC806 & J012116.8-724414 & $01^{\mathrm{h}} 21^{\mathrm{m}} 16^{\mathrm{s}} .84 /-72^{\circ} 44^{\prime} 14^{\prime \prime} 4$ & 14.3 & 0.0 & $3.3_{-2.7}^{+4.3} \times 10^{-14}$ & $\ldots$ & $\begin{array}{c}\text { 3XMM J012116.6- } \\
724359\end{array}$ & K & $\ldots$ \\
\hline SC808 & J004810.4-723859 & $00^{\mathrm{h}} 48^{\mathrm{m}} 10^{\mathrm{s}} .40 /-72^{\circ} 38^{\prime} 59^{\prime \prime} 8$ & 4.7 & 2.4 & $4.5_{-1.7}^{+2.1} \times 10^{-14}$ & $\ldots$ & $\ldots$ & $\mathrm{U}$ & $\ldots$ \\
\hline SC813 & J011827.9-721741 & $01^{\mathrm{h}} 18^{\mathrm{m}} 27^{\mathrm{s}} .90 /-72^{\circ} 17^{\prime} 41^{\prime \prime} 8$ & 5.2 & 2.4 & $5.1_{-2.3}^{+2.9} \times 10^{-14}$ & $\ldots$ & $\ldots$ & $\mathrm{U}$ & $\ldots$ \\
\hline SC815 & J005911.1-713845 & $00^{\mathrm{h}} 59^{\mathrm{m}} 11^{\mathrm{s}} .06 /-71^{\circ} 38^{\prime} 45^{\prime \prime} 9$ & 5.5 & 3.7 & $2.8_{-0.9}^{+1.1} \times 10^{-13}$ & $0.27_{-0.95}^{+1.16}$ & SXP 2.76 & $\mathrm{~K}$ & $\operatorname{BeXRB}(1,2)$ \\
\hline SC817 & J013209.6-734033 & $01^{\mathrm{h}} 32^{\mathrm{m}} 09^{\mathrm{s}} \cdot 61 /-73^{\circ} 40^{\prime} 33^{\prime \prime} \cdot 1$ & 5.4 & 5.7 & $9.2_{-3.5}^{+4.4} \times 10^{-14}$ & $\ldots$ & ... & $\mathrm{U}$ & $\ldots$ \\
\hline
\end{tabular}

Note. Only sources that have been flagged as "Good" quality detections are listed in this table. References for source type: (1) Coe \& Kirk 2015; (2) Haberl \& Sturm 2016; (3) Azzopardi \& Vigneau 1979; (4) Meyssonnier \& Azzopardi 1993; (5) Massey 2002; (6) Kozłowski et al. 2011; (7) Kozłowski et al. 2012; (8) Kozłowski et al. 2013; (9) Véron-Cetty \& Véron 2010; (10) Cioni et al. 2013; (11) McGowan et al. 2008; (12) Sturm et al. 2013.

Table 3

Proposed Non-SXP HMXB Systems

\begin{tabular}{|c|c|c|c|c|c|c|c|}
\hline $\mathrm{SC} \#$ & HS\# & $\begin{array}{c}\text { Name } \\
(1 \text { SCUBEDX J...) }\end{array}$ & R.A./Decl. (J2000) & $\begin{array}{l}\text { Err } \\
\left({ }^{\prime \prime}\right)\end{array}$ & $\begin{array}{l}\text { Det. } \\
(\%)\end{array}$ & $\begin{array}{l}\text { Luminosity }^{\mathrm{a}} \\
(0.5-10 \mathrm{keV})\end{array}$ & Catalog Name \\
\hline$\overline{\mathrm{SC} 29}$ & 125 & J010155.5-723236 & $01^{\mathrm{h}} 01^{\mathrm{m}} 55^{\mathrm{s}} .53-72^{\circ} 32^{\prime} 36^{\prime \prime} 0$ & 4.9 & 7.3 & $3.4_{-1.4}^{+1.1} \times 10^{35}$ & AzV 285 \\
\hline SC161 & 121 & J010029.2-722033 & $01^{\mathrm{h}} 00^{\mathrm{m}} 29^{\mathrm{s}} \cdot 17-72^{\circ} 20^{\prime} 33^{\prime \prime} !^{1}$ & 5.3 & 11.4 & $1.9_{-0.8}^{+0.8} \times 10^{35}$ & [MA93] 1208 \\
\hline SC374 & 133 & J010435.6-722149 & $01^{\mathrm{h}} 04^{\mathrm{m}} 35^{\mathrm{s}} \cdot 57-72^{\circ} 21^{\prime} 49^{\prime \prime} \cdot 3$ & 8.2 & 10.0 & $4.9_{-2.2}^{+3.1} \times 10^{35}$ & [MA93] 1470 \\
\hline SC379 & 143 & J012326.7-732122 & $01^{\mathrm{h}} 23^{\mathrm{m}} 26^{\mathrm{s}} \cdot 67-73^{\circ} 21^{\prime} 22^{\prime \prime} 4$ & 6.7 & 7.0 & $2.3_{-1.0}^{+1.7} \times 10^{35}$ & [M2002] SMC 81035 \\
\hline SC404 & 76 & J004929.5-733107 & $00^{\mathrm{h}} 49^{\mathrm{m}} 29^{\mathrm{s}} \cdot 54-73^{\circ} 31^{\prime} 07 !{ }^{\prime \prime} 8$ & 6.7 & 4.5 & $4.4_{-1.1}^{+2.2} \times 10^{35}$ & [MA93] 302 \\
\hline $\mathrm{SC} 482$ & 128 & J010331.1-730141 & $01^{\mathrm{h}} 03^{\mathrm{m}} 31^{\mathrm{s}} .10-73^{\circ} 01^{\prime} 41^{\prime \prime} 2$ & 7.5 & 7.9 & $1.4_{-0.9}^{+0.6} \times 10^{35}$ & [M2002] SMC 56587 \\
\hline
\end{tabular}

Notes. The second column refers to matches with the catalog of Haberl \& Sturm (2016). The optical identifications come from the following catalogs: [MA93] (Meyssonnier \& Azzopardi 1993), AzV (Azzopardi \& Vigneau 1979), and [M2002] (Massey 2002).

${ }^{\text {a }}$ Luminosity is a mean value and assumes that the source is at a distance of $62 \mathrm{kpc}$.

Gotthelf et al. 1999). We created a model of the periodic emission from the S-CUBED light curve folded at the detected period, fitted by a DC level plus two Gaussians. Utilizing this model, we then generated simulated S-CUBED light curves, using the same observation times and exposures for each data point, assuming Poisson statistics. We finally performed a period search of all the simulated light curves. The quoted $1 \sigma$ error is the standard deviation of the measured peak period for 10,000 simulated light curves.

Table 4 lists all S-CUBED sources with detected periodicities, compared to reported orbital or superorbital periods found in the literature. Using the blind search $\left(P_{\text {false }} \leqslant 1 \%\right)$, we find five sources with significantly detected periods: SXP 91.1, SMC X-1, SXP 15.6, SXP 6.85, and SXP 46.6. In all cases the detected periods match within errors previously reported periodicities found from X-ray and optical data (citations given in Table 4), except for SXP 6.85 (see Section 6.2.4).

We note that $P_{\text {false }}$ is a measure that such a peak of a given height would occur in an $\mathrm{L}-\mathrm{S}$ periodogram in the case of a null hypothesis, and as such $P_{\text {false }}$ is not a valid measure of how likely a detection is to be real (VanderPlas 2018), especially in the case where an orbital period is already known to be present. 
Table 4

Detected Periodicities in S-CUBED Data, Compared with Known Periodicities in Sources

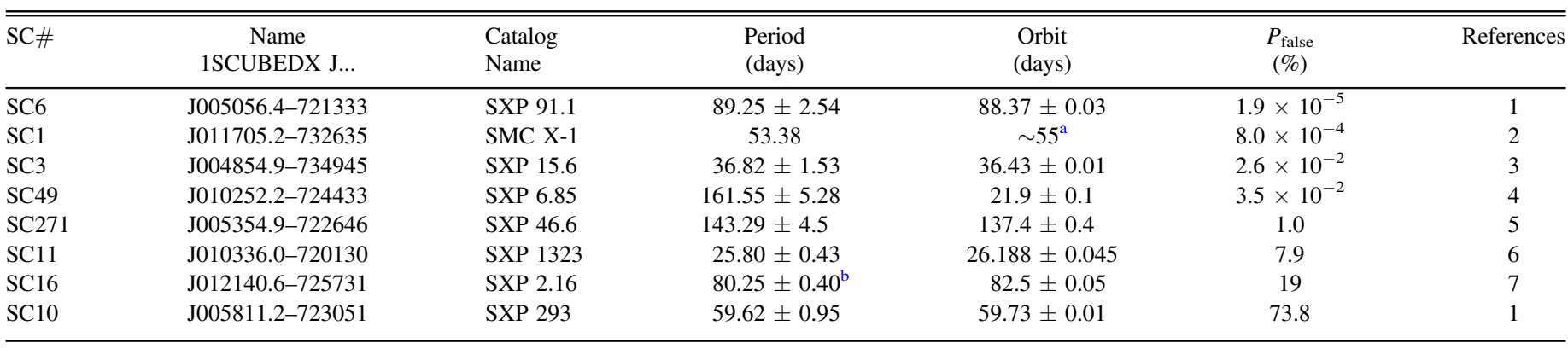

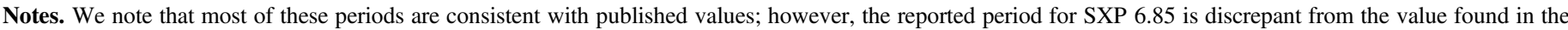

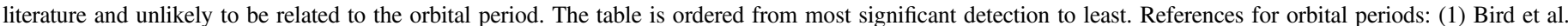

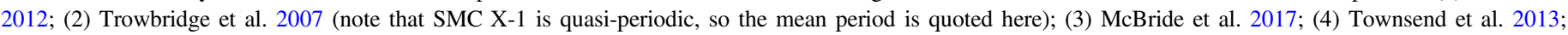
(5) Galache et al. 2008; (6) Carpano et al. 2017; (7) Boon et al. 2017 (reported period is from BAT data).

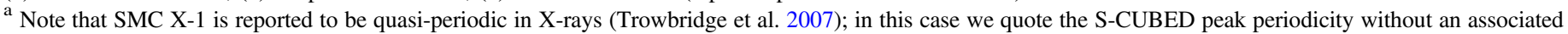
error, as the Monte Carlo simulation would not be valid for an object in which the period is not constant.

${ }^{\mathrm{b}}$ Error is likely underestimated for SXP 2.16, due to apparent turn-off of X-ray activity from this source after the final detection on 2016 October 5.

For this reason we also examined $\mathrm{L}-\mathrm{S}$ periodograms for all sources with a previously published period, looking for peaks in the periodogram near previously reported values. We report in Table 4 three sources for which the $\mathrm{L}-\mathrm{S}$ periodograms peak at periods close to their reported orbital periods, but with relatively low $P_{\text {false }}$ values: SXP 1323, SXP 2.16, and SXP 293. We believe that these period detections are likely real, despite their low $P_{\text {false }} \leqslant 1 \%$ values, given their consistency with published values. Ongoing observations of these objects with S-CUBED will likely increase the significance of their detection.

Given that these sources are BeXRBs, the likely source of the orbital modulation in these sources are periodic Type I outbursts, which occur during the periastron passages. An L-S search was able to detect the signature of these Type I outbursts, even though S-CUBED data are not of sufficient sensitivity or time resolution to resolve the shape of the individual outbursts themselves.

We note that sources with detected periods consistent with previously reported values lie in the range of 26.2-137.4 days. Therefore, nondetections from nine SXP sources can be explained by the orbital period being too long $(\geqslant 0.5 \mathrm{yr})$ or too short (3 weeks or less) to be detected. As an example, SXP 18.3, with a reported orbital period of 17.79 days (Coe et al. 2015), has a high DC (i.e., it is frequently detected), but no obvious periodic variability has been detected.

Several SXP sources have frequent detections and previously reported orbital periods in the expected highest sensitivity range of 21-180 days, but no period detection in S-CUBED. For example, SXP 327, SXP 967, SXP 169, SXP 175, and SXP 264 all have orbital periods in the right range, but no detected period.

The lack of a period detection in SXP 175 and SXP 172 is especially surprising, given that their orbital periods, 87.2 and 68.8 days, respectively, lie in the middle of the highest sensitivity period range and have DCs of $22 \%$ and $32 \%$, which places them between other sources that have period detections in Figure 7.

In the case of SXP 172, the orbital period has been previously been measured in X-ray by RXTE in data taken frequently between 1999 and 2009 (Schurch et al. 2011). In
RXTE data these outbursts peak between $\sim 0.1$ and -0.5 PCA counts $\mathrm{PCU}^{-1} \mathrm{~s}^{-1}$, which, assuming a type BeXRB spectrum, converts to a count rate of $\sim 0.04$ XRT count $\mathrm{s}^{-1}$, or around 2.3 counts in a $60 \mathrm{~s}$ exposure. Therefore, it is possible that Type I bursts are too faint to detect in S-CUBED data.

\section{Sources of Interest}

In this section we report on sources of particular interest from the first year of monitoring. We define interesting sources as those that are detected in $>20 \%$ of all observations and those that are considered highly variable. Variability in this case is Pearson's $\chi^{2}$, i.e., we fit a model of a constant level to each S-CUBED light curve (points are weighted by their measurement errors). We then find a reduced $\chi^{2}\left(\chi_{\text {red }}^{2}\right)$ for the constant level fit, and $\chi_{\text {red }}^{2}>2$ is considered to be highly variable. These sources of interest are given in Table 5. In this table, we also present a mean luminosity level for each source, as estimated by performing a simple power-law fit to the spectrum of the combined S-CUBED observations, correcting for absorption, and assuming a standard SMC distance of $62 \mathrm{kpc}$. The majority of the "Sources of Interest" based on these criteria are previously identified BeXRBs.

\subsection{Uncataloged Sources}

Two sources have been found to be frequently ( $>20 \%$ of the time) detected by S-CUBED that are previously uncataloged X-ray emitters: 1SCUBEDX J003235.5-730650 (SC8) and 1SCUBEDX J003108.2-731207 (SC143). In this section we take a closer look at these two objects, in order to determine their source type.

\subsubsection{SCUBEDX J003235.5-730650}

S-CUBED detected 1SCUBEDX J003235.5-730650 (SC8) in $71.8 \%$ of all observations performed in the first year of observations. The Swift/XRT-derived position does not match any known cataloged X-ray point source, or any known optical sources. In order to investigate the nature of this object, ToO observations were performed by Swift for a total exposure of 12.3 ks between 2016 July 7 and August 29, as well as by 
Table 5

Sources of Interest in the S-CUBED Catalog, Ordered by Date of First Detection

\begin{tabular}{|c|c|c|c|c|c|c|c|c|c|}
\hline $\mathrm{SC} \#$ & $\begin{array}{c}\text { Name } \\
\text { (1SCUBEDX J...) }\end{array}$ & First Detection & R.A./Decl. (J2000) & $\begin{array}{l}\text { Err } \\
(")\end{array}$ & $\begin{array}{l}\text { Det. } \\
(\%)\end{array}$ & $\begin{array}{l}\text { Luminosity }^{\mathrm{a}} \\
(0.5-10 \mathrm{keV})\end{array}$ & Catalog Name & Type & References \\
\hline$\overline{\mathrm{SC} 1}$ & J011705.2-732635 & 2016 Jun 08 & $01^{\mathrm{h}} 17^{\mathrm{m}} 05^{\mathrm{s}} \cdot 18 /-73^{\circ} 26^{\prime} 35^{\prime \prime} 8$ & $2.2^{\mathrm{b}}$ & 100.0 & $1.7_{-0.1}^{+0.1} \times 10^{38}$ & SMC X-1 & HMXB & (1) \\
\hline $\mathrm{SC} 2$ & J010401.3-720155 & 2016 Jun 09 & $01^{\mathrm{h}} 04^{\mathrm{m}} 01^{\mathrm{s}} \cdot 29 /-72^{\circ} 01^{\prime} 55^{\prime \prime} \cdot 7$ & 3.8 & 100.0 & $\cdots$ & $\begin{array}{l}\text { 1E } 0102.2 \\
-7219\end{array}$ & SNR & \\
\hline $\mathrm{SC} 3$ & J004854.9-734945 & 2016 Jun 24 & $00^{\mathrm{h}} 48^{\mathrm{m}} 54^{\mathrm{s}} \cdot 93 /-73^{\circ} 49^{\prime} 45^{\prime \prime} 6$ & 3.8 & 59.1 & $1.9_{-0.3}^{+0.4} \times 10^{36}$ & SXP 15.6 & BeXRB & $(1,2)$ \\
\hline SC5 & J004910.3-724939 & 2016 Jun 24 & $00^{\mathrm{h}} 49^{\mathrm{m}} 10^{\mathrm{s}} \cdot 29 /-72^{\circ} 49^{\prime} 39^{\prime \prime} 2$ & 4.1 & 78.0 & $3.8_{-0.5}^{+0.6} \times 10^{36}$ & SXP 18.3 & BeXRB & \\
\hline SC6 & J005056.4-721333 & 2016 Jun 24 & $00^{\mathrm{h}} 50^{\mathrm{m}} 56^{\mathrm{s}} \cdot 39 /-72^{\circ} 13^{\prime} 33^{\prime \prime} 4$ & 4.6 & 43.2 & $1.3_{-0.3}^{+0.4} \times 10^{36}$ & SXP 91.1 & BeXRB & \\
\hline SC8 & J003235.5-730650 & 2016 Jun 28 & $00^{\mathrm{h}} 32^{\mathrm{m}} 35^{\mathrm{s}} \cdot 46 /-73^{\circ} 06^{\prime} 50^{\prime \prime} 9$ & $2.3^{\mathrm{b}}$ & 71.4 & $1.5_{-0.2}^{+0.1} \times 10^{-12}$ & & AGN & (3) \\
\hline SC10 & J005811.2-723051 & 2016 Jun 28 & $00^{\mathrm{h}} 58^{\mathrm{m}} 11^{\mathrm{s}} \cdot 15 /-72^{\circ} 30^{\prime} 51^{\prime \prime} 0$ & 4.6 & 32.0 & $1.1_{-0.3}^{+0.3} \times 10^{36}$ & SXP 293 & BeXRB & \\
\hline $\mathrm{SC} 11$ & J010336.0-720130 & $2016 \mathrm{Jul} 04$ & $01^{\mathrm{h}} 03^{\mathrm{m}} 36^{\mathrm{s}} \cdot 00 /-72^{\circ} 01^{\prime} 30^{\prime \prime} 8$ & 5.4 & 36.4 & $7.8_{-2.3}^{+2.8} \times 10^{35}$ & SXP 1323 & BeXRB & \\
\hline $\mathrm{SC} 13$ & J005455.4-724513 & $2016 \mathrm{Jul} 04$ & $00^{\mathrm{h}} 54^{\mathrm{m}} 55^{\mathrm{s}} \cdot 42 /-72^{\circ} 45^{\prime} 13 !^{\prime \prime} 1$ & 4.0 & 39.1 & $6.3_{-1.0}^{+1.2} \times 10^{35}$ & SXP 504 & BeXRB & \\
\hline SC16 & J012140.6-725731 & $2016 \mathrm{Jul} 06$ & $01^{\mathrm{h}} 21^{\mathrm{m}} 40^{\mathrm{s}} \cdot 63 /-72^{\circ} 57^{\prime} 31^{\prime \prime} 9$ & $3.2^{\mathrm{b}}$ & 13.6 & $7.4_{-1.8}^{+3.7} \times 10^{35}$ & SXP 2.16 & BeXRB & (4) \\
\hline $\mathrm{SC} 17$ & J010428.3-723135 & $2016 \mathrm{Jul} 06$ & $01^{\mathrm{h}} 04^{\mathrm{m}} 28^{\mathrm{s}} \cdot 32 /-72^{\circ} 31^{\prime} 35^{\prime \prime} 3$ & 5.1 & 28.3 & $1.0_{-0.2}^{+0.2} \times 10^{36}$ & SXP 707 & BeXRB & \\
\hline $\mathrm{SC} 20$ & J005919.8-722317 & $2016 \mathrm{Jul} 06$ & $00^{\mathrm{h}} 59^{\mathrm{m}} 19^{\mathrm{s}} \cdot 82 /-72^{\circ} 23^{\prime} 17^{\prime \prime} 9$ & 4.3 & 40.0 & $1.4_{-0.2}^{+0.3} \times 10^{36}$ & SXP 202A & BeXRB & (5) \\
\hline $\mathrm{SC} 32$ & J011838.2-732533 & 2016 Aug 02 & $01^{\mathrm{h}} 18^{\mathrm{m}} 38^{\mathrm{s}} \cdot 21 /-73^{\circ} 25^{\prime} 33 !^{\prime \prime} 7$ & $7.1^{\mathrm{b}}$ & 47.5 & $6.0_{-2.3}^{+20.8} \times 10^{-12}$ & HD $8191 \mathrm{~A} / \mathrm{B}$ & FG-star & \\
\hline SC49 & J010252.2-724433 & 2016 Aug 02 & $01^{\mathrm{h}} 02^{\mathrm{m}} 52^{\mathrm{s}} \cdot 17 /-72^{\circ} 44^{\prime} 333^{\prime \prime} 4$ & 4.4 & 55.2 & $2.3_{-0.3}^{+0.4} \times 10^{36}$ & SXP 6.85 & BeXRB & (1) \\
\hline SC71 & J005205.3-722603 & 2016 Aug 02 & $00^{\mathrm{h}} 52^{\mathrm{m}} 05^{\mathrm{s}} \cdot 27 /-72^{\circ} 26^{\prime} 03^{\prime \prime} 8$ & 4.3 & 71.4 & $1.9_{-0.1}^{+0.2} \times 10^{37}$ & SMC X-3 & BeXRB & $(6,7,8)$ \\
\hline SC 80 & J005052.5-710902 & 2016 Aug 02 & $00^{\mathrm{h}} 50^{\mathrm{m}} 52^{\mathrm{s}} \cdot 47 /-71^{\circ} 09^{\prime} 02^{\prime \prime} 3$ & 6.8 & 59.5 & - & HD 5028 & FG-star & \\
\hline SC143 & J003108.2-731207 & 2016 Aug 04 & $00^{\mathrm{h}} 31^{\mathrm{m}} 08^{\mathrm{s}} \cdot 18 /-73^{\circ} 12^{\prime} 07^{\prime \prime} 3$ & 4.1 & 20.8 & $1.1_{-0.6}^{+2.0} \times 10^{36}$ & & Unknown & \\
\hline SC148 & J005151.6-731031 & 2016 Aug 08 & $00^{\mathrm{h}} 51^{\mathrm{m}} 51^{\mathrm{s}} \cdot 56 /-73^{\circ} 10^{\prime} 311^{\prime \prime} 5$ & 7.4 & 30.8 & $4.9_{-1.1}^{+4.5} \times 10^{35}$ & SXP 172 & BeXRB & \\
\hline SC160 & J010151.2-722334 & 2016 Aug 09 & $01^{\mathrm{h}} 01^{\mathrm{m}} 51^{\mathrm{s}} \cdot 19 /-72^{\circ} 23^{\prime} 34^{\prime \prime} 6$ & 5.0 & 21.7 & $6.0_{-1.3}^{+1.5} \times 10^{35}$ & SXP 175 & BeXRB & \\
\hline $\mathrm{SC} 271$ & J005354.9-722646 & 2016 Sep 22 & $00^{\mathrm{h}} 53^{\mathrm{m}} 54^{\mathrm{s}} \cdot 94 /-72^{\circ} 26^{\prime} 46^{\prime \prime} 1$ & 4.4 & 23.5 & $1.2_{-0.2}^{+0.2} \times 10^{36}$ & SXP 46.6 & BeXRB & \\
\hline SC372 & J005212.9-731916 & 2016 Nov 24 & $00^{\mathrm{h}} 52^{\mathrm{m}} 12^{\mathrm{s}} \cdot 88 /-73^{\circ} 19^{\prime} 16^{\prime \prime} 7$ & 4.8 & 17.2 & $8.8_{-0.6}^{+0.5} \times 10^{36}$ & SXP 15.3 & BeXRB & \\
\hline SC403 & J005456.4-722647 & 2016 Dec 09 & $00^{\mathrm{h}} 54^{\mathrm{m}} 56^{\mathrm{s}} .43 /-72^{\circ} 26^{\prime} 47^{\prime \prime} 7$ & 4.0 & 20.0 & $3.1_{-0.4}^{+0.4} \times 10^{36}$ & SXP 59.0 & BeXRB & (9) \\
\hline
\end{tabular}

Notes. In this case interesting sources are defined as those that are frequently ( $>20 \%$ of observations) detected or highly variable $\left(\chi_{\text {red }}^{2}>2\right.$ and detected in $>10 \%$ of observations).

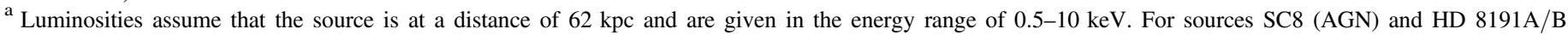

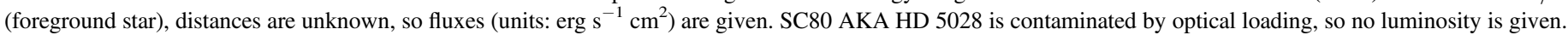
1E 0102.2-7219 (SC2) is an extended source, so the S-CUBED calculated luminosity is not correct and therefore is omitted.

b Position is enhanced utilizing UVOT to correct for systematic errors in astrometery using the method of Goad et al. (2007).

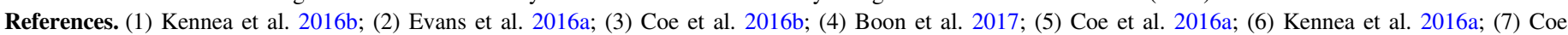
et al. 2016c; (8) Townsend et al. 2017; (9) Kennea et al. 2017.

NuSTAR (Observation ID 90201030002) on 2016 July 17 for $\sim 55 \mathrm{ks}$ and Chandra (Observation ID 19691) on 2016 August 11 for $\sim 1 \mathrm{ks}$. Analysis of these observations has been previously reported by Coe et al. (2016b).

The NuSTAR observation was performed primarily to investigate the possibility that this source was either an accreting pulsar or a magnetar, by searching for any pulsar periodicity. However, analysis of the NUSTAR data does not reveal the presence of any significant temporal variations. Spectral analysis of the NUSTAR data reveals that the source is well fit using a power-law model (XSPEC tbabs * power model), with a photon index of $1.65_{-0.10}^{+0.15}$ with a NuSTAR flux of $2 \times 10^{-12} \mathrm{erg} \mathrm{s}^{-1} \mathrm{~cm}^{-2}$ (3-78 keV); given NuSTAR's $3 \mathrm{keV}$ low energy cutoff, the absorption is not well constrained for these data. The averaged spectrum from combined S-CUBED data was well fit by a photon index of $1.53_{-0.09}^{+0.21}$, consistent within errors with the NuSTAR spectrum; the absorption is consistent with the Galactic value of $1.93 \times 10^{21} \mathrm{~cm}^{-2}$ (Willingale et al. 2013).

The lack of an optical counterpart consistent with the XRTderived position was an enigma. Analysis of the ToO XRT data revealed a large disparity (13!.5) between the XRT position derived using XRT-only data and the position utilizing UVOT data to correct for astrometric errors (Goad et al. 2007). This disparity motivated a short $\sim 1$ ks observation with Chandra, in order to obtain a better localization of the source. The Chandra position for 1SCUBEDX J003235.5-730650 was found to be R.A./decl.(J2000) $=00^{\mathrm{h}} 32^{\mathrm{m}} 34^{\mathrm{s}} .72-73^{\circ} 06^{\prime} 49^{\prime \prime}$. 14 . This position lies 3!"7 from the best S-CUBED-derived position given in Table 5, outside of the 2 " 3 radius $90 \%$ confidence error radius. We note that the Chandra position is consistent with the XRTonly-derived position from the ToO data. This new position is consistent with the location of an optical source seen in OGLE III and OGLE IV data, with an apparent nonstellar point-spread function (Coe et al. 2016b), suggesting that this source is likely a background AGN. We also note that this object is in the AllWISE AGN catalog (Secrest et al. 2015) and that the powerlaw spectrum detected by Swift and NuSTAR is consistent with the AGN hypothesis.

\subsubsection{SCUBEDX J003108.2-731207}

1SCUBEDX J003108.2-731207 (SC143) was detected in $20.8 \%$ of S-CUBED observations and has a mean flux of $2.3_{-1.5}^{+4.3} \times 10^{-13} \mathrm{erg} \mathrm{s}^{-1} \mathrm{~cm}^{-2}(0.5-10 \mathrm{keV}$, correct for absorption). The average spectrum combining all S-CUBED data reveals a soft spectrum, with a power-law fit giving a photon index of $4.8_{-1.2}^{+1.8}$, suggesting that the source is not a BeXRB, which typically have hard $(\Gamma \simeq 1.0)$ spectra. However, the power-law fit 
also requires a high absorption $\left(0.49_{0.22}^{+0.33} \times 10^{22} \mathrm{~cm}^{-2}\right)$, which, given the low line-of-sight absorption in the SMC, would require the absorption to be localized to the source. As an alternative, fitting a thermal (XSPEC's apec) model provides a good fit to the data with $N_{\mathrm{H}}$ fixed at the expected SMC value, with $\chi_{\text {red }}^{2}=0.924$ (13 dof), which is an improved fit over the absorbed power-law model $\left(\chi_{\text {red }}^{2}=1.126\right.$ for 13 dof $)$. This gives a fitted $k T=0.74_{-0.18}^{+0.19} \mathrm{keV}$, with a metallic abundance of $0.13_{-0.09}^{+0.23}$.

The fitted average flux of 1SCUBEDX J003108.2-731207 is $1.06_{-1.01}^{+0.41} \times 10^{-13} \mathrm{erg} \mathrm{s}^{-1} \mathrm{~cm}^{-2}(0.5-10 \mathrm{keV})$, equivalent to $\sim 4.5 \times 10^{34} \mathrm{erg} \mathrm{s}^{-1}$ at $62 \mathrm{kpc}$.

A catalog search reveals no known X-ray point sources at or near the S-CUBED position. However, we note that this source is inside the same pointing as source SC8, for which the S-CUBEDderived position was not consistent with a Chandra position. Unfortunately, SC143 is outside of the FOV of the Chandra observation; however, an XRT-only position derived from ToO observations was found to be consistent with the Chandra localization, so we utilized these data to calculate an updated position for SC143, which was found to be R.A./decl.(J2000) = $00^{\mathrm{h}} 31^{\mathrm{m}} 08.59-73^{\circ} 12^{\prime} 06^{\prime \prime} .4$ with an estimated error of $4^{\prime \prime}(90 \%$ confidence).

A catalog search reveals several possible optical and IR counterparts at this position. A nearby bright $(J=11.651)$ source 2MASS J00310958-7312082 (Cutri et al. 2003) lies 4.7" from the best XRT position, just outside the error circle, and is likely a good candidate to be the optical counterpart of SC143. However, Kato et al. (2007) list two fainter $(J=19.08$ and $J=18.88$ ) point sources inside the XRT error circle that could also be associated with the source. Given the uncertainty with the astrometry in this field, it is clear that we cannot definitively associate an optical/IR counterpart with this source. Therefore, further study of this source with deeper X-ray observations and a positive identification of the optical/ IR counterpart will be required in order to classify the source type for SC143.

\subsection{Sources Showing Significant Outbursts or Variability}

Figure 10 shows light curves of the sources in Table 5 that show a statistically significant (reduced $\chi^{2}>2.0$ when fit with a constant model) degree of variability. In the following subsections, we discuss the details of individual outbursting sources, including analysis of ToO observations from Swift and other observatories, if those results have not been previously published.

\subsubsection{SMC $X-1$}

SMC X-1 is an HMXB containing an X-ray pulsar with a 0.71 s period; the companion star is a B0 supergiant (e.g., Li \& van den Heuvel 1997). SMC X-1 is a persistent X-ray emitter but shows large quasi-periodic superorbital variations with an average period of $\sim 55$ days (Trowbridge et al. 2007), which can be clearly seen in Figure 10. SMC X-1 is the only variable source in the S-CUBED survey to be detected in all observations. The S-CUBED detection of SMC X-1 was previously reported by Kennea et al. (2016b).

The BAT Transient Monitor (Krimm et al. 2013) detects SMC X-1 daily. In Figure 11, we compare the count rate seen in those data with the S-CUBED-derived count rate. It is clear that the S-CUBED data, although much more poorly sampled than the BAT Transient Monitor data, which are plotted here with 1-day time resolution, closely follow the superorbital variations of SMC X-1. L-S analysis of the BAT Transient Monitor data reveals a peak in the periodogram at 53.5 days, and analysis of the sparser S-CUBED observation data shows a peak in the periodogram at 53.4 days, i.e., the periods measured by BAT and S-CUBED are consistent, as expected.

\subsubsection{SMC $X-3$}

SMC X-3 is a BeXRB source in the SMC, first discovered by the $S A S 3$ satellite in 1977 (Li et al. 1977; Clark et al. 1978). In 2002, RXTE detected a bright outbursting pulsar in the SMC with a $\sim 7.8 \mathrm{~s}$ periodicity, although due to the localization accuracy of $R X T E$, it was not possible to associate this with SMC X-3. Edge et al. (2004), utilizing Chandra observations, accurately localized the $R X T E$-discovered pulsar and confirmed that it was indeed a new outburst of SMC X-3.

SMC X-3 is known to have large Type II outbursts; however, no outburst from the source had been seen during the Swift mission lifetime, although the source had been observed in quiescence several times by Swift. SMC X-3 was detected during the first S-CUBED observation on 2016 June 8. S-CUBED observed SMC X-3 five times between 2016 June 24 and July 16 (see Table 1), but it was not detected in any of those observations.

SMC X-3 was detected again on 2016 July 30, 5 times brighter than the previous 2016 June 8 detection. The brightening continued, showing that the source had entered into a significant likely Type II outburst. Unfortunately at this time, due to an error in the analysis software, this brightening was not flagged and therefore went unnoticed. However, 2 days previously, on 2016 August 8, MAXI reported the detection of a bright new transient, named MAXI J0058-721, in the SMC consistent with the location of SMC X-3 (Negoro et al. 2016), but also with SXP 6.85 (SC49 in Table 5), which was undergoing an outburst at the time (Kennea et al. 2016b).

The S-CUBED observations of 2016 August 8 confirmed that MAXI J0057-721 was in fact SMC X-3 entering a new outburst (Kennea et al. 2016a). S-CUBED monitoring (see Figure 10) tracked the outburst of SMC X-3. In addition to S-CUBED monitoring, additional Swift ToO observations were performed in WT mode in order to track the evolution of the pulsar periodicity, which not only confirmed the presence of the $\sim 7.8 \mathrm{~s}$ period but also allowed the accretion-powered spin-up of the pulsar to be measured. Results of those observations are reported by Townsend et al. (2017) and additionally by Tsygankov et al. (2017), Weng et al. (2017), and Koliopanos \& Vasilopoulos (2018). By measuring the effects of orbit-induced Doppler shift on the spin period of SMC X-3, these data provided for the first time a dynamical measure of the orbital period, $P_{\text {orb }}=45.04 \pm$ 0.08 days, and eccentricity, $e=0.244 \pm 0.005$ (values quoted from Townsend et al. 2017).

Although the outburst of SMC X-3 appears to have ended in late 2017 February (S-CUBED-measured flux level returned to 0.1 counts $\mathrm{s}^{-1}$, consistent with its pre-outburst level on the observation of 2017 February 22), it continued to be detected, although the detections appear interspersed with periods of nondetection. Examining combined S-CUBED and ToO observations performed by Swift in PC mode, which were requested as part of the additional monitoring of SMC X-3, reveals that these 

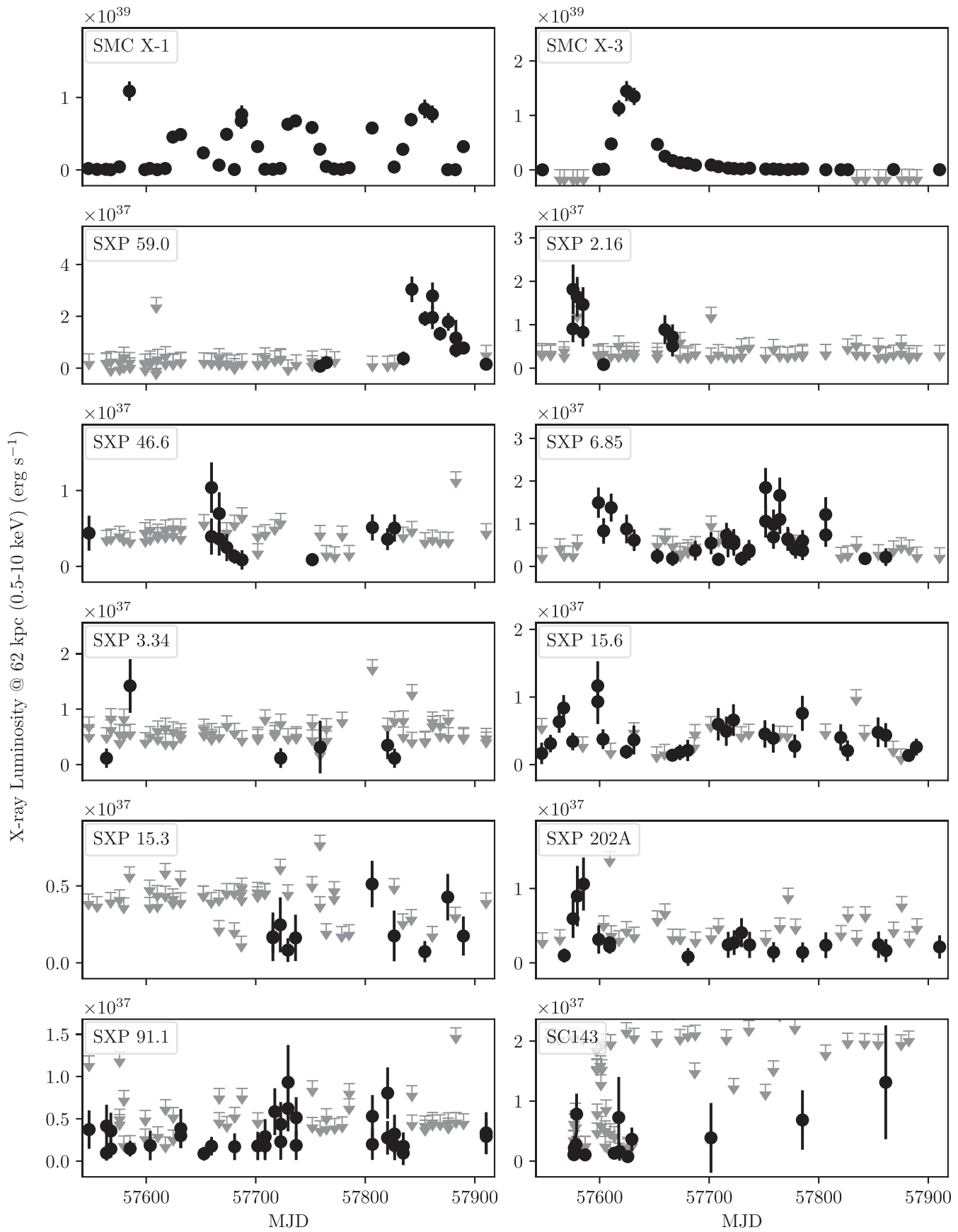

Figure 10. S-CUBED light curves for flaring and variable sources in the SMC during the first year of S-CUBED. Upper limits for nondetections are shown in light gray.

detections are in fact low-level periodic outbursts, peaking every $\sim 45$ days, i.e., the orbital period of SMC X-3.

Figure 12 shows the post-outburst light curve of SMC X-3, with the predicted periastron passages using the orbital ephemeris reported by Townsend et al. (2017) shown. These periodic flares therefore represent the signature of BeXRB Type I outbursts from SMC X-3, peaking at the orbital periastron. These Type I bursts have been reported at a similar level in pre-outburst Swift data by Tsygankov et al. (2017) and therefore show no evidence of enhancement related to the large Type II outburst. Figure 12 shows the post-outburst Type I bursts, which peak between $\sim 4 \times 10^{36}$ and $\sim 10^{37} \mathrm{erg} \mathrm{s}^{-1}(0.5-10 \mathrm{keV})$, consistent with the typical range of outburst luminosities seen in Type I outbursts from BeXRBs (e.g., Stella et al. 1986).
6.2.3. SXP 59.0

SXP 59.0 (1SCUBEDX J005456.4-722647, S-CUBED source SC403) is a BeXRB system, first identified as an $\mathrm{X}$-ray pulsar in outburst by $R X T E$ in observations taken on 1998 January 20, with a measured period of $59.0 \pm 0.2 \mathrm{~s}$ and designated XTE J0055-724 (Marshall et al. 1998). SXP 59.0 has a reported orbital period of $122.1 \pm 0.38$ days (Galache et al. 2008) based on RXTE observations, and an independently derived optical period of 122.25 days, consistent with the $R X T E$ period, was reported from analysis of OGLE I-band light curves by Bird et al. (2012).

S-CUBED detected an outburst of SXP 59.0 starting on 2017 March 30 (Kennea et al. 2017). As a result of this outburst, Swift ToO observations were requested to perform 


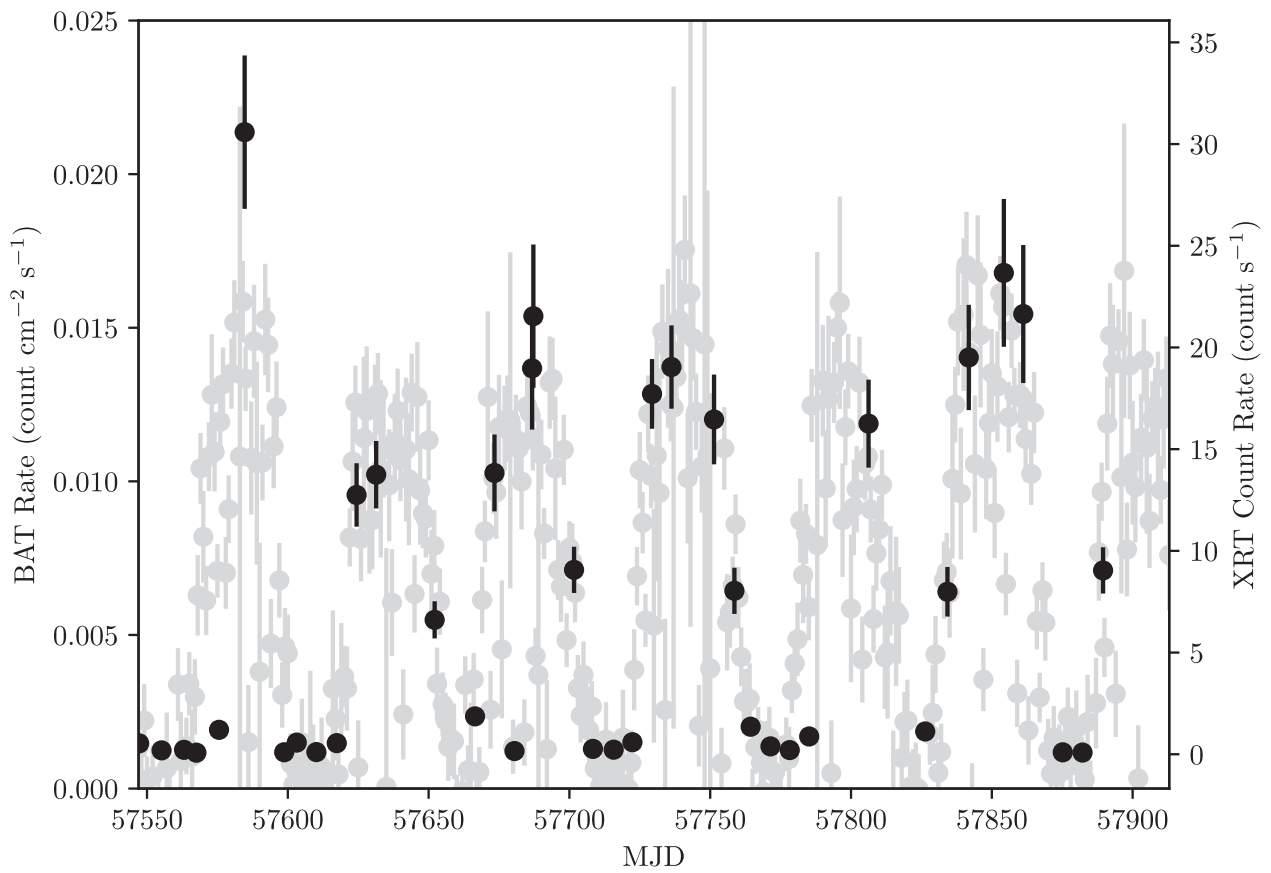

Figure 11. Comparison of the BAT Transient Monitor light curve of SMC X-1 (light gray) and the S-CUBED detections (black).

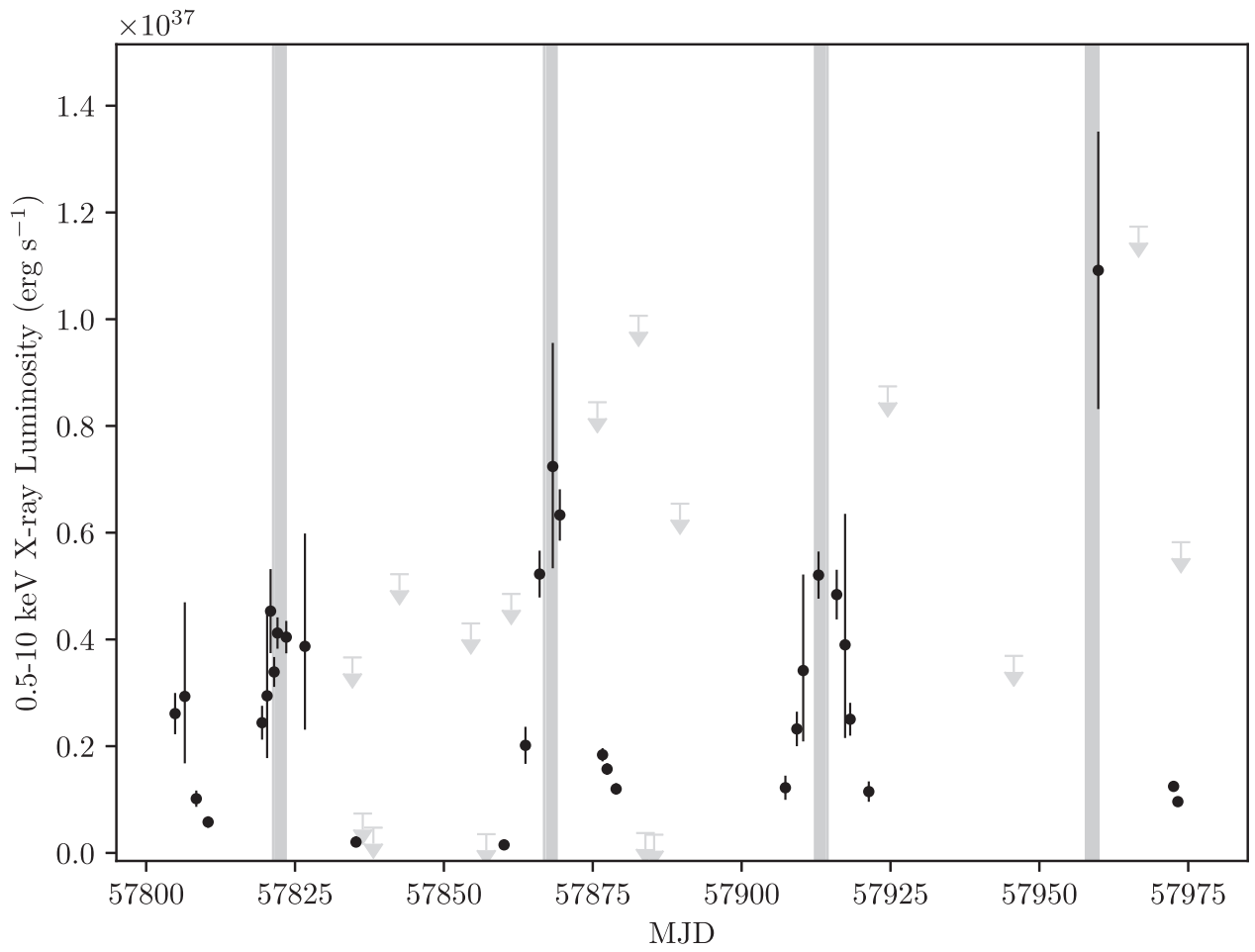

Figure 12. Post-outburst monitoring of SMC X-3 by S-CUBED, with additional Swift/XRT data taken as part of a ToO campaign. Repeated flares at the orbital period of $\sim 45$ days are the signature of Type I outbursts from this BeXRB source. Predicted periastron passages, derived from the orbital ephemeris of Townsend et al. (2017), are shown as vertical lines.

higher-cadence and more sensitive monitoring of the outburst. WT mode was requested in order to avoid pileup and also to help with detection of the pulsar period. WT mode observations were taken between 2017 April 12 and 30, every 3 days, with a requested observation time of $3 \mathrm{ks}$ per observation, although the actual exposure times varied owing to scheduling issues. In addition to these WT observations, several deep exposures in PC mode were taken during this period in coordination with
NUSTAR observations of the SMC and SXP 59.0. S-CUBED observations continued to be taken during this period also. The combined light curve of S-CUBED and ToO (including serendipitous) observations is shown in Figure 13.

The outburst was observed to peak during a ToO observation on 2017 April 07 at $L_{\mathrm{X}}=4.573_{-0.168}^{+0.159} \times 10^{37} \mathrm{erg} \mathrm{s}^{-1}$, approximately $26 \% L_{\text {Edd }}$ for a $1.4 M_{\odot}$ NS. After peak, the outburst declines approximately exponentially with a time constant of 


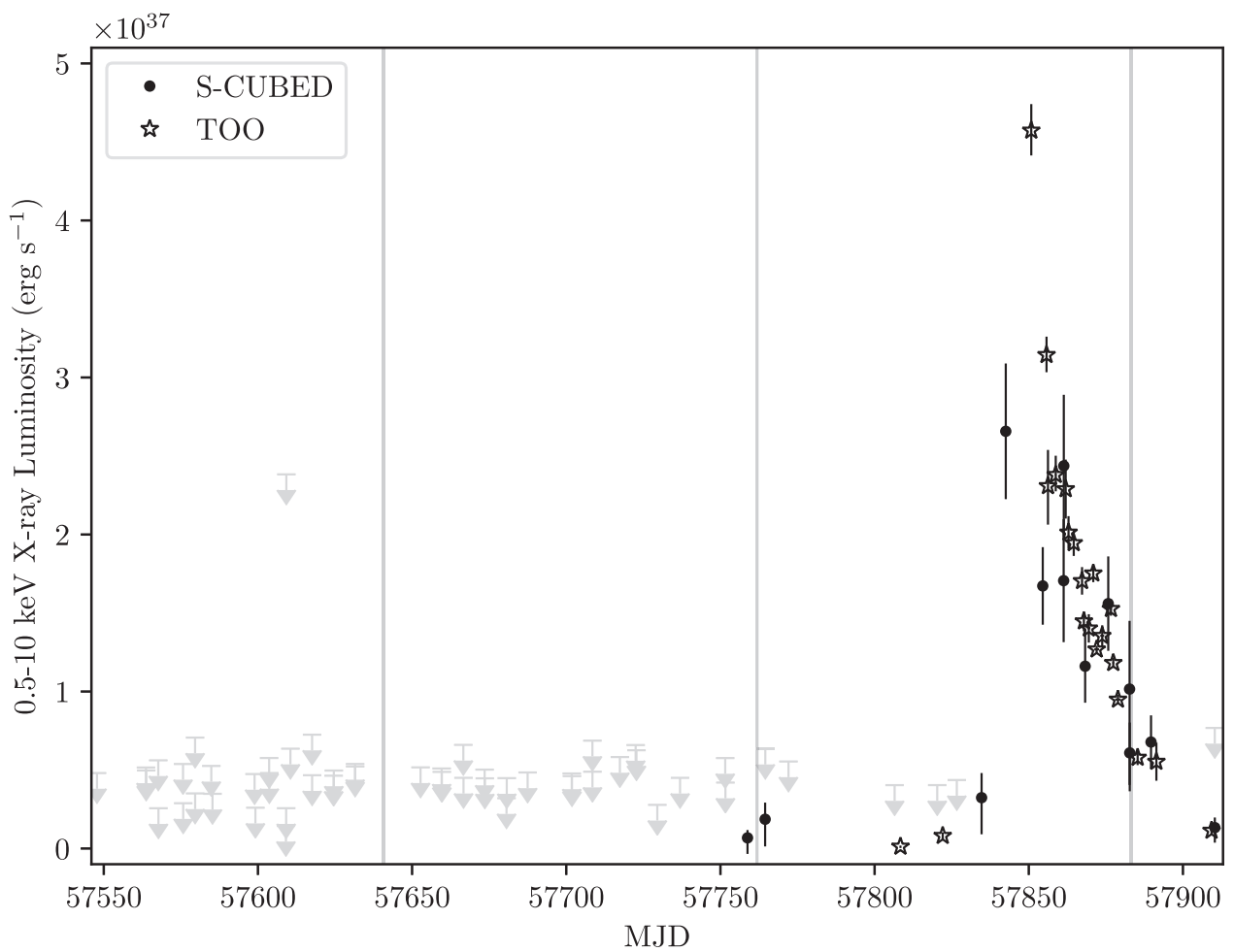

Figure 13. Light curve of combined S-CUBED (circles) and Swift ToO observations (stars) of SXP 59.0, which showed a bright Type II outburst starting around 2017 March 30. The source appears to have returned to the pre-outburst observation level at the time of the final S-CUBED observation on 2017 June 6.

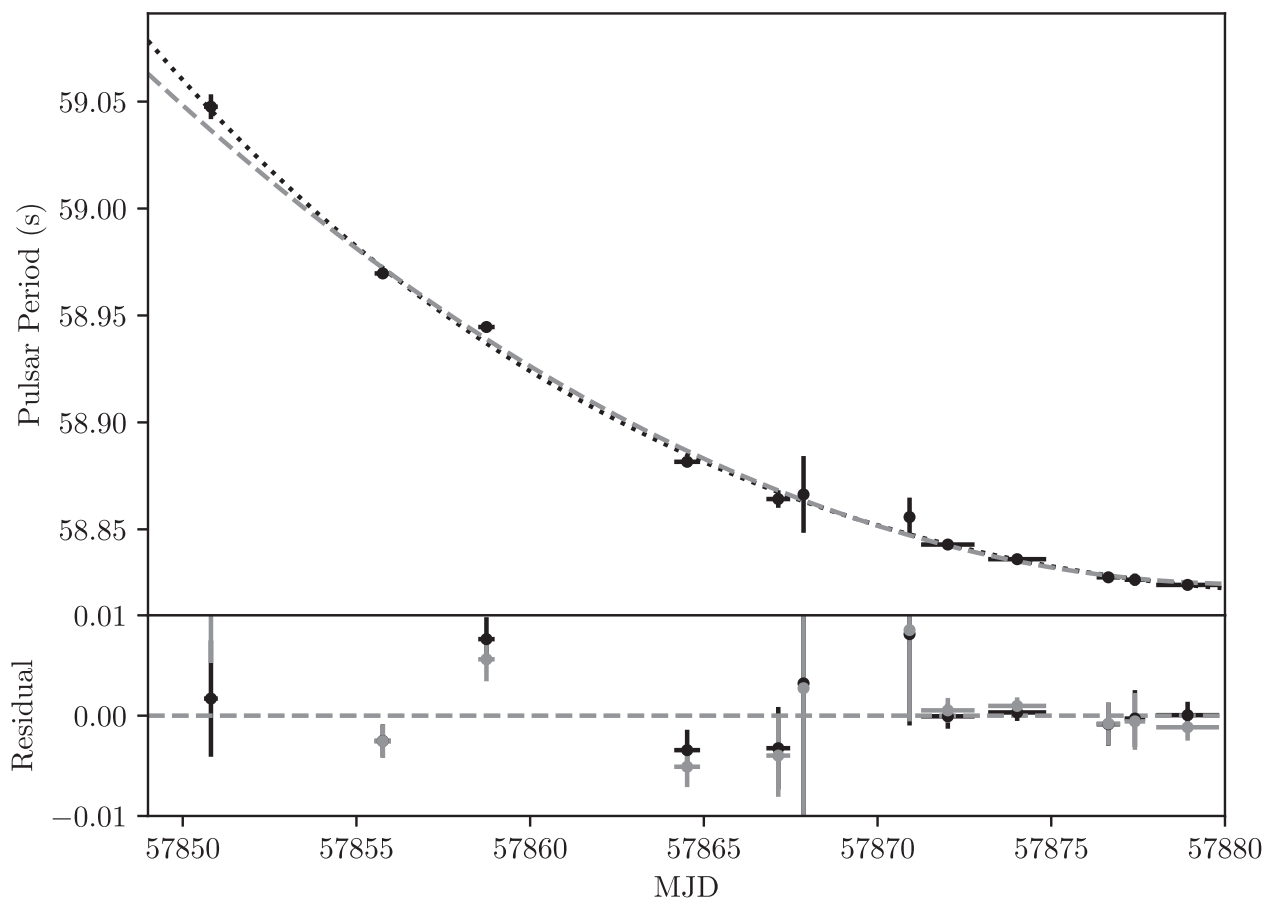

Figure 14. Pulsar period evolution of SXP 59.0 during its outburst in early 2017, utilizing WT data taken as part of a ToO campaign to study the outburst. Two models are fit to the data: a simple spin-up with $\dot{P}$ and $\ddot{P}$ components (black dotted line), and a Doppler-shifted pulsar spin at the orbital period of 122.25 days (gray dashed line).

$\tau \simeq 15.9$ days. Given the brightness and length of the outburst, this was likely a Type II burst.

In the Swift observation of 2017 April 7, a pulsar period of $P=59.0476 \pm 0.00165 \mathrm{~s}$ was detected. Measurements of the pulsar period are shown in Figure 14; after 2017 May 5, SXP 59.0 became too faint for an accurate period to be detected. The pulsar period during this time shows significant time variability. Modeling this as a simple spin-up, we obtain a $\dot{P}=$ $-(1.69 \pm 0.07) \times 10^{-7} \mathrm{~s} \mathrm{~s}^{-1}$ and $\ddot{P}=-(5.49 \pm 0.40) \times$ $10^{-9} \mathrm{~s} \mathrm{~s}^{-1} \mathrm{~s}^{-1}$. However, in these systems, pulsar spin period is 
often significantly affected by orbital-motion-induced Doppler shift, for example, as seen in Swift observations of SMC X-3 (Townsend et al. 2017) and SXP 5.05 (Coe et al. 2015), so we cannot rule out that orbital motion contributes to the observed pulsar period changes.

In order to estimate the probability of this, we fit a model consisting of a simple spin-up modified by Doppler-shifted orbital motion, similar to the method employed by Coe et al. (2015). As the orbital period is much longer than the $\sim 24$ days in which the pulsar period was detected, we fixed the orbital period to 122.25 days and fixed the orbital eccentricity to a typical value of $e=0.3$. The resultant fit is improved over the spin-up-only fit, reduced $\chi^{2}=2.13(9$ dof $)$ versus $\chi^{2}=2.57$ (9 dof) for the spin-up-only fit; however, derived orbital parameters are not well constrained. For the fit with orbital modulation, we derive an underlying spin-up $\dot{P}=-5.49 \times$ $10^{-9} \mathrm{~s} \mathrm{~s}^{-1}$, although given the uncertainties on the orbital modeling, we consider this value to have likely larger uncertainties than the quoted fit errors.

It is a clear that in order to derive an orbital solution for SXP 59.0 utilizing pulsar timing, a longer outburst in which the spin period of the pulsar was measurable for at least one full orbital period would be necessary. We note that an L-S search of the light curve of SXP 59.0 (see Section 5.5) did not find a significant period. SXP 59.0 does show possible evidence of a single Type I outburst; however, the S-CUBED light curve is dominated by the Type II outburst during the period when a second periastron passage would occur, making detection of the relatively long (122-day) orbital period not possible.

\subsubsection{SXP 6.85}

SXP 6.85 (SC49) is a BeXRB in the SMC, first found by RXTE and named XTE J0103-728 (Corbet et al. 2003b). It is associated with a Be-star companion based on localization by XMM-Newton during an outburst seen in 2006 October (Haberl et al. 2007) and is therefore a BeXRB. Analysis of OGLE data found a likely orbital period of $P_{\text {orb }} \simeq 24.8$ days (Schmidtke et al. 2015). A further outburst of this system was detected by INTEGRAL on 2015 April (Nikolajuk et al. 2015).

The S-CUBED light curve of SXP 6.85 is shown in Figure 10. Initial observations did not detect the source; however, starting on 2016 July 29, SXP 6.85 was significantly detected, with peak luminosities of $(1.6 \pm 0.4) \times 10^{37} \mathrm{erg} \mathrm{s}^{-1}$ $(0.5-10 \mathrm{keV}$ at $62 \mathrm{kpc})$. Examination of archival data at this location reveals that Swift observed SXP 6.85 serendipitously in PC mode for a total of $15.7 \mathrm{ks}$ between 2016 March 25 and 28, as it lay near the GRB 160325A (Sonbas et al. 2016). During that observation, no photons were detected from the location of SXP 6.85, allowing us to place strong upper limits on the X-ray count rate of the source approximately 4 months before its 2016 July 29 outburst of $<2.4 \times 10^{34} \mathrm{erg} \mathrm{s}^{-1}$. No follow-up observations were made to confirm the pulsar period; however, the S-CUBED localization is consistent with that reported by XMM-Newton.

After the initial outburst detection, SXP 6.85 appears to fade over a period of 50 days (see Figure 10); given the purported 24.8-day orbital period of this BeXRB, we conclude that this is a Type II outburst. Starting 2016 December 28, the source appears to undergo a second flare, peaking around 2017 February.

As reported in Table 4, SXP 6.85 displays a large discrepancy between the orbital period of $\sim 24.8$ days and the
S-CUBED-detected period of 161.6 days. Given the presence of two similar-level, likely Type II flares in the S-CUBED light curve, the origin of this detected period can be explained by it being the time between these two events. As only two full cycles at this period exist in the first year of S-CUBED data, more observations over a longer timescale are needed to confirm whether these outbursts are indeed periodic.

\subsubsection{SXP 2.16}

SXP 2.16 is a BeXRB, first discovered by RXTE (as XTE J0119-731; Corbet et al. 2003a). In 2014 a bright transient in the SMC, IGR J01217-7257, was identified in outburst by INTEGRAL; however, a follow-up Swift WT mode observation could not confirm the presence of pulsations to confirm that it was indeed SXP 2.16 (Coe et al. 2014). An XMM-Newton observation performed in 2015 detected the presence of the $2.16 \mathrm{~s}$ periodicity, confirming that IGR J01217 -7257 and XTE J0119-731 are the same source: SXP 2.16 (Vasilopoulos et al. 2017a).

Some results from the S-CUBED observations of SXP 2.16 have been previously reported by Boon et al. (2017). For the majority of the period of interest reported in this paper, SXP 2.16 was not detected. However, it underwent two short outbursts in which it was above the detection limit. SXP 2.16 was detected in three consecutive S-CUBED observations that took place on 2016 July 6, 10, and 15, as reported by Boon et al. (2017). Additional detections of SXP 2.16 were made by S-CUBED during observations taking place on 2016 September 28 and October 5. Boon et al. (2017) report estimates of the orbital period from BAT data and OGLE $I$-band light curves of $82.5 \pm 0.7$ days and $83.67 \pm 0.05$ days, respectively. The time between the onset of the two periods of detection in S-CUBED is 84 days, which is consistent with these outbursts recurring at the reported orbital period, suggesting that these are likely Type I outbursts.

The S-CUBED light curve of SXP 2.16 is shown in Figure 15, with gray lines representing estimates of the expected periastron passage times, assuming an orbital period of $P_{\text {orb }}=82.5$ days, and assuming a periastron passage epoch of 2016 June 21, based on the center of the first outburst seen by S-CUBED.

It should be noted that no other outbursts were seen, despite two more periastron passages occurring during the period of interest. Although the lengths of the detected outbursts are uncertain owing to the relatively infrequent sampling of S-CUBED, the time between the first and last detection of the first outburst was 9.2 days, and for the second outburst it was 7 days; we therefore suggest that 7 days should be considered the lower limit for the outburst timescale.

S-CUBED observations were performed on 2016 December 14 (10 days before), and the predicted periastron passage centered around 2016 December 24 and 28 (4 days after); given this, it is plausible that the expected Type I outburst was simply missed owing to the fact that S-CUBED observations were taken 15 days apart during this period.

For the final periastron passage, centered around 2017 March 17, observations by S-CUBED were taken on 2017 March 14 (3 days before) and 2017 March 22 (5 days later), an 8-day gap. It is clear that an outburst lasting $>10$ days would likely have been detected during this observation period. However, we cannot strongly rule out having missed a $<8$-day-long outburst. 


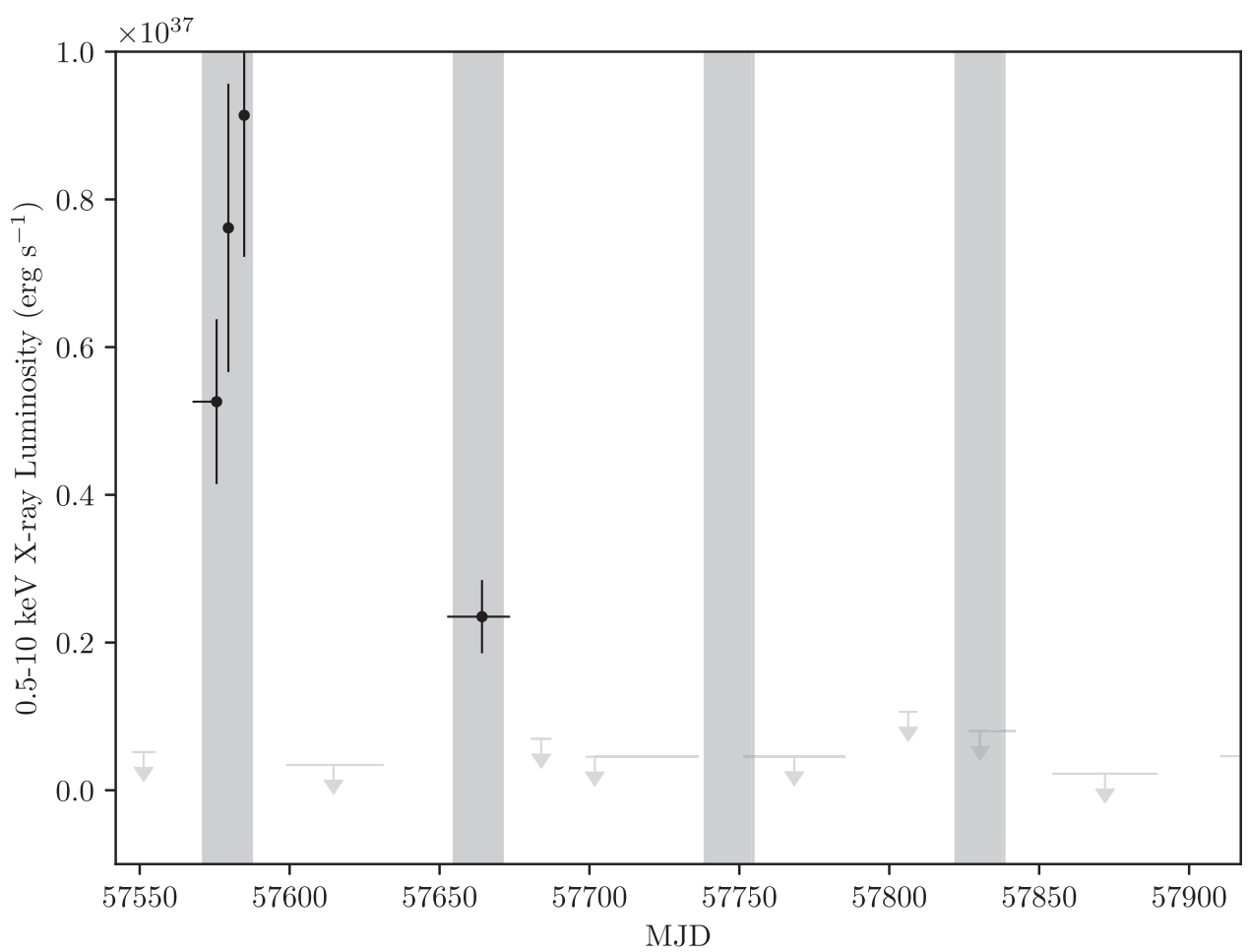

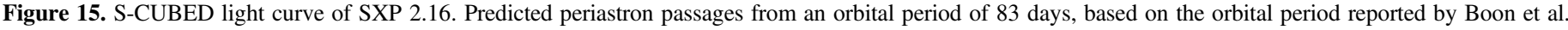

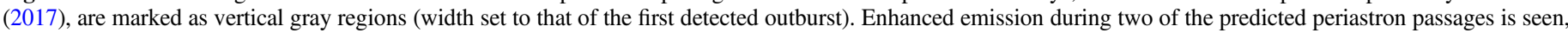

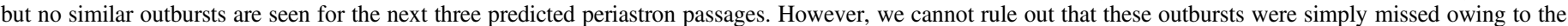
low sampling rate of S-CUBED.

During the reported period, no additional observations of SXP 2.16 were taken by Swift.

An L-S periodogram search of the S-CUBED light curve reveals a peak periodicity at $80.25 \pm 0.40$ days as reported in Table 4, which is somewhat inconsistent with the values reported by Boon et al. (2017). However, an L-S search of only the first $0.5 \mathrm{yr}$ of S-CUBED data, which contains the two outbursts, finds a peak period of $83.18 \pm 0.45$ days, consistent within errors with the BAT-derived orbital period, and close to the orbital period.

\subsubsection{SXP 15.6}

SXP 15.6 (SC3) was detected by S-CUBED in an observation taken on 2016 June 16, as an X-ray point source with a position consistent with the location of the cataloged X-ray source XMMU J004855.5-734946, reported to be a possible HMXB (Haberl \& Sturm 2016). In the following observation taken on 2016 June 24, it was found to have brightened, reaching a luminosity of $\sim 8 \times 10^{37} \mathrm{erg} \mathrm{cm}^{-2}$, significantly brighter than the cataloged XMM-Newton brightness of $6.4 \times 10^{-13} \mathrm{erg} \mathrm{cm}^{-2} \mathrm{~s}^{-1}$ (Evans et al. 2016a), which at $62 \mathrm{kpc}$ is a luminosity of $\sim 3 \times 10^{35} \mathrm{erg} \mathrm{s}^{-1}$. XMM-Newton observations revealed a $\sim 15.6 \mathrm{~s}$ period, strongly suggesting that the source is a BeXRB (Vasilopoulos et al. 2017b). SXP 15.6 was reported to have an optical counterpart in the catalog of Evans et al. (2004), with a spectral type of B0 IV-Ve, firmly confirming it as a BeXRB (McBride et al. 2017).

The S-CUBED light curve of SXP 15.6 shows brief flaring intervals (Figure 10) alongside periods of nondetection, which appear to be periodic. As reported in Table 4, SXP 15.6 is found to have a high significance period detection at $36.82 \pm$ 1.53 days (see Table 4). This periodicity is consistent with the orbital period derived from the OGLE IV light curve of $36.43 \pm 0.01$ days (McBride et al. 2017). We therefore suggest that the S-CUBED light curve shows a strong signature of repeated Type I outbursts at the orbital period.

\subsubsection{SXP $202 A$}

SXP 202A (SC20) is a BeXRB first detected in observations by XMM-Newton in 2003 (Majid et al. 2004). S-CUBED detected an apparent outburst of SXP 202A observations taken on 2016 July 6, 10, and 16 (Coe et al. 2016a). This outburst peaked at a luminosity of $(8.3 \pm 2.6) \times 10^{36} \mathrm{erg} \mathrm{s}^{-1}$ $(0.5-10 \mathrm{keV})$, consistent with the range seen for Type I BeXRB outbursts. Outside of this outburst SXP 202A is detected in $40 \%$ of S-CUBED observations at a mean luminosity of $(2.8 \pm 0.6) \times 10^{36} \mathrm{erg} \mathrm{s}^{-1}(0.5-10 \mathrm{keV})$, with upper limits calculated for the nondetections that are consistent with this emission level. No other outburst was detected during the first year of S-CUBED observations.

If the outburst seen by SXP 202A was in fact a Type I outburst, no recurrence was seen in the first year of observations, although we cannot strongly rule out that a Type I outburst did not occur during an observation gap. Currently SXP 202A does not have a measured orbital period, although based on the empirically measured relationship between pulsar spin and orbital period in BeXRBs (the "Corbet Diagram"; Corbet 1984), we would expect the orbital period to be long ( 200 days). The nondetection of repeating outbursts in SXP 202A does not allow us to place constraints on the orbital period in this system. Examination of the L-S periodogram does not show any significant peaks. Longer observations of SXP 202A will be required in order to pin down any orbital modulation. 


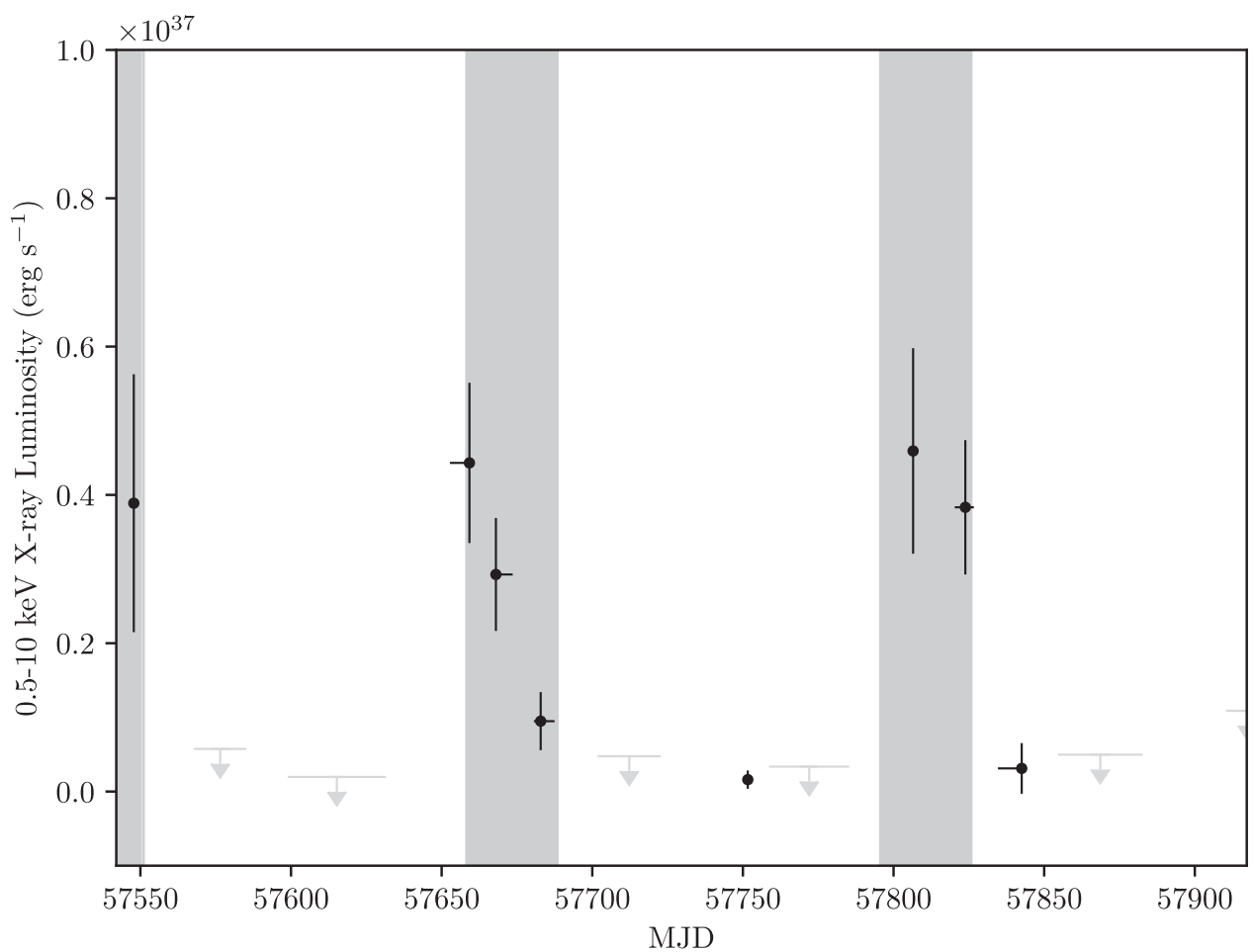

Figure 16. S-CUBED light curve of SXP 46.6 with predicted periastron passages marked in gray, based on the orbital period of 137.4 days, with the periastron passage fixed to the maximum flux point at 2016 October 19 (MJD 57,670). Three orbital periods are covered by the S-CUBED monitoring, and detections of SXP 46.6 during these periods are consistent with Type I outbursts.

\subsubsection{SXP 46.6}

SXP 46.6 (SC271) is a BeXRB (Coe \& Kirk 2015) with a reported orbital period of $P_{\text {orb }}=137.4 \pm 0.2$ days based on OGLE light curves (Bird et al. 2012) and a consistent X-rayderived $P_{\text {orb }}=137.36$ (Galache et al. 2008). As noted in Section 5.5, S-CUBED data show a significantly detected period of $143.29 \pm 4.5$ days, close to the previously reported orbital periods.

The S-CUBED light curve of SXP 46.6 (see Figure 16) reveals apparent low-level activity from SXP 46.6 during the year of observations, with detections of the source made during three periods, first during the first observation on 2016 June 8, second during an apparent small outburst, starting on 2016 September 28 and fading away with the last detection on 2016 October 19, and a final period with three detections between 2017 February 22 and March 14. Figure 16 shows the predicted period of time in which a Type I outburst would occur, assuming a 137.4-day orbital period, with the prominent outburst in the S-CUBED data as the assumed periastron passage time. It is clear that both the early initial detection and the later detections are consistent with being part of a Type I outburst.

\subsection{9. $S X P$ 91.1}

SXP 91.1 (SC6) is a BeXRB, which was first discovered by RXTE (named RX J0051.3-7216) with a $92 \pm 1.5 \mathrm{~s}$ pulsar period. Townsend et al. (2013) determined an orbital period measurement of $88.42 \pm 0.14$ days and showed that SXP 91.1 has a significant trend of long-term pulsar spin-up with $\dot{P}=(1.442 \pm 0.005) \times 10^{-8} \mathrm{~s} \mathrm{~s}^{-1}$. Further orbital period estimates from optical data were reported to be 88.2 days (Schmidtke \& Cowley 2006) and $88.37 \pm 0.03$ days (Bird et al. 2012).
The light curve of SXP 91.1 shown in Figure 10 shows periods of apparent enhanced X-ray emission and periods where the source was not active. We detect a periodicity in S-CUBED data of $P=89.25 \pm 0.03$ days, consistent with the previously reported orbital period. The false-alarm probability of this peak is found to be $\sim 2 \times 10^{-7}$, suggesting an unambiguous detection of the orbital period in the S-CUBED data.

S-CUBED monitoring shows two well-detected Type I outbursts centered on 2016 December 8 and 2017 March 6. Three other periastron passages show enhanced X-ray emission but are not as well sampled, and the peak emissions were likely missed.

\section{Discussion and Conclusions}

This paper presents results from the first year of the S-CUBED survey of the SMC. S-CUBED is focused on discovery of new X-ray outbursts by known and unknown $\mathrm{X}$-ray transients, which in the case of the SMC are mostly BeXRBs where the X-ray emission is due to Type I and Type II X-ray outbursts.

During the first year of S-CUBED observations, two major Type II outbursts of BeXRBs were detected in the SMC: SMC X-3 and SXP 59.0. In addition to these two bright outbursts, SXP 6.85 showed strong evidence of two extended outbursts, which are likely also Type II in nature. The 2016-2017 super-Eddington (peaking at $1.4 \times 10^{39} \mathrm{erg} \mathrm{s}^{-1}$ ) Type II outburst of SMC X-3, which was first identified by S-CUBED and has been reported on extensively in the literature (Townsend et al. 2017; Tsygankov et al. 2017; Weng et al. 2017), shows the power of the S-CUBED observing technique to catch bright outbursts early. S-CUBED triggered 
extensive follow-up campaigns, including Swift deeper observations, which were able to measure the spin evolution of the pulsar during the bright Type I outburst and model the orbital parameters.

The bright Type II outburst from SXP 59.0 also triggered follow-up observations with Swift. The peak flux of the outburst of SXP 59.0 was much fainter than the outburst of SMC X-3; however, this outburst was quickly identified by S-CUBED. Results from both S-CUBED and Swift ToO observations of SXP 59.0 are reported for the first time in this paper.

In addition to the bright Type II outbursts, direct evidence of Type I outbursts was seen from at least three known BeXRBs: SMC X-3 after the Type II outburst had ended; SXP 46.6, which was mostly not detected except for three outbursts that occurred at intervals consistent with the reported 137.4-day orbital period; and SXP 2.16, which showed two outbursts separated by $\sim 83$ days, close to the reported orbital period, but no more obvious outbursts after that.

We have performed period searches of the S-CUBED data in order to search for any possible orbital periods. S-CUBED detected emission from a total of 29 SXP sources for which the periods have been previously reported either from optical or X-ray measurements. Of those 29 sources, we positively detect an orbital period for 8 of them (see Table 4).

Observing the bulk properties of sources in the S-CUBED survey, we measured the DC and note that the DC plot in Figure 7 does show evidence that the maximum value of DC decreases with orbital period. This raises the question, are we missing Type I outbursts? In many cases it can be explained that S-CUBED is simply missing them owing to the nonuniform nature of the coverage. However, the most likely scenario is that the outbursts are below the level of detection for these short exposures. For example, S-CUBED monitoring has shown that in the case of objects like SXP 2.16 the flux level of Type I outbursts varies. Reig (2011) has shown examples of transient and nontransient behaviors of Type I bursts in BeXRBs: EXO 2030+375, which shows regular Type I bursts, and $4 \mathrm{U} 0115+63$, which shows only occasional detections of Type I bursts. Given this, the wide scatter in the measured DC and the presence of an apparent maximum DC that decreases as orbital period gets larger are the expected behavior.

Results from the first year of S-CUBED presented here show both the strengths and weaknesses of the survey. For detection of Type I outbursts, comparing the results from the postoutburst ToO observations of SMC X-3 to S-CUBED-only data, it would clearly be beneficial to observe both more frequently and with a longer exposure in order to better detect these events. However, due to limitations in the amount of available observing time, it is not likely that this could be achieved with Swift without significantly changing the design of the survey. However, where periods were detected in the S-CUBED survey, they have very good agreement with those previously reported in the literature. Out of 265 S-CUBED detected "Good" X-ray sources in the vicinity of the SMC during the first year of the survey, the majority (160) are previously uncataloged. Many of these sources are in regions previously surveyed to much higher sensitivities by Chandra, $X M M-N e w t o n$, and others. We believe that these sources are real to high confidence and represent the discovery of a large population of variable sources, which could have only been discovered by performing repeated searches, rather than a single deep survey. This result strongly validates the approach of S-CUBED.

J.A.K. acknowledges the support of NASA contract NAS500136 and NASA grant NNX16AR15G (through the Swift Guest Investigator program). This work made use of data supplied by the UK Swift Science Data Centre at the University of Leicester. The Digitized Sky Surveys were produced at the Space Telescope Science Institute under U.S. Government grant NAG W-2166. The images of these surveys are based on photographic data obtained using the Oschin Schmidt Telescope on Palomar Mountain and the UK Schmidt Telescope. The plates were processed into the present compressed digital form with the permission of these institutions.

\section{ORCID iDs}

J. A. Kennea (iD https://orcid.org/0000-0002-6745-4790

M. J. Coe (iD https://orcid.org/0000-0002-0763-8547

P. A. Evans (ii) https://orcid.org/0000-0002-8465-3353

\section{References}

Antoniou, V., Zezas, A., Hatzidimitriou, D., \& McDowell, J. C. 2009, ApJ, 697, 1695

Astropy Collaboration, Robitaille, T. P., Tollerud, E. J., et al. 2013, A\&A, 558, A33

Azzopardi, M., \& Vigneau, J. 1979, A\&AS, 35, 353

Barthelmy, S. D., Barbier, L. M., Cummings, J. R., et al. 2005, SSRv, 120, 143 Bird, A. J., Coe, M. J., McBride, V. A., \& Udalski, A. 2012, MNRAS, 423, 3663

Boon, C. M., Bird, A. J., Coe, M. J., et al. 2017, MNRAS, 466, 1149

Bradt, H. V., Rothschild, R. E., \& Swank, J. H. 1993, A\&AS, 97, 355

Burrows, D. N., Hill, J. E., Nousek, J. A., et al. 2005, SSRv, 120, 165

Carpano, S., Haberl, F., \& Sturm, R. 2017, A\&A, 602, A81

Casares, J., Negueruela, I., Ribó, M., et al. 2014, Natur, 505, 378

Cioni, M. R. L., Kamath, D., Rubele, S., et al. 2013, A\&A, 549, A29

Clark, G., Doxsey, R., Li, F., Jernigan, J. G., \& van Paradijs, J. 1978, ApJL, 221, L37

Coe, M. J., Bartlett, E. S., Bird, A. J., et al. 2015, MNRAS, 447, 2387

Coe, M. J., Bird, A. J., Buckley, D. A. H., et al. 2010, MNRAS, 406, 2533

Coe, M. J., Bird, A. J., McBride, V. A., et al. 2014, ATel, 5806

Coe, M. J., Edge, W. R. T., Galache, J. L., \& McBride, V. A. 2005, MNRAS, 356, 502

Coe, M. J., Evans, P. A., Kennea, J. A., \& Udalski, A. 2016a, ATel, 9307

Coe, M. J., Kennea, J. A., Evans, P. A., McHardy, I., \& Udalski, A. 2016b, ATel, 9414

Coe, M. J., Kennea, J. A., Townsend, L. J., et al. 2016c, ATel, 9677

Coe, M. J., \& Kirk, J. 2015, MNRAS, 452, 969

Corbet, R., Markwardt, C. B., Marshall, F. E., et al. 2003a, IAUC, 8064

Corbet, R. H. D. 1984, A\&A, 141, 91

Corbet, R. H. D., Markwardt, C. B., Marshall, F. E., et al. 2003b, ATel, 163

Cutri, R. M., Skrutskie, M. F., van Dyk, S., et al. 2003, yCat, 2246

Edge, W. R. T., Coe, M. J., Galache, J. L., et al. 2004, MNRAS, 353, 1286

Evans, C. J., Howarth, I. D., Irwin, M. J., Burnley, A. W., \& Harries, T. J. 2004, MNRAS, 353, 601

Evans, P. A., Beardmore, A. P., Page, K. L., et al. 2007, A\&A, 469, 379

Evans, P. A., Beardmore, A. P., Page, K. L., et al. 2009, MNRAS, 397, 1177

Evans, P. A., Kennea, J. A., \& Coe, M. J. 2016a, ATel, 9197

Evans, P. A., Osborne, J. P., Beardmore, A. P., et al. 2014, ApJS, 210, 8

Evans, P. A., Osborne, J. P., Kennea, J. A., et al. 2016b, MNRAS, 455, 1522

Galache, J. L., Corbet, R. H. D., Coe, M. J., et al. 2008, ApJS, 177, 189

Gehrels, N., Chincarini, G., Giommi, P., et al. 2004, ApJ, 611, 1005

Goad, M. R., Tyler, L. G., Beardmore, A. P., et al. 2007, A\&A, 476, 1401

Gotthelf, E. V., Vasisht, G., \& Dotani, T. 1999, ApJL, 522, L49

Grimm, H.-J., Gilfanov, M., \& Sunyaev, R. 2003, MNRAS, 339, 793

Haberl, F., Filipović, M. D., Pietsch, W., \& Kahabka, P. 2000, A\&AS, 142, 41

Haberl, F., Pietsch, W., \& Kahabka, P. 2007, ATel, 1095

Haberl, F., \& Sturm, R. 2016, A\&A, 586, A81

Harris, J., \& Zaritsky, D. 2004, AJ, 127, 1531

Haschke, R., Grebel, E. K., \& Duffau, S. 2012, AJ, 144, 107 
Hong, J., Antoniou, V., Zezas, A., et al. 2017, ApJ, 847, 26

Kahabka, P., \& Pietsch, W. 1996, A\&A, 312, 919

Kato, D., Nagashima, C., Nagayama, T., et al. 2007, PASJ, 59, 615

Kennea, J. A., Coe, M. J., Evans, P. A., et al. 2016a, ATel, 9362

Kennea, J. A., Evans, P. A., \& Coe, M. J. 2016b, ATel, 9299

Kennea, J. A., Evans, P. A., \& Coe, M. J. 2017, ATel, 10250

Klus, H., Ho, W. C. G., Coe, M. J., Corbet, R. H. D., \& Townsend, L. J. 2014, MNRAS, 437, 3863

Koliopanos, F., \& Vasilopoulos, G. 2018, A\&A, 614, A23

Kozłowski, S., Kochanek, C. S., Jacyszyn, A. M., et al. 2012, ApJ, 746, 27

Kozłowski, S., Kochanek, C. S., \& Udalski, A. 2011, ApJS, 194, 22

Kozłowski, S., Onken, C. A., Kochanek, C. S., et al. 2013, ApJ, 775, 92

Krimm, H. A., Holland, S. T., Corbet, R. H. D., et al. 2013, ApJS, 209, 14

Li, F., Jernigan, G., \& Clark, G. 1977, IAUC, 3125

Li, X.-D., \& van den Heuvel, E. P. J. 1997, A\&A, 321, L25

Lomb, N. R. 1976, Ap\&SS, 39, 447

Majid, W. A., Lamb, R. C., \& Macomb, D. J. 2004, ApJ, 609, 133

Marshall, F. E., Lochner, J. C., Santangelo, A., et al. 1998, IAUC, 6818

Massey, P. 2002, ApJS, 141, 81

Matsuoka, M., Kawasaki, K., Ueno, S., et al. 2009, PASJ, 61, 999

McBride, V. A., González-Galán, A., Bird, A. J., et al. 2017, MNRAS, 467, 1526

McGowan, K. E., Coe, M. J., Schurch, M. P. E., et al. 2008, MNRAS, 383, 330

Meegan, C., Lichti, G., Bhat, P. N., et al. 2009, ApJ, 702, 791

Meyssonnier, N., \& Azzopardi, M. 1993, A\&AS, 102, 451

Negoro, H., Nakajima, M., Kawai, N., et al. 2016, ATel, 9348

Nikolajuk, M., Bozzo, E., \& Ferrigno, C. 2015, ATel, 7481

Plucinsky, P. P., Beardmore, A. P., Foster, A., et al. 2017, A\&A, 597, A35

Reig, P. 2011, Ap\&SS, 332, 1

Roming, P. W. A., Kennedy, T. E., Mason, K. O., et al. 2005, SSRv, 120, 95
Rosen, S. R., Webb, N. A., Watson, M. G., et al. 2016, A\&A, 590, A1

Scargle, J. D. 1982, ApJ, 263, 835

Schmidtke, P. C., \& Cowley, A. P. 2006, AJ, 132, 919

Schmidtke, P. C., Cowley, A. P., \& Udalski, A. 2015, ATel, 7498

Schurch, M. P. E., Coe, M. J., McBride, V. A., et al. 2011, MNRAS, 412, 391

Schurch, M. P. E., Coe, M. J., McGowan, K. E., et al. 2007, MNRAS, 381,1561

Secrest, N. J., Dudik, R. P., Dorland, B. N., et al. 2015, ApJS, 221, 12

Shtykovskiy, P., \& Gilfanov, M. 2005, MNRAS, 362, 879

Sonbas, E., Lien, A. Y., \& Page, K. L. 2016, GCN, 1, 19222

Stella, L., White, N. E., \& Rosner, R. 1986, ApJ, 308, 669

Sturm, R., Haberl, F., Pietsch, W., et al. 2013, A\&A, 558, A3

Townsend, L. J., Drave, S. P., Hill, A. B., et al. 2013, MNRAS, 433, 23

Townsend, L. J., Kennea, J. A., Coe, M. J., et al. 2017, MNRAS, 471, 3878

Trowbridge, S., Nowak, M. A., \& Wilms, J. 2007, ApJ, 670, 624

Tsygankov, S. S., Doroshenko, V., Lutovinov, A. A., Mushtukov, A. A., \& Poutanen, J. 2017, A\&A, 605, A39

Udalski, A., Kubiak, M., \& Szymanski, M. 1997, AcA, 47, 319

Udalski, A., Szymański, M. K., \& Szymański, G. 2015, AcA, 65, 1

VanderPlas, J. T. 2018, ApJS, 236, 16

Vasilopoulos, G., Haberl, F., \& Maggi, P. 2017a, MNRAS, 470, 1971

Vasilopoulos, G., Zezas, A., Antoniou, V., \& Haberl, F. 2017b, MNRAS, 470, 4354

Véron-Cetty, M.-P., \& Véron, P. 2010, A\&A, 518, A10

Voges, W., Aschenbach, B., Boller, T., et al. 1999, A\&A, 349, 389

Weng, S.-S., Ge, M.-Y., Zhao, H.-H., et al. 2017, ApJ, 843, 69

Willingale, R., Starling, R. L. C., Beardmore, A. P., Tanvir, N. R., \& O'Brien, P. T. 2013, MNRAS, 431, 394

Yang, J., Laycock, S. G. T., Christodoulou, D. M., et al. 2017, ApJ, 839, 119 